\title{
EMBEDDINGS OF SPHERICAL HOMOGENEOUS SPACES
}

\author{
JACOPO GANDINI
}

\begin{abstract}
We review in these notes the theory of equivariant embeddings of spherical homogeneous spaces. Given a spherical homogeneous space $G / H$, the normal equivariant embeddings of $G / H$ are classified by combinatorial objects called colored fans, which generalize the fans appearing in the classification of toric varieties and which encode several geometric properties of the corresponding variety.
\end{abstract}

\section{Contents}

1. Introduction

2. Characterizations of sphericality 3

3. Examples 9

4. Invariant valuations on a spherical homogeneous space 12

5. The $B$-stable affine open subset associated to a $G$-orbit 15

6. Simple spherical embeddings and colored cones 17

7. The classification of spherical embeddings 23

8. Morphisms between spherical embeddings 24

9. Co-connected inclusions and colored subspaces 28

10. The valuation cone of a spherical homogeneous space 30

11. Equivariant automorphisms of a spherical homogeneous space 32

12. Wonderful embeddings and spherical roots 36

References $\quad 38$

\section{INTRODUCTION}

These notes are based on a series of lectures that I gave at the workshop Spherical varieties, organized by Michel Brion and Baohua Fu at the Tsinghua Sanya International Mathematics Forum in November 2016.

Given a connected reductive group $G$ over an algebraically closed field of characteristic zero, a general theory of the embeddings of a homogeneous space $G / H$ was formulated by Luna and Vust [24]. An invariant of $G / H$ which plays an important role here is the complexity of $G / H$, that is, the minimal codimension of a $B$-orbit in $G / H$, where $B$ is a fixed Borel subgroup of $G$. The theory of Luna and Vust works particularly well when the complexity of $G / H$ is zero, in which case $G / H$ is also called spherical.

The class of spherical homogeneous spaces contains several important families which were studied independently and whose embedding theories are interesting in their own right. For example, torus embeddings, embeddings of symmetric varieties (i.e. $H$ is the set of fixed points of an algebraic involution of $G$ ), embeddings of horospherical varieties (i.e. $H$ contains a maximal unipotent subgroup of $G$ ) all fall within the theory of spherical embeddings.

The theory of spherical embeddings was then extended to arbitary characteristics by Knop [17]. Other expositions can be found in Timashev's book [34], where the 
case of complexity 1 is also considered, in Brion's lecture notes [5] and in Pezzini's lecture notes [27] (the latter two in characteristic zero).

Both in the exposition of the material and in the arguments that we present, we mainly followed Knop's paper [17]. In order to make the material as accessible as possible for those who are new to the subject, detailed arguments are given, as well as some examples treated in detail. A basic knowledge of algebraic geometry and of algebraic groups is assumed, for which we refer respectively to [12] and to [31].

Acknowledgements. I am grateful to Michel Brion and Baohua Fu, both for the invitation and for organizing this workshop and the conference which followed it. I thank Johannes Hofscheier and Dmitry Timashev for helpful discussions, and especially Bart Van Steirteghem and the referee for several remarks and suggestions which improved these notes.

Notation and conventions on algebraic groups and varieties. Throughout the paper, we fix an algebraically closed field $\mathrm{k}$ of characteristic $p \geqslant 0$. Every variety will be defined over $k$, and is assumed to be irreducible. We will denote by $\mathbb{G}_{m}$ and by $\mathbb{G}_{a}$ resp. the multiplicative group and the additive group of $\mathrm{k}$, regarded as algebraic groups. Given an algebraic group $K$, we denote by $K^{0}$ its connected component containing the identity, by $K_{\mathrm{u}}$ its unipotent radical and by $\mathcal{X}(K)$ its group of characters. If $V$ is a $K$-module, we denote by $V^{K}$ the subspace of $K$ invariants and by $V^{(K)}$ the subspace of $K$-semi-invariants. If moreover $\chi \in \mathcal{X}(K)$, we denote

$$
V_{\chi}^{(K)}=\{v \in V \mid k . v=\chi(k) v \text { for all } k \in K\} .
$$

Throughout the paper, $G$ will denote a connected reductive group. We fix a Borel subgroup $B \subset G$ and a maximal torus $T \subset B$. The opposite Borel subgroup of $B$ is denoted by $B^{-}$, this is the unique Borel subgroup of $G$ such that $B \cap B^{-}=T$. The unipotent radicals of $B$ and $B^{-}$are respectively denoted by $U$ and $U^{-}$. We denote by $\mathcal{X}(T)^{+} \subset \mathcal{X}(T)$ the monoid of dominant characters with respect to $B$. Finally, we denote by $W$ be the Weyl group of $G$ with respect to $T$, and by $w_{0} \in W$ the longest element defined by $B$.

If $H \subset G$ is a closed subgroup and $\chi \in \mathcal{X}(H)$, let

$$
\operatorname{Ind}_{H}^{G}(\chi)=\left\{f \in \mathrm{k}[G] \mid f(g h)=\chi\left(h^{-1}\right) f(g) \quad \forall g \in G, \forall h \in H\right\}
$$

be the corresponding induced representation of $G$ (see [15, Chapter I.3]). If char $(\mathrm{k})=$ 0 and $\lambda \in \mathcal{X}(T)^{+}$, we denote by $V(\lambda)$ the simple $G$-module of highest weight $\lambda$ and by $V^{*}(\lambda)$ its dual. More generally, in arbitrary characteristic, we denote by $V(\lambda)$ the Weyl module of highest weight $\lambda$, and by $V^{*}(\lambda)$ its dual, namely $V^{*}(\lambda)=\operatorname{Ind}_{B}^{G}(-\lambda)$ (see [15, Chapter II.2]).

If $V$ is a $G$-module and $\lambda \in \mathcal{X}(T)^{+}$, we define the multiplicity of $V(\lambda)$ in $V$ by

$$
m_{\lambda}(V):=\operatorname{dim}_{\mathrm{k}} \operatorname{Hom}_{G}(V(\lambda), V) .
$$

By Frobenius reciprocity, we have $m_{\lambda}(V)=\operatorname{dim} V_{\lambda}^{(B)}$. When $\operatorname{char}(\mathrm{k})=0$, this is the number of times that $V(\lambda)$ occurs in a decomposition of $V$ into simple $G$ modules.

A line bundle $p: \mathcal{L} \rightarrow X$ on a $G$-variety $X$ is said to be $G$-linearized if its total space is endowed with an action of $G$ such that $p$ is $G$-equivariant and the induced map on the fibers $\mathcal{L}_{x} \rightarrow \mathcal{L}_{g . x}$ is linear for all $g \in G$ and $x \in X$. If $\mathcal{L}$ is a $G$-linearized line bundle on $X$, then the space of sections $\Gamma(X, \mathcal{L})$ has a natural $G$-module structure, and all the powers $\mathcal{L}^{\otimes n}$ are $G$-linearized as well.

Remark 1.1. Let $H \subset G$ be a closed subgroup. The $G$-linearized line bundles on $G / H$ are easily described in terms of characters and associated bundles. If 
$\chi \in \mathcal{X}(H)$, let $\mathcal{L}_{\chi}=G \times{ }^{H} \mathrm{k}=(G \times \mathrm{k}) / / H$ be the categorical quotient of $G \times \mathrm{k}$ by the action of $H$ given by $h .(g, x)=\left(g h^{-1}, \chi(h) x\right)$ : such a quotient always exists, and it is also a geometric quotient (see [34, Section 2.1] for more details on associated bundles). Then $\mathcal{L}_{\chi}$ is a $G$-linearized line bundle on $G / H$, and the map $\chi \mapsto \mathcal{L}_{\chi}$ defines a homomorphism $\mathcal{X}(H) \rightarrow \operatorname{Pic}(G / H)$ whose image is the group of $G$-linearizable line bundles. Indeed if $p: \mathcal{L} \rightarrow G / H$ is a $G$-linearized line bundle and if $\chi \in \mathcal{X}(H)$ is the character given by the action of $H$ on the fiber $\mathcal{L}_{e H}$, then the natural morphism $G \times \mathcal{L}_{e H} \rightarrow \mathcal{L}$ induces a $G$-isomorphism $\mathcal{L} \simeq \mathcal{L}_{\chi}$. If $\operatorname{Pic}(G)=0$, then every line bundle on $G / H$ is $G$-linearizable, and if moreover $G$ is semisimple it can be linearized in a unique way: indeed we have an exact sequence

$$
\mathcal{X}(G) \rightarrow \mathcal{X}(H) \rightarrow \operatorname{Pic}(G / H) \rightarrow \operatorname{Pic}(G)
$$

(see [20, Proposition 4.6]). By the definition of induced representation, we have the following isomorphism of $G$-modules, with respect to the right action of $H$ on $\mathrm{k}[G]$ :

$$
\Gamma\left(G / H, \mathcal{L}_{\chi}\right) \simeq \operatorname{Ind}_{H}^{G}(\chi)=\mathrm{k}[G]_{-\chi}^{(H)} .
$$

Notations and conventions in convex geometry. Given a lattice $\Lambda$ we denote by $\Lambda^{\vee}=\operatorname{Hom}_{\mathbb{Z}}(\Lambda, \mathbb{Z})$ the dual lattice, and we set $\Lambda_{\mathbb{Q}}=\Lambda \otimes_{\mathbb{Z}} \mathbb{Q}$. A subset $\mathcal{C} \subset \Lambda_{\mathbb{Q}}$ is called a cone if it is closed under addition and multiplication by scalars in $\mathbb{Q}^{+}=$ $\{q \in \mathbb{Q} \mid q \geqslant 0\}$. The dual of $\mathcal{C}$ is the cone

$$
\mathcal{C}^{\vee}=\left\{\alpha \in \Lambda_{\mathbb{Q}}^{\vee} \mid\langle\alpha, v\rangle \geqslant 0 \quad \forall v \in \mathcal{C}\right\},
$$

and the annihilator of $\mathcal{C}$ is $\mathcal{C}^{\perp}=\mathcal{C}^{\vee} \cap\left(-\mathcal{C}^{\vee}\right)$. The linear part of $\mathcal{C}$ is the maximal subspace $\operatorname{Lin}(\mathcal{C}) \subset V$ which is contained in $\mathcal{C}$. If $v_{1}, \ldots, v_{n} \in \Lambda_{\mathbb{Q}}$, we denote by cone $\left(v_{1}, \ldots, v_{n}\right)$ the cone that they generate in $\Lambda_{\mathbb{Q}}$. A cone $\mathcal{C}$ is called strictly convex if $\operatorname{Lin}(\mathcal{C})=0$. It is called rational if it is generated by elements of $\Lambda$, finitely generated (or polyhedral) if it is generated by finitely many elements $v_{1}, \ldots, v_{n} \in$ $\Lambda_{\mathbb{Q}}$, and simplicial if it is generated by linearly independent vectors. A face of $\mathcal{C}$ is the intersection of $\mathcal{C}$ with a hyperplane $\alpha^{\perp}=\{v \in \mathcal{C} \mid\langle\alpha, v\rangle=0\}$ where $\alpha \in \mathcal{C}^{\vee}$. The dimension of $\mathcal{C}$ is the dimension of its linear span, an extremal ray is a face of dimension 1 . The relative interior of $\mathcal{C}$ is the subset $\mathcal{C}^{\circ}$ obtained by removing from $\mathcal{C}$ all its proper faces.

\section{Characterizations of SPhericality}

Definition 2.1. A normal $G$-variety is called spherical if it contains an open $B$ orbit.

Notice that every spherical variety $X$ possesses in particular an open $G$-orbit, which is a spherical homogeneous space. Therefore, once a base point $x_{0} \in X$ is fixed, we can regard $X$ as a normal equivariant embedding of a spherical homogeneous space. If moreover we assume that the orbit morphism $G \rightarrow G x_{0}$ is separable and if $H$ is the stabilizer of $x_{0}$, then we get an embedding of $G / H$.

By an embedding of a spherical homogeneous space $G / H$ we will always mean a $G$-variety $X$ together with an equivariant open embedding $G / H \hookrightarrow X$. We will say that $G / H \hookrightarrow X$ is a spherical embedding if moreover $X$ is normal. In particular, given a spherical embedding $G / H \hookrightarrow X$, we will identify the orbit morphism $G \rightarrow G x_{0}$ with the projection $\pi: G \rightarrow G / H$. We will say that a subgroup $H \subset G$ is spherical if $G / H$ is spherical. Unless otherwise stated, we will always assume that the base point $x_{0}$ is inside the open $B$-orbit, that is $B H$ is an open subset of $G$.

Example 2.2. We list here below some of the main examples of spherical varieties. 
i) (Flag varieties) Any complete homogeneous space is spherical. Indeed, it is of the form $G / P$ with $P$ a parabolic subgroup, hence it contains finitely many $B$-orbits by the Bruhat decomposition.

ii) (Horospherical varieties) Any homogeneous space $G / H$ with $H$ a closed subgroup containing $U$. Indeed, again by the Bruhat decomposition, $G / B$ contains an open $U$-orbit, hence an open $H$-orbit.

iii) (Toric varieties) If $G=T$ is a torus and $X$ is a normal $T$-equivariant embedding of $T$, then it is spherical with respect to $T$ (in which case $B=T$ ).

iv) (Reductive groups) Any reductive group $G$ is spherical with respect to the action of $G \times G$ by left and right multiplication. Indeed, by the Bruhat decomposition $B \times B$ has an open orbit on $G$.

v) (Symmetric matrices) Suppose that $\operatorname{char}(\mathrm{k}) \neq 2$ and let $X=\mathrm{Sym}_{n}$ be the space of symmetric matrices of order $n$, with the action of $\mathrm{GL}_{n}$ by congruence: for $g \in \mathrm{GL}_{n}$, we set $g \cdot A=\left(g^{-1}\right)^{T} A g^{-1}$. Let $B \subset \mathrm{GL}_{n}$ be the subgroup of upper triangular matrices and let $x_{n} \in X$ be the identity matrix: then $B x_{n} \subset X$ is open, hence $X$ is a spherical variety. Notice that the open $\mathrm{GL}_{n}$-orbit, isomorphic to $\mathrm{GL}_{n} / \mathrm{O}_{n}$, is the space of nonsingular symmetric matrices.

vi) (Determinantal varieties) Let $M_{m, n}$ be the space of $m \times n$-matrices, and let $M_{m, n, r} \subset M_{m, n}$ be the subset of matrices whose rank is at most $r$, endowed with the action of $\mathrm{GL}_{m} \times \mathrm{GL}_{n}$. Then the matrices of rank $r$ form an open $\left(\mathrm{GL}_{m} \times \mathrm{GL}_{n}\right)$-orbit. If

$$
x_{0}=\left(\begin{array}{cc}
I_{r} & 0 \\
0 & 0
\end{array}\right)
$$

then the stabilizer of $x_{0}$ consists of the matrices of the form

$$
\left(\begin{array}{ll}
A_{r, r} & B_{r, m-r} \\
0 & C_{m-r, m-r}
\end{array}\right) \times\left(\begin{array}{ll}
A_{r, r} & 0 \\
D_{n-r, r} & E_{n-r, n-r}
\end{array}\right) .
$$

Hence $M_{m, n, r}$ is an affine variety of dimension $r(m+n-r)$. Let $B_{m}^{-}$(resp. $B_{n}$ ) be the Borel subgroup of lower triangular (resp. upper triangular) matrices in $\mathrm{GL}_{m}$ (resp. in $\mathrm{GL}_{n}$ ). By computing dimensions it follows that the $\left(B_{m}^{-} \times B_{n}\right)$-orbit of $x_{0}$ is open in $M_{m, n, r}$. Since it is normal (see Example $3.5)$, it follows that $M_{m, n, r}$ is an affine spherical variety.

vii) (Symmetric spaces) Suppose that $\operatorname{char}(\mathrm{k}) \neq 2$ and let $\sigma: G \rightarrow G$ be an algebraic involution, let $G^{\sigma}$ be the subgroup of fixed points and let $H$ be a closed subgroup of $G$ such that $G^{\sigma} \subset H \subset \mathrm{N}_{G}\left(G^{\sigma}\right)$, then $G / H$ is called a symmetric space. As a consequence of the Iwasawa decomposition (see [34, Theorem 26.14]), every symmetric space is spherical. Example iv) is a particular instance of this situation, whereas Example v) is as an embedding of a symmetric space: in the first case, consider the involution of $G \times G$ defined by $\sigma\left(g_{1}, g_{2}\right)=\left(g_{2}, g_{1}\right)$, in the second case consider the involution of $\mathrm{GL}_{n}$ defined by $\sigma(g)=\left(g^{T}\right)^{-1}$.

We start by recalling some basic results from the theory of algebraic group and their invariant theory. We start with a very basic result, for an (easy) proof see [29, Lemma 1.4].

Lemma 2.3. If $K$ is a linear algebraic group acting on an algebraic variety $X$, then any element in $\mathrm{k}[X]$ is contained in a finite dimensional $K$-stable subspace.

The following is a fundamental result on the invariants of the maximal unipotent subgroup $U \subset G$, which will be essential for our purposes.

Theorem 2.4 ([13, Theorem 9.4]). Let $X$ be an affine $G$-variety, then $\mathrm{k}[X]^{U}$ is a finitely generated k-algebra. 
We now recall an important consequence of the geometric reductivity of $G$ (recall that reductive is equivalent to geometrically reductive, and if $\operatorname{char}(\mathrm{k})=0$ it is even equivalent to linearly reductive, see e.g. [9, Chapter 9]).

Theorem 2.5 ([13, Theorem 9.9]). Let $X$ be an affine $G$-variety, let $Y \subset X$ be closed and $G$-stable and let $f \in \mathrm{k}[Y]^{U}$. Then there exist $n \in \mathbb{N}$ and $f^{\prime} \in \mathrm{k}[X]^{U}$ such that $f_{\mid Y}^{\prime}=f^{n}$.

Notice that in the previous theorem one can always take $n=1$ if $\operatorname{char}(\mathrm{k})=0$, and a power of the characteristic if $\operatorname{char}(\mathrm{k})>0$. We can rephrase the previous theorem in terms of $B$-eigenfunctions as follows. Recall that $B=T U$, and since $U$ has no nontrivial characters we have $\mathrm{k}[X]^{(B)} \subset \mathrm{k}[X]^{U}$. On the other hand $T$ is linearly reductive, and since it normalizes $U$ it acts on $\mathrm{k}[X]^{U}$ : therefore

$$
\mathrm{k}[X]^{U}=\bigoplus_{\lambda \in \mathcal{X}(T)}\left(\mathrm{k}[X]^{U}\right)_{\lambda}^{(T)}=\bigoplus_{\lambda \in \mathcal{X}(B)} \mathrm{k}[X]_{\lambda}^{(B)} .
$$

Therefore taking into account the $T$-action as well we can restate Theorem 2.5 as follows.

Corollary 2.6. Let $X$ be an affine variety $G$-variety, let $Y \subset X$ be closed and $G$-stable and let $f \in \mathrm{k}[Y]^{(B)}$. Then there exist $n \in \mathbb{N}$ and $f^{\prime} \in \mathrm{k}[X]^{(B)}$ such that $f_{\mid Y}^{\prime}=f^{n}$.

We now give a classical characterization of sphericality.

Definition 2.7. We say that a $G$-module $V$ is multiplicity-free if $m_{\lambda}(V) \leqslant 1$ for all $\lambda \in \mathcal{X}(T)^{+}$.

Theorem 2.8. Let $X$ be a normal $G$-variety, then the following are equivalent:

i) $X$ is spherical;

ii) Any B-invariant rational function on $X$ is constant;

iii) If $X$ is quasi-projective: for every $G$-linearized line bundle $\mathcal{L}$ on $X$, the space of global sections $\Gamma(X, \mathcal{L})$ is a multiplicity-free $G$-module;

iii') If $X$ is quasi-affine: the coordinate ring $\mathrm{k}[X]$ is a multiplicity-free $G$ module.

Proof i) $\Leftrightarrow$ ii). The first implication is obvious, so we suppose that $\mathrm{k}(X)^{B}=\mathrm{k}$. By a theorem of Rosenlicht (see [13, Theorem 19.5]), $B$-orbits in general position can be separated by $B$-invariant functions: that is, there exists a $B$-stable affine open subset $U \subset X$ such that for all $x, y \in U$ with $B x \neq B y$ there exists $f \in \mathrm{k}(U)^{B}$ such that $f(x) \neq 0$ and $f(y)=0$. On the other hand $f$ must be constant, therefore $U$ is a single $B$-orbit.

ii) $\Rightarrow$ iii). Let $s_{1}, s_{2} \in \Gamma(X, \mathcal{L})^{(B)}$ be highest weight vectors of the same weight. Then $\frac{s_{1}}{s_{2}} \in \mathrm{k}(X)^{B}=\mathrm{k}$, hence $s_{1}$ and $s_{2}$ are proportional. It follows that the dimension of $\Gamma(X, \mathcal{L})_{\lambda}^{(B)}$ is 1 for all $\lambda \in \mathcal{X}(T)^{+}$, as claimed.

iii) $\Rightarrow$ ii). This is basically a consequence of the following fact: every normal quasi-projective $G$-variety can be embedded equivariantly in the projective space of a finite dimensional $G$-module (see [20, Corollary 2.6]). Let $\iota: X \hookrightarrow \mathbb{P}(V)$ be such an equivariant embedding and denote $\mathcal{L}=i^{*} \mathcal{O}_{\mathbb{P}(V)}(1)$. Then $\mathcal{L}$ is by construction a very ample and $G$-linearized line bundle on $X$, and for all $n \in \mathbb{N}$ the restriction to $X$ yields a $G$-equivariant homomorphism $\mathrm{S}^{n} V^{*} \rightarrow \Gamma\left(X, \mathcal{L}^{\otimes n}\right)$.

Let now $f \in \mathrm{k}(X)^{B}$, then there exist $n \in \mathbb{N}$ and $p, q \in \mathrm{S}^{n} V^{*}$ such that $f=\frac{p_{\mid X}}{q_{\mid X}}$. We may assume that $p, q$ are $B$-eigenvectors: indeed, the $B$-module $V^{\prime}=\left\{q^{\prime} \in\right.$ $\left.\mathrm{S}^{n} V^{*} \mid f q^{\prime} \in \mathrm{S}^{n} V^{*}\right\}$ is nonzero and finite dimensional, hence it contains a nonzero $B$-eigenvector by the Lie-Kolchin theorem (see e.g. [31, Theorem 6.3.1]). Since $f$ is $B$-invariant, if $p$ and $q$ are $B$-eigenvectors, then they have the same weight. On 
the other hand by assumption $\Gamma\left(X, \mathcal{L}^{\otimes n}\right)$ is a multiplicity-free $G$-module, thus $p_{\mid X}$ and $q_{\mid X}$ must be proportional, and $f$ must be constant.

iii') $\Rightarrow$ ii). If $X$ is quasi-affine, then $\mathrm{k}(X)$ is the quotient field of $\mathrm{k}[X]$. Let $f \in \mathrm{k}(X)^{B}$ and write $f$ as the ratio of two elements $p, q \in \mathrm{k}[X]$. Notice that we can assume $p, q \in \mathrm{k}[X]^{(B)}$. Let indeed $V$ be the subspace of $\mathrm{k}[X]$ generated by the $B$-orbit of $q$, then $V$ is finitely dimensional by Lemma 2.3 , and since it is $B$-stable by the Lie-Kolchin theorem it contains a $B$-eigenvector $q^{\prime}$. Write $q^{\prime}=\sum_{i} \xi_{i}\left(b_{i} . q\right)$ with $\xi_{i} \in \mathrm{k}$ and $b_{i} \in B$ and denote $p^{\prime}=\sum_{i} \xi_{i}\left(b_{i} \cdot p\right)$, then $f=b_{i} \cdot f=\frac{b_{i} \cdot p}{b_{i} \cdot q}$ for all $i$, hence $f=\frac{p^{\prime}}{q^{\prime}}$, and it follows that $p^{\prime}$ is $B$-semi-invariant as well. Clearly $p^{\prime}$ and $q^{\prime}$ must have the same weight, hence they are proportional because $\mathrm{k}[X]$ is multiplicity-free, and $f$ is constant.

By considering the normalization, one can restate the previous theorem for a larger class of $G$-varieties.

Definition 2.9. A $G$-variety is called multiplicity-free if it contains an open $B$ orbit.

Let $X$ be a $G$-variety and let $p: \widetilde{X} \rightarrow X$ be the normalization. Then the action $G \times X \rightarrow X$ induces a morphism $G \times \widetilde{X} \rightarrow X$. On the other hand $G \times \widetilde{X}$ is a normal variety, thus by the universal property we get a morphism $G \times \widetilde{X} \rightarrow \widetilde{X}$, which can be easily seen to be an action of $G$ on $\widetilde{X}$ making $p$ an equivariant morphism.

Notice that $X$ is multiplicity-free if and only if $\widetilde{X}$ is spherical. If moreover $X$ is quasi-projective (resp. quasi-affine), then $\widetilde{X}$ has the same property, and if $\mathcal{L}$ is a $G$-linearized line bundle on $X$, then the pullback $p^{*} \mathcal{L}$ is $G$-linearized as well and $\Gamma(X, \mathcal{L})$ is identified with a $G$-stable subspace of $\Gamma\left(\widetilde{X}, p^{*} \mathcal{L}\right)$. Therefore, we can restate the previous theorem as follows.

Theorem 2.10. Let $X$ be a $G$-variety and let $\tilde{X} \rightarrow X$ be the normalization, then the following are equivalent:

i) $X$ is multiplicity-free;

ii) Any B-invariant rational function on $X$ is constant;

iii) If $X$ is quasi-projective: for every $G$-linearized line bundle $\mathcal{L}$ on $\tilde{X}$, the space of global sections $\Gamma(\widetilde{X}, \mathcal{L})$ is a multiplicity-free $G$-module;

iii') If $X$ is quasi-affine: the coordinate ring $\mathrm{k}[X]$ is a multiplicity-free $G$ module.

Other characterizations of sphericality (or more generally of multiplicity-freeness) come from the study of the $G$-orbits and of the $B$-orbits. In particular we have the following.

Theorem 2.11. Let $X$ be a G-variety, then the following are equivalent:

i) $X$ is multiplicity-free;

ii) Every $G$-variety which is equivariantly birational to $X$ contains finitely many $G$-orbits;

iii) $X$ contains finitely many B-orbits.

The implication iii) $\Rightarrow$ i) is clear from the definition. For the implication i) $\Rightarrow$ ii), by taking the normalization we can assume that $X$ is normal, in which case the claim will be proved later (see Corollary 5.3). For the other implication, and for other characterizations as well, see [34, Section 25].

Every homogeneous $G$-variety $G / H$ is smooth, and it is quasi-projective. The latter claim follows by a theorem of Chevalley, stating that $G / H$ can be equivariantly embedded in the projective space of a finite dimensional rational $G$-module (see e.g. [31, Theorem 5.5.3]). Therefore Theorem 2.8 applies in particular to the 
case of homogeneous varieties. In that case, thanks to the description of the space of global sections of a linearized line bundle on a homogeneous space in Remark 1.1, we can also rephrase conditions iii) and iii') of Theorem 2.8 in more representation theoretical terms.

Theorem 2.12. Let $H \subset G$ be a closed subgroup, then the following are equivalent:

i) $G / H$ is spherical;

ii) For all $\chi \in \mathcal{X}(H)$, the $G$-module $\mathrm{k}[G]_{\chi}^{(H)}$ is multiplicity-free;

iii) For all $\lambda \in \mathcal{X}(T)^{+}$and for all $\chi \in \mathcal{X}(H)$, we have $\operatorname{dim} V^{*}(\lambda)_{\chi}^{(H)} \leqslant 1$;

iii') If $G / H$ is quasi-affine: for all $\lambda \in \mathcal{X}(T)^{+}$, we have $\operatorname{dim} V^{*}(\lambda)^{H} \leqslant 1$.

Proof i) $\Leftrightarrow$ ii). This is just a restatement of the equivalence i) $\Leftrightarrow$ iii) of Theorem 2.8 , by making use of Remark 1.1.

i) $\Leftrightarrow$ iii). If $k_{\chi}$ denotes the one-dimensional vector space with the action given by $\chi$, then by the Frobenius reciprocity together with Remark 1.1 we have

$$
\operatorname{Hom}_{G}\left(V(\lambda), \operatorname{Ind}_{H}^{G}(\chi)\right) \simeq \operatorname{Hom}_{H}\left(V(\lambda), \mathrm{k}_{\chi}\right)=V^{*}(\lambda)_{-\chi}^{(H)} .
$$

Therefore the claim follows again by the equivalence i) $\Leftrightarrow$ iii) of Theorem 2.8 , thanks to the isomorphism $\Gamma\left(G / H, \mathcal{L}_{\chi}\right) \simeq \operatorname{Ind}_{H}^{G}(\chi)$.

i) $\Leftrightarrow$ iii'). This follows as the previous equivalence by Theorem 2.8 , by making use of the equality $\mathrm{k}[G / H]=\mathrm{k}[G]^{H}$.

Definition 2.13. Given a $G$-variety $X$, define the weight lattice and the weight monoid of $X$ respectively as

$$
\begin{aligned}
\Lambda(X) & =\left\{\lambda \in \mathcal{X}(T) \mid \lambda \text { is the weight of a function in } \mathrm{k}(X)^{(B)}\right\}, \\
\Lambda^{+}(X) & =\left\{\lambda \in \mathcal{X}(T)^{+} \mid \lambda \text { is the weight of a function in } \mathrm{k}[X]^{(B)}\right\} .
\end{aligned}
$$

The rank of $\Lambda(X)$ is called the rank of $X$, denoted by rk $X$. For later use, we also denote $\mathcal{Q}(X)=\Lambda(X)_{\mathbb{Q}}^{\vee}$.

Remark 2.14. Suppose that $X$ is a multiplicity-free affine $G$-variety. Then $\Lambda^{+}(X)$ is a finitely generated monoid by Theorem 2.4 , and $\Lambda(X)=\mathbb{Z} \Lambda^{+}(X)$ : indeed the argument used to prove the implication iii') $\Rightarrow$ ii) in Theorem 2.8 shows that every $f \in \mathrm{k}(X)^{(B)}$ can be written as the ratio of two elements $p, q \in \mathrm{k}[X]^{(B)}$.

Notice that, if $X$ is spherical, then every function in $\mathrm{k}(X)^{(B)}$ is uniquely determined by its weight up to a constant: if indeed $f_{1}, f_{2} \in \mathrm{k}(X)^{(B)}$ have the same weight, then $f_{1} f_{2}^{-1} \in \mathrm{k}(X)^{B}=\mathrm{k}$. Given $f \in \mathrm{k}(G / H)^{(B)}$, we denote by $\lambda_{f} \in \Lambda(X)$ the corresponding weight. In particular we get an exact sequence

$$
1 \longrightarrow \mathrm{k}^{*} \longrightarrow \mathrm{k}(X)^{(B)} \longrightarrow \Lambda(X) \longrightarrow 0
$$

Given a submonoid $\Gamma \subset \Lambda_{\mathbb{Q}}$, define the saturation of $\Gamma$ as the monoid $\widetilde{\Gamma}=$ cone $(\Gamma) \cap \mathbb{Z} \Gamma$, and say that $\Gamma$ is saturated if $\widetilde{\Gamma}=\Gamma$.

Proposition 2.15. Let $X$ be a multiplicity-free affine $G$-variety and let $\widetilde{X}$ be its normalization, then $\widetilde{\Lambda^{+}}(X)=\Lambda^{+}(\widetilde{X})$.

Proof Let $\lambda \in \Lambda(X)$ be such that $n \lambda \in \Lambda^{+}(X)$ for some $n \in \mathbb{N}$, and let $f \in \mathrm{k}(X)^{(B)}$ of weight $\lambda$ : then $f^{n} \in \mathrm{k}(X)^{(B)}=\mathrm{k}(\widetilde{X})^{(B)}$ has weight $n \lambda$. Since $f^{n} \in \mathrm{k}[X]$, it follows that $f \in \mathrm{k}[\widetilde{X}]$, hence $\lambda \in \Lambda^{+}(\widetilde{X})$.

To show the other inclusion, we follow the argument given in [6, Proposition 2.8]. Let $I=\{f \in \mathrm{k}[X] \mid f \mathrm{k}[\tilde{X}] \subset \mathrm{k}[X]\}$ : since $\mathrm{k}[\widetilde{X}]$ is finitely generated over $\mathrm{k}[X]$, it follows that $I$ is a nonzero $G$-stable ideal, and $I^{U} \neq 0$ by the Lie-Kolchin theorem. Let $F \in I^{U} \backslash\{0\}$, then $F \mathrm{k}[\tilde{X}]^{U}$ is an ideal in $\mathrm{k}[X]^{U}$, which is a finitely generated k-algebra by Theorem 2.4. Hence $F \mathrm{k}[\widetilde{X}]^{U}$ is a finitely generated $\mathrm{k}[X]^{U}$-module. 
On the other hand as $\mathrm{k}[X]^{U}$-modules we have an isomorphism $\mathrm{k}[\tilde{X}]^{U} \simeq F \mathrm{k}[\tilde{X}]^{U}$, therefore $\mathrm{k}[X]^{U} \subset \mathrm{k}[\widetilde{X}]^{U}$ is an integral extension. that

Let now $\lambda \in \Lambda^{+}(\widetilde{X})$ and let $f \in \mathrm{k}[\widetilde{X}]_{\lambda}^{(B)}$. Then they exist $a_{1}, \ldots a_{n} \in \mathrm{k}[X]^{U}$ such

$$
f^{n}+a_{1} f^{n-1}+\ldots+a_{n-1} f+a_{n}=0 .
$$

Projecting on the weight space $\mathrm{k}[X]_{n \lambda}^{(B)}$, we may assume that $a_{m} \in \mathrm{k}[X]_{m \lambda}^{(B)}$ for all $m \leqslant n$. On the other hand some $a_{m}$ must be nonzero, therefore $m \lambda \in \Lambda^{+}(X)$.

Let $X$ be a multiplicity-free affine $G$-variety and let $\widetilde{X}$ be its normalization. If $\operatorname{char}(\mathrm{k})=0$, notice that the $G$-stable subalgebra $\mathrm{k}[X] \subset \mathrm{k}[\tilde{X}]$ is uniquely determined by the weight monoid of $X$ : indeed $\mathrm{k}[\tilde{X}]$ is a multiplicity-free $G$-module, and we can consider its decomposition into simple $G$-modules. Therefore we get the following corollary.

Corollary 2.16. Let $X$ be a multiplicity-free affine G-variety.

i) If $X$ is normal, then $\Lambda^{+}(X)$ is a saturated monoid.

ii) If $\operatorname{char}(\mathrm{k})=0$, then $X$ is normal if and only if $\Lambda^{+}(X)$ is saturated.

For later use, we also recall the following fact (see e.g. [34, Theorem 3.5]).

Theorem 2.17. Let $K$ be a solvable linear algebraic group and $K^{\prime} \subset K$ a closed subgroup, then $K / K^{\prime}$ is an affine variety.

Remark 2.18. In the notation of the previous theorem, notice that the following holds (see the proof of [34, Theorem 3.5] for more details): let $S \subset K$ and $S^{\prime} \subset K^{\prime}$ be maximal diagonalizable subgroups and suppose that $S^{\prime} \subset S$, then the multiplication induces a $S$-equivariant isomorphism

$$
S \times{ }^{S^{\prime}} K_{\mathrm{u}} / K_{\mathrm{u}}^{\prime} \stackrel{\sim}{\longrightarrow} K / K^{\prime} .
$$

Therefore $K / K^{\prime}$ is affine because $S \times K_{\mathrm{u}} / K_{\mathrm{u}}^{\prime}$ is affine, and

$$
S \times S^{\prime} K_{\mathrm{u}} / K_{\mathrm{u}}^{\prime}=\left(S \times K_{\mathrm{u}} / K_{\mathrm{u}}^{\prime}\right) / / S^{\prime}
$$

is the categorical quotient of an affine variety by a reductive group. Moreover, notice that $\mathrm{k}\left(K / K^{\prime}\right)^{(K)}=\mathrm{k}\left(S / S^{\prime}\right)^{(S)}$ : indeed every $f \in \mathrm{k}\left(K / K^{\prime}\right)^{(K)}$ is constant on the fiber $K_{\mathrm{u}} / K_{\mathrm{u}}^{\prime}$, and the projection $S \times{ }^{S^{\prime}} K_{\mathrm{u}} / K_{\mathrm{u}}^{\prime} \rightarrow S / S^{\prime}$ induces an inclusion $\mathrm{k}\left(S / S^{\prime}\right)^{(S)} \subset \mathrm{k}\left(K / K^{\prime}\right)^{(K)}$.

We can apply previous discussion in the case of a spherical variety as follows. Suppose that $X$ is a spherical $G$-variety with open $B$-orbit $B x_{0}$ and let $K$ be the stabilizer of $x_{0}$ in $B$. Then $\mathrm{k}(X)^{(B)}=\mathrm{k}\left(B x_{0}\right)^{(B)}=\mathrm{k}(B / K)^{(B)}$, therefore $\Lambda(X)$ is naturally identified with $\mathcal{X}(T / S)$, where $S \subset K$ is any maximal diagonalizable subgroup and $T \subset B$ is any maximal torus containing $S$.

Let $X$ be a spherical $G$-variety and denote

$$
\begin{gathered}
\mathcal{D}(X)=\{B \text {-stable prime divisors of } X\}, \\
\Delta(X)=\{D \in \mathcal{D}(X) \mid D \text { is not } G \text {-stable }\} .
\end{gathered}
$$

If moreover $Y \subset X$ is a $G$-orbit, then we denote

$$
\begin{gathered}
\mathcal{D}_{Y}(X)=\{D \in \mathcal{D}(X) \mid Y \subset D\}, \\
\Delta_{Y}(X)=\Delta(X) \cap \mathcal{D}_{Y}(X) .
\end{gathered}
$$

Definition 2.19. The elements of $\Delta(X)$ are called the colors of $X$.

Proposition 2.20. $\mathcal{D}(X)$ is the set of the irreducible components of $X \backslash B x_{0}$. In particular $\mathcal{D}(X)$ is a finite set, and

$$
\Delta(X)=\left\{D \in \mathcal{D}(X) \mid D \cap G x_{0} \neq \varnothing\right\} .
$$


Proof By Theorem 2.17, the open $B$-orbit of $X$ is an affine variety, thus its complement has pure codimension one (see [12, Exercise 12.18] or [14, Proposition II.3.1]).

Thanks to the sequence $(2.1)$, every $D \in \mathcal{D}(X)$ defines a homomorphism $\rho_{X}(D)$ : $\Lambda(X) \rightarrow \mathbb{Z}$ by setting

$$
\left\langle\rho_{X}(D), \lambda\right\rangle=\nu_{D}\left(f_{\lambda}\right)
$$

where $f_{\lambda} \in \mathrm{k}(X)^{(B)}$ is any eigenfunction of weight $\lambda$ and where $\nu_{D}\left(f_{\lambda}\right)$ is the order of vanishing of $f$ along $D$. Therefore we get a map

$$
\rho_{X}: \Delta(X) \longrightarrow \mathcal{Q}(X) \text {. }
$$

(when the variety $X$ is clear from the context, we will often drop the index).

\section{Examples}

We present in this section some examples of spherical varieties, and compute the corresponding weight lattices, weight monoids and sets of colors. Even if not always needed, throughout this section we will assume for convenience that $\operatorname{char}(\mathrm{k})=0$ : in particular, this will allow us to use the Peter-Weyl theorem (see e.g. [11, Theorem 4.2.3]), namely the decomposition into $G \times G$-modules

$$
\mathrm{k}[G] \simeq \bigoplus_{\lambda \in \mathcal{X}(T)^{+}} V(\lambda) \otimes V^{*}(\lambda),
$$

which fails in positive characteristic.

Example 3.1. Suppose that $X$ is a toric $T$-variety, where $T$ is an algebraic torus of dimension $n$. Then $X$ contains an open $T$-orbit isomorphic to $T$, and if $n=\operatorname{dim}(X)$ then $\Lambda(X) \simeq \mathbb{Z}^{n}$. Notice that in this case there are no colors: indeed $T=B=G$, therefore every $B$-stable divisor is $G$-stable.

Example 3.2. Suppose that $G / P$ is a flag variety, then $\Lambda(G / P)=0$. Indeed, $U$ has an open orbit in $G / P$. Moreover, every rational $B$-eigenfunction on $G / P$ is $U$-invariant, hence it must be constant. Suppose for simplicity that $P=B$ : then the $B$-orbits in $G / B$ are the Schubert cells, and by the Bruhat decomposition they are in correspondence with the elements of the Weyl group of $G$. The colors are the Schubert divisors, and they are in correspondence with the simple roots of $G$.

Example 3.3. Let $T \subset \mathrm{SL}_{2}$ be the subgroup of diagonal matrices, let $B \subset \mathrm{SL}_{2}$ be the subgroup of upper triangular matrices and let $\alpha=2 \omega_{\alpha}$ be the corresponding positive root. Set $\dot{s}_{\alpha}=\left(\begin{array}{cc}0 & 1 \\ -1 & 0\end{array}\right)$ and $u_{\alpha}=\left(\begin{array}{ll}1 & 1 \\ 0 & 1\end{array}\right)$, then $\mathrm{SL}_{2}$ decomposes into double cosets with respect to the action of $B \times T$ as follows:

$$
\mathrm{SL}_{2}=B \cup B \dot{s}_{\alpha} \cup B \dot{s}_{\alpha} u_{\alpha} T \text {. }
$$

Therefore $\mathrm{SL}_{2} / T$ is spherical, since it decomposes into three $B$-orbits: the open orbit $B \dot{s}_{\alpha} u_{\alpha} T / T$, and the two $B$-stable divisors $D^{+}=B / T$ and $D^{-}=B \dot{s}_{\alpha} / T$.

To compute the weight lattice, by the Peter-Weyl theorem it follows

$$
\mathrm{k}\left[\mathrm{SL}_{2} / T\right]=\mathrm{k}\left[\mathrm{SL}_{2}\right]^{T}=\bigoplus_{m \in \mathbb{N}} V\left(m \omega_{\alpha}\right) \otimes V^{*}\left(m \omega_{\alpha}\right)^{T}=\bigoplus_{m \in \mathbb{N}} V(m \alpha)
$$

Therefore $\Lambda^{+}\left(\mathrm{SL}_{2} / T\right)=\mathbb{N} \alpha$, and since $\mathrm{SL}_{2} / T$ is affine we get by Remark 2.14 that $\Lambda\left(\mathrm{SL}_{2} / T\right)=\mathbb{Z} \alpha$.

We now compute the map $\rho: \Delta\left(\mathrm{SL}_{2} / T\right) \rightarrow \mathcal{Q}\left(\mathrm{SL}_{2} / T\right)$. For $1 \leqslant i, j \leqslant 2$, let $a_{i j} \in \mathrm{k}\left[\mathrm{SL}_{2}\right]$ be the corresponding matrix coefficient, then $B=\operatorname{div}\left(a_{21}\right)$ and $B \dot{s}_{\alpha}=\operatorname{div}\left(a_{22}\right)$. Let $V=\mathrm{k}[x, y]_{2}$ be the space of homogeneous polynomials in $x, y$ of degree 2, regarded as a $\mathrm{SL}_{2}$-module with the linear action on the coordinates given by $g .\left(\begin{array}{l}x \\ y\end{array}\right) \mapsto g^{-1}\left(\begin{array}{l}x \\ y\end{array}\right)$. Fix the basis $x^{2}, x y, y^{2}$ and let $e_{20}, e_{11}, e_{02}$ be the dual basis in $V^{*}$. Since $x y \in V^{T}$ we obtain a function $f \in \mathrm{k}\left[\mathrm{SL}_{2} / T\right]$ by setting 
$f(g)=\left\langle e_{20}, g \cdot x y\right\rangle$. Notice that $\operatorname{div}(f)=D^{+}+D^{-}$: indeed, regarding $f$ as a function on $\mathrm{SL}_{2}$, we have $f=-a_{21} a_{22}$. On the other hand $e_{20} \in\left(V^{*}\right)^{(B)}$ is a highest weight function of weight $\alpha$, therefore $f \in \mathrm{k}\left[\mathrm{SL}_{2} / T\right]^{(B)}$ also has weight $\alpha$ and we get $\left\langle\rho\left(D^{+}\right), \alpha\right\rangle=\nu_{D^{+}}(f)=1$ and $\left\langle\rho\left(D^{-}\right), \alpha\right\rangle=\nu_{D^{+}}(f)=1$. Since $\Lambda\left(\mathrm{SL}_{2} / T\right)=\mathbb{Z} \alpha$, in particular we get the equalities

$$
\rho\left(D^{+}\right)=\rho\left(D^{-}\right)=\frac{1}{2} \alpha^{\vee} .
$$

Example 3.4 (Symmetric matrices, part II). Let $X=\mathrm{Sym}_{n}$ be the space of symmetric matrices of order $n$ with the action of $G=\mathrm{GL}_{n}$, and keep the notation of Example $2.2 \mathrm{v}$ ).

By the classification of the quadratic forms up to congruence, the $G$-orbits in $X$ are parametrized by the rank. More precisely, for $i \leqslant n$, let $X_{i}=\{x \in X \mid \operatorname{rk}(x) \leqslant$ $i$ \} and let $x_{i} \in X_{i}$ be the rank $i$ diagonal matrix which is the identity in the upper left block and zero everywhere else. Then the $G$-orbits are precisely $G x_{0}, \ldots, G x_{n}$, and $X_{i}=\overline{G x_{i}}$ for all $i \leqslant n$. Therefore we have the following inclusions of $G$-orbit closures:

$$
0=G x_{0} \subset \overline{G x_{1}} \subset \ldots \subset \overline{G x_{n}} .
$$

Let $d_{k}$ be the determinant of the upper left square block of order $k$, and notice that $d_{k} \in \mathrm{k}[X]^{U}$. Moreover $d_{k} \in \mathrm{k}[X]^{(T)}$, where $T \subset \mathrm{GL}_{n}$ is the torus of diagonal matrices, and for $t=\left(t_{1}, \ldots, t_{n}\right) \in T$ it holds

$$
\left(t . d_{k}\right)\left(\left[a_{i j}\right]\right)=d_{k}\left(t^{-1} \cdot\left[a_{i j}\right]\right)=d_{k}\left(\left[t_{i} t_{j} a_{i j}\right]\right)=t_{1}^{2} \cdots t_{k}^{2} d_{k}\left(\left[a_{i j}\right]\right) .
$$

If $\varepsilon_{i} \in \mathcal{X}(T)$ denotes the projection on the $i$-th coordinate of $T$ and $\omega_{i}$ denotes the highest weight of $\mathrm{GL}_{n}$ in $\wedge^{i} \mathrm{k}^{n}$, we get then

$$
\lambda_{d_{k}}=2 \varepsilon_{1}+\ldots+2 \varepsilon_{k}=2 \omega_{k} .
$$

On the other hand, notice that $\Lambda^{+}(X) \subset 2 \mathcal{X}(T)^{+}$. Let indeed $\chi \in \Lambda^{+}(X)$, write $\chi=\sum a_{i} \varepsilon_{i}$ and let $t(i)=\operatorname{diag}(1, \ldots, 1,-1,1, \ldots, 1)$ be the diagonal matrix whose unique negative entry is in position $i$ : then $t(i)^{-1} \cdot x_{n}=x_{n}$. Since $B x_{n}$ is open in $X$, it follows $-1^{a_{i}}=\chi(t(i))=1$, hence $a_{i}$ is even. It follows that $\Lambda^{+}(X)=2 \mathcal{X}(T)^{+}$, and by Remark 2.14 we get the equality $\Lambda(X)=2 \mathcal{X}(T)$. Since $\mathrm{k}[X]$ is a multiplicity-free $G$-module and $\Lambda^{+}(X)$ is a free monoid, it follows moreover that $\mathrm{k}[X]^{U}=\mathrm{k}\left[d_{1}, \ldots, d_{n}\right]$ is a polynomial ring, and $\mathrm{k}[X]^{(B)}$ is the set of monomials therein.

Since $d_{k} \in \mathrm{k}[X]^{(B)}$ and $d_{k}\left(x_{n}\right)=1$, we have $B x_{n} \subset\left\{x \in X \mid d_{k}(x) \neq 0\right\}$ for all $i \leqslant n$. On the other hand one can actually check that

$$
B x_{n}=\bigcap_{i=1}^{n}\left\{x \in X \mid d_{k}(x) \neq 0\right\},
$$

and being determinants the polynomials $d_{k}$ are irreducible. Therefore $X \backslash B x_{n}$ is the union of the irreducible hypersurfaces $D_{1}, \ldots, D_{n}$ defined by $d_{1}, \ldots, d_{n}$, namely $\mathcal{D}(X)=\left\{D_{1}, \ldots, D_{n}\right\}$. On the other hand $d_{n}$ is the determinant, therefore $D_{n}=$ $X_{n-1}$ is the unique $G$-stable divisor and we get $\Delta(X)=\left\{D_{1}, \ldots, D_{n-1}\right\}$.

By the irreducibility of the $d_{k}$, it follows that $\nu_{D_{k}}\left(d_{h}\right)=\delta_{h k}$ is the Kronecker delta whenever $1 \leqslant h, k \leqslant n$. Therefore we get $\rho\left(D_{k}\right)=\frac{1}{2} \alpha_{k}^{\vee}$ for all $k<n$ and $\rho\left(D_{n}\right)=\frac{1}{2} \omega_{n}^{*}$, where $\left\{\alpha_{1}^{\vee}, \ldots, \alpha_{n-1}^{\vee}, \omega_{n}^{*}\right\} \subset \mathcal{X}(T)^{\vee}$ is the dual basis of $\left\{\omega_{1}, \ldots, \omega_{n}\right\}$.

Example 3.5 (Determinantal varieties, part II). Keep the notation of Example $2.2 \mathrm{vi}$ ), and denote by $U_{m}^{-}$(resp. $U_{n}$ ) the unipotent subgroup of lower triangular (resp. upper triangular) matrices in $\mathrm{GL}_{m}$ (resp. in $\mathrm{GL}_{n}$ ) and denote $U=U_{m}^{-} \times U_{n}$. As in the previous example, the $G$-orbits in $M_{m, n, r}$ are parametrized by the rank. 
By the dimension formula of a determinantal variety, notice that $M_{m, n, r}$ contains a $G$-stable divisor if and only if $m=n=r$.

Given $x \in M_{m, n, r}$ and $k \leqslant r$, we denote by $d_{k}$ the determinant of the upper left square block of order $k$. It is easily seen that $d_{1}, \ldots, d_{r} \in \mathrm{k}\left[M_{m, n, r}\right]^{U}$. We now show the equality

$$
\mathrm{k}\left[M_{m, n, r}\right]^{U}=\mathrm{k}\left[d_{1}, \ldots, d_{r}\right]
$$

by showing that $\Lambda^{+}\left(M_{m, n, r}\right)$ is freely generated by the weights $\omega_{1}^{\prime}-\omega_{1}, \ldots, \omega_{r}^{\prime}-\omega_{r}$.

Let $T_{m}$ (resp. $\left.T_{n}\right)$ be the maximal torus of $B_{m}^{-}\left(\right.$resp. of $\left.B_{n}\right)$ and set $T=T_{m} \times T_{n}$. Let $t=\left(t_{1}, \ldots, t_{m}, s_{1}, \ldots, s_{n}\right) \in T$, then $d_{k} \in \mathrm{k}\left[M_{m, n, r}\right]^{(T)}$ and we have

$$
\left((t, s) \cdot d_{k}\right)\left(\left[a_{i j}\right]\right)=d_{k}\left(\left(t^{-1}, s^{-1}\right) \cdot\left[a_{i j}\right]\right)=d_{k}\left(\left[t_{i}^{-1} s_{j} a_{i j}\right]\right)=t_{1}^{-1} s_{1} \cdots t_{k}^{-1} s_{k} d_{k}\left(\left[a_{i j}\right]\right) .
$$

If $\varepsilon_{i} \in \mathcal{X}\left(T_{m}\right)$ (resp. $\varepsilon_{i}^{\prime} \in \mathcal{X}\left(T_{n}\right)$ ) denotes the projection on the $i$-th coordinate of $T_{m}$ (resp. of $T_{n}$ ) and if $\omega_{i}$ (resp. $\omega_{i}^{\prime}$ ) denotes the highest weight of $\mathrm{GL}_{m}$ in $\wedge^{i} \mathrm{k}^{m}$ (resp. of $\mathrm{GL}_{n}$ in $\Lambda^{i} \mathrm{k}^{n}$ ), we get then

$$
\lambda_{d_{k}}=\varepsilon_{1}^{\prime}-\varepsilon_{1}+\ldots+\varepsilon_{k}^{\prime}-\varepsilon_{k}=\omega_{k}^{\prime}-\omega_{k} .
$$

Decompose $T_{m}=T_{r} \times T_{m-r}$ and $T_{n}=T_{r} \times T_{n-r}$, and notice that $\operatorname{diag}\left(T_{r}\right) \times$ $T_{m-r} \times T_{n-r}$ fixes $x_{0}$. If $f \in \mathrm{k}\left[M_{m, n, r}\right]^{(B)}$ has weight $\lambda_{f}=\left(\lambda_{1}, \lambda_{2}, \lambda_{3}, \lambda_{4}\right) \in$ $\mathcal{X}\left(T_{r}\right) \times \mathcal{X}\left(T_{m-r}\right) \times \mathcal{X}\left(T_{r}\right) \times \mathcal{X}\left(T_{n-r}\right)$, it follows that $\left(\lambda_{1}+\lambda_{3}\right)_{\mid \operatorname{diag}\left(T_{r}\right)}=\lambda_{2}=\lambda_{4}=0$, consequently

$$
\Lambda^{+}\left(M_{m, n, r}\right) \subset\left\langle\varepsilon_{1}^{\prime}-\varepsilon_{1}, \ldots, \varepsilon_{r}^{\prime}-\varepsilon_{r}\right\rangle_{\mathbb{Z}}
$$

On the other hand every weight in $\Lambda^{+}\left(M_{m, n, r}\right)$ is dominant with respect to $B_{m}^{-} \times B_{n}$, and since $d_{k}$ is not invertible of weight $\omega_{k}^{\prime}-\omega_{k}$ it follows that

$$
\Lambda^{+}\left(M_{m, n, r}\right)=\left\langle\omega_{1}^{\prime}-\omega_{1}, \ldots, \omega_{r}^{\prime}-\omega_{r}\right\rangle_{\mathbb{N}} .
$$

Finally, by Remark 2.14 we get the equality

$$
\Lambda\left(M_{m, n, r}\right)=\left\langle\omega_{1}^{\prime}-\omega_{1}, \ldots, \omega_{r}^{\prime}-\omega_{r}\right\rangle_{\mathbb{Z}}
$$

Notice that the weight monoid $\Lambda^{+}\left(M_{m, n, r}\right)$ is saturated, thus $M_{m, n, r}$ is a normal variety by Corollary 2.16 (this holds true in all characteristics, see [8, Proposition $1.4 \mathrm{c}])$. Therefore $M_{m, n, r}$ is an affine spherical variety.

Example 3.6 (Reductive groups, part I). Recall from Example 2.2 iv) that $G$ is a spherical $(G \times G)$-variety, and fix $B^{-} \times B$ as a Borel subgroup of $G \times G$. Let $f \in \mathrm{k}[G]^{\left(B^{-} \times B\right)}$ and write $\lambda_{f}=\left(\lambda_{1}, \lambda_{2}\right)$ with $\lambda_{1}, \lambda_{2} \in \mathcal{X}(T)$ : since $\operatorname{diag}(G)$ fixes the identity of $G$ and since $B^{-} B \subset G$ is open, it follows that $\lambda_{1}+\lambda_{2}=0$. Conversely, recall that the multiplication induces an isomorphism $B^{-} B \simeq U^{-} \times T \times U$ (see [31, Theorem 6.3.5 and Lemma 8.3.6]. Therefore, for all $\lambda \in \mathcal{X}(T)$, we get a function $f_{\lambda} \in \mathrm{k}(G)^{\left(B^{-} \times B\right)}=\mathrm{k}\left(B^{-} B\right)^{\left(B^{-} \times B\right)}$ of weight $(-\lambda, \lambda)$ by setting $f_{\lambda}\left(u t u^{\prime}\right)=\lambda(t)$ for all $t \in T, u \in U^{-}$and $u^{\prime} \in U$. Hence we have the following description:

$$
\Lambda(G)=\{(-\lambda, \lambda) \mid \lambda \in \mathcal{X}(T)\} .
$$

When $\lambda \in \mathcal{X}(T)^{+}$, we can also give a more representation theoretical description of the function $f_{\lambda}$. Let $v_{\lambda} \in V(\lambda)^{(B)}$ be a highest weight vector and $\varphi_{\lambda} \in V^{*}(\lambda)^{\left(B^{-}\right)}$ be a lowest weight vector, and define $f_{\lambda}(g)=\left\langle\varphi_{\lambda}, g v_{\lambda}\right\rangle$ : then $f_{\lambda} \in \mathrm{k}[G]^{\left(B^{-} \times B\right)}$ has weight $(-\lambda, \lambda)$, and up to a normalization $f_{\lambda}\left(u t u^{\prime}\right)=\lambda(t)$ for all $t \in T, u \in U^{-}$ and $u^{\prime} \in U$.

We now describe the colors of $G$, and the map $\rho: \Delta(G) \rightarrow \Lambda(G)$. By the Bruhat decomposition, the colors coincide with the Schubert divisors $D_{\alpha}=\overline{B^{-} \dot{s}_{\alpha} B}$, where $\alpha$ is a simple root and where $\dot{s}_{\alpha} \in \mathrm{N}_{G}(T)$ is a representative for the corresponding simple reflection $s_{\alpha} \in W$. Therefore $\Delta(G)$ is identified with the set of simple roots of $G$. Up to replacing $G$ with a finite covering, we can assume that $\operatorname{Pic}(G)=0$ (see 
[20, Proposition 4.6]). In particular, every divisor on $G$ is principal, or equivalently $\mathrm{k}[G]$ is a factorial ring (see [12, Proposition B.72]).

Notice that $D_{\alpha}$ has an equation in $\mathrm{k}[G]$ which is $\left(B^{-} \times B\right)$-semi-invariant of weight $\left(-\omega_{\alpha}, \omega_{\alpha}\right)$, where $\omega_{\alpha}$ denotes the fundamental weight associated to $\alpha$. Indeed, let $P_{\alpha} \subset G$ be the maximal parabolic subgroup containing $B$ associated to $\alpha$ and let $X_{\alpha}=\overline{B^{-} s_{\alpha} P_{\alpha}} / P_{\alpha}$ be the unique Schubert divisor of $G / P_{\alpha}$. Then the line bundle $\mathcal{O}\left(X_{\alpha}\right) \in \operatorname{Pic}\left(G / P_{\alpha}\right)$ is very ample and induces a closed equivariant embedding $G / P_{\alpha} \subset \mathbb{P}\left(V\left(\omega_{\alpha}\right)\right)$, and the lowest weight vector $\varphi_{\omega_{\alpha}}$ is the canonical section of $X_{\alpha}$ in $\Gamma\left(G / P_{\alpha}, \mathcal{O}\left(X_{\alpha}\right)\right)=V^{*}\left(\omega_{\alpha}\right)$. In particular, composing with the projection $G \rightarrow G / P_{\alpha}$, it follows that the function $f_{\omega_{\alpha}}$ that we defined above is an equation for $D_{\alpha}$. Finally, notice that $\rho\left(D_{\alpha}\right)=\left(-\alpha^{\vee}, \alpha^{\vee}\right)$, where $\alpha^{\vee}$ is the coroot associated to $\alpha$. Indeed, $\mathrm{k}[G]$ is a factorial ring and a multiplicity-free $(G \times G)$ module, therefore, up to a scalar factor, for all $\lambda \in \mathcal{X}(T)$ we have an equality of the shape $f_{\lambda}=\lambda_{0} \prod f_{\omega_{\alpha}}^{\left\langle\lambda, \alpha^{\vee}\right\rangle}$ for some $\lambda_{0} \in \mathcal{X}(G)$.

Example 3.7. Recall from Example 2.2 ii) that $G / U$ is a spherical variety. Then $\mathrm{k}[G / U]^{U}=\mathrm{k}[G]^{U \times U}$, thus by the Peter-Weyl theorem

$$
\mathrm{k}[G / U]^{U} \simeq \bigoplus_{\lambda \in \mathcal{X}(T)^{+}} V(\lambda)^{U} \otimes V^{*}(\lambda)^{U}
$$

and we get $\Lambda^{+}(G / U)=\mathcal{X}(T)^{+}$. Since $\Lambda(G / U)$ is a lattice containing $\Lambda^{+}(G / U)$, it follows that $\Lambda(G / U)=\mathcal{X}(T)$.

As in Examples 3.2 and 3.6, the $B$-orbits and the colors in $G / U$ are described in terms of Weyl group elements by making use of the Bruhat decomposition. In particular, the colors are parametrized by the simple roots of $G$ associated to $B$ : if $w_{0} \in W$ is the longest element, then the colors are the orbit closures of the shape $D_{\alpha}=\overline{B \dot{s}_{\alpha} w_{0} B / U}$, where $\alpha$ is a simple root and where $\dot{s}_{\alpha} \in \mathrm{N}_{G}(T)$ is a representative for the corresponding simple reflection $s_{\alpha} \in W$. Reasoning as in Example 3.6, one checks that $\rho\left(D_{\alpha}\right)=\alpha^{\vee}$.

\section{InVARIANT VALUATIONS ON A SPHERICAL HOMOGENEOUS SPACE}

Definition 4.1. Let $X$ be a normal variety. A valuation on $X$ is a map $\nu: \mathrm{k}(X) \rightarrow$ $\mathbb{Q} \cup\{+\infty\}$ with the following properties, whenever $f_{1}, f_{2} \in \mathrm{k}(X)$ :

i) $\nu\left(f_{1}+f_{2}\right) \geqslant \min \left\{\nu\left(f_{1}\right), \nu\left(f_{2}\right)\right\}$;

ii) $\nu\left(f_{1} f_{2}\right)=\nu\left(f_{1}\right)+\nu\left(f_{2}\right)$;

iii) $\left.\nu\right|_{\mathrm{k}^{*}}=0, \nu(0)=+\infty$ and $\nu\left(\mathrm{k}(X)^{*}\right) \subset \mathbb{Q}$.

If moreover $X$ is a $G$-variety, then a valuation $\nu$ is called invariant if $\nu(g . f)=\nu(f)$ for all $g \in G$.

We denote by $\mathcal{V}(X)$ the set of invariant valuations on a normal $G$-variety $X$.

Example 4.2. Let $D$ be a prime divisor on a normal variety $X$, then $D$ defines a valuation $\nu_{D}$, which associates to any function $f \in \mathrm{k}(X)$ its order along $D$ (for a detailed treatment, we refer to [12, Section 11.3]). Indeed, since it is normal, $X$ is smooth in codimension one, and the local ring $\mathcal{O}_{X, D}$ of the rational functions defined on $D$ is a discrete valuation ring with quotient field $\mathrm{k}(X)$. In particular, the maximal ideal $\mathfrak{m}_{X, D} \subset \mathcal{O}_{X, D}$ of the rational functions vanishing identically on $D$ is principal, generated by any irreducible element $z \in \mathfrak{m}_{X, D}$. Therefore every function $f \in \mathrm{k}(X)$ can be written in the form $z^{n} \frac{f_{1}}{f_{2}}$ with $n \in \mathbb{Z}$ and $f_{1}, f_{2} \in \mathcal{O}_{X, D} \backslash \mathfrak{m}_{X, D}$. By definition $\nu_{D}(f)=n$.

Given a valuation $\nu$ of a normal variety $X$, we define

$$
\mathcal{O}_{\nu}=\{f \in \mathrm{k}(X) \mid \nu(f) \geqslant 0\}, \quad \mathfrak{m}_{\nu}=\{f \in \mathrm{k}(X) \mid \nu(f)>0\} .
$$


Notice that $\mathcal{O}_{\nu}$ is a local ring with maximal ideal $\mathfrak{m}_{\nu}$. We say that a closed subvariety $Y \subset X$ is a center of $\nu$ if $\mathcal{O}_{X, Y} \subset \mathcal{O}_{\nu}$ and $\mathfrak{m}_{X, Y} \subset \mathfrak{m}_{\nu}$.

Proposition 4.3. Let $\nu$ be a valuation on a normal variety $X$.

i) If $X$ is affine, then $\nu$ has a center on $X$ if and only if $\nu_{\mid \mathrm{k}[X]} \geqslant 0$. In this case the center is unique, defined by the ideal $\mathfrak{m}_{\nu} \cap \mathrm{k}[X]$.

ii) The center of $\nu$ is unique, if it exists.

iii) If $X$ is a $G$-variety and $\nu \in \mathcal{V}(X)$, then the center of $\nu$ is $G$-stable.

Proof i) Suppose that $Y$ is a center for $\nu$, then $\mathrm{k}[X] \subset \mathcal{O}_{X, Y} \subset \mathcal{O}_{\nu}$, therefore $\nu_{\mid \mathrm{k}[X]} \geqslant 0$. Suppose conversely that $\nu_{\mid \mathrm{k}[X]} \geqslant 0$, and let $Y$ be the subvariety of $X$ defined by the prime ideal $\mathfrak{m}_{\nu} \cap \mathrm{k}[X]$. This is clearly a center for $\nu$, suppose that $Y^{\prime}$ is another center. Then the ideal of $Y^{\prime}$ in $\mathrm{k}[X]$ is contained $\mathfrak{m}_{\nu}$, hence in the ideal of $Y$ and it follows that $Y^{\prime} \subset Y$. Suppose that $f \in \mathrm{k}[X]$ vanishes on $Y^{\prime}$, but not on $Y$ : then $f$ is invertible in $\mathcal{O}_{X, Y} \subset \mathcal{O}_{\nu}$, but $f \in \mathfrak{m}_{X, Y^{\prime}} \subset \mathfrak{m}_{\nu}$, absurd. Therefore $Y=Y^{\prime}$ must hold.

ii) Suppose that $Y, Y^{\prime}$ are both centers for $\nu$. Then for every affine open subset $U \subset X$ the intersections $Y \cap U$ and $Y^{\prime} \cap U$ are both centers for $\nu$ on $U$, hence they coincide by i). Therefore $Y=Y^{\prime}$ since we can cover $X$ with affine open subsets.

iii) Let $Y$ be the center of $\nu$. Since $\nu$ is invariant it follows that $g . Y$ is also a center of $\nu$, therefore $g . Y=Y$ because the center is unique by ii).

Remark 4.4. Let $X$ be a normal variety, then every subvariety $Y \subset X$ is the center of a valuation on $X$. This is clear if $Y$ is a divisor, so we can $\operatorname{assume} \operatorname{codim}(Y)>1$. Let $p: \widetilde{X}_{Y} \rightarrow X$ be the normalization of the blow up of $X$ along $Y$, then we can consider any irreducible component $Z \subset p^{-1}(Y)$ of the exceptional divisor, and since $\mathrm{k}\left(\widetilde{X}_{Y}\right)=\mathrm{k}(X)$ the corresponding valuation $\nu_{Z}$ is a valuation on $X$ with center $Y$. Notice that $\nu_{Z}$ is invariant if $Y$ is $G$-stable: therefore every $G$-invariant subvariety of $X$ is the center of an invariant valuation.

The following proposition is due to Sumihiro (see [32, Lemma 10 and 11])

Proposition 4.5 ([24, Lemma 3.2], [17, Lemma 2.4]). Let $\nu$ be a valuation on $G$, then there exists a unique $\bar{\nu} \in \mathcal{V}(G)$ such that $\bar{\nu}(f)=\nu(g . f)$ for all $f \in \mathrm{k}(G)$ and for all $g$ in a nonempty open subset $U_{f} \subset G$.

Proof We show that, for all $f \in \mathrm{k}(G)$, the value $\nu(g . f)$ is constant for $g$ in a nonempty open subset $U_{f} \subset G$. Once this will be proved, the claim will follow because we can define $\bar{\nu}(f)=\nu(g . f)$, where $g \in U_{f}$. This actually defines a valuation on $G$ : if indeed $g \in U_{f_{1}} \cap U_{f_{2}} \cap U_{f_{1}+f_{2}}$ (which is not empty) then we get

$$
\begin{gathered}
\bar{\nu}\left(f_{1}+f_{2}\right)=\nu\left(g \cdot f_{1}+g \cdot f_{2}\right) \geqslant \min \left(\nu\left(g \cdot f_{1}\right), \nu\left(g \cdot f_{2}\right)\right)=\min \left(\bar{\nu}\left(f_{1}\right), \bar{\nu}\left(f_{2}\right)\right), \\
\bar{\nu}\left(f_{1} f_{2}\right)=\nu\left(\left(g \cdot f_{1}\right)\left(g \cdot f_{2}\right)\right)=\nu\left(g \cdot f_{1}\right)+\nu\left(g \cdot f_{2}\right)=\bar{\nu}\left(f_{1}\right)+\bar{\nu}\left(f_{2}\right) .
\end{gathered}
$$

Moreover $\bar{\nu} \in \mathcal{V}(G)$, because for $g \in G$ we can take $U_{g . f}=U_{f} g^{-1}$.

To show that $\nu(g . f)$ is constant on a nonempty open subset of $G$, we may assume that $f \in \mathrm{k}[G]$. For $n \in \mathbb{Q}$ define $V(n)=\left\{f^{\prime} \in \mathrm{k}(G) \mid \nu\left(f^{\prime}\right) \geqslant n\right\}$. Вy Lemma 2.3, the orbit $G f$ generates a finite dimensional submodule $M \subset \mathrm{k}[G]$. Let $f_{1}, \ldots, f_{m}$ be a basis of $M$, and denote $n_{0}=\min _{i} \nu\left(f_{i}\right)$. Then $M \subset V\left(n_{0}\right)$, and $M^{\prime}=\left\{f^{\prime} \in M \mid \nu\left(f^{\prime}\right)>n_{0}\right\}$ is a proper subspace of $M$. Denote

$$
U_{f}=\left\{g \in G \mid g . f \notin M^{\prime}\right\}:
$$

then $U_{f}$ is a nonempty open subset of $G$, and $\nu(g . f)=n_{0}$ for all $g \in U_{f}$.

Corollary 4.6 ([17, Corollary 2.5]). Let $H \subset G$ be a closed subgroup. If $\nu \in$ $\mathcal{V}(G / H)$, then there exists $\bar{\nu} \in \mathcal{V}(G)$ whose restriction to $\mathrm{k}(G / H)$ is $\nu$. 
Proof By a general result in valuation theory (see e.g. [12, Proposition B.69]), we can extend $\nu$ to a valuation $\nu^{\prime}$ of $G$. Then by Proposition 4.5 there exists $\bar{\nu} \in \mathcal{V}(G)$ such that $\bar{\nu}(f)=\nu^{\prime}(g . f)$ for all $g$ in a nonempty open subset of $G$, and the claim follows because $\nu^{\prime}(g . f)=\nu(f)$ for all $f \in \mathrm{k}(G / H)$.

Let now $G / H$ be a spherical homogeneous space, and assume that $B H \subset G$ is open. Given a subspace $M \subset \mathrm{k}[G]$, let $M^{n}$ be the subspace generated by all the possible products of $n$ elements of $M$.

Proposition 4.7 ([17, Theorem 2.6]). Let $\nu \in \mathcal{V}(G / H)$, let $f \in \mathrm{k}(G / H)$ and let $f_{0} \in \mathrm{k}(G)^{(B \times H)}$. Suppose that $f f_{0} \in \mathrm{k}[G]$, and let $M \subset \mathrm{k}[G]$ be the $G$-submodule generated by $f f_{0}$. Then the following hold:

i) $M^{n} f_{0}^{-n} \subset \mathrm{k}(G / H)$, for all $n \in \mathbb{N}$.

ii) $\nu(f)=\min \left\{\frac{1}{n} \nu\left(f^{\prime} f_{0}^{-n}\right) \mid n \in \mathbb{N}, f^{\prime} \in\left(M^{n}\right)^{(B)}\right\}$.

Proof i) Let $\chi \in \mathcal{X}(H)$ be the weight of $f_{0}$ with respect to the right action of $H$. Since the action of $G$ commutes with the action of $H$, all elements of $M^{n}$ are $H$ eigenvectors of weight $n \chi$. Therefore $M^{n} f_{0}^{-n} \subset \mathrm{k}(G)^{H}$, and by the basic properties of homogeneous spaces the latter coincides with $\mathrm{k}(G / H)$.

ii) Let $\bar{\nu} \in \mathcal{V}(G)$ be a lifting of $\nu$, which exists by Corollary 4.6, and set $q=$ $\bar{\nu}\left(f f_{0}\right)$. Define $V(q)=\left\{f^{\prime} \in \mathrm{k}[G] \mid \bar{\nu}\left(f^{\prime}\right) \geqslant q\right\}$ : since $\bar{\nu}$ is a $G$-invariant valuation, $V(q)$ is a $G$-submodule of $\mathrm{k}[G]$. Since $f f_{0} \in V(q)$, by the very definition of invariant valuation we get $M^{n} \subset V(n q)$. Let $f^{\prime} \in M^{n}$ : then

$$
\bar{\nu}\left(f^{\prime}\right) \geqslant n q=n \bar{\nu}\left(f f_{0}\right)
$$

hence

$$
\nu(f)=\bar{\nu}(f) \leqslant \frac{1}{n} \bar{\nu}\left(f^{\prime} f_{0}^{-n}\right)=\frac{1}{n} \nu\left(f^{\prime} f_{0}^{-n}\right),
$$

where we used i) in the last equality.

To show that $\nu(f)$ is actually the minimum of such expressions, consider the integral graded k-algebra $R=\bigoplus_{i \geqslant 0} M^{i}$ with the inherited $G$-action, and let $X$ be the corresponding affine $G$-variety. If $r \in R$, we denote by $r_{i}$ its component in $M^{i}$. If $r \neq 0$, define

$$
\nu^{\prime}(r)=\min _{i \in \mathbb{N}}\left\{\bar{\nu}\left(r_{i}\right)-i q\right\} .
$$

One checks that $\nu^{\prime}$ defines a $G$-invariant valuation on (the normalization of) $X$, and by (4.1) we have $\nu_{\mid R}^{\prime} \geqslant 0$. Therefore $\nu^{\prime}$ has a center $Y \subset X$, corresponding to the $G$-stable homogeneous prime ideal $\mathfrak{p}=\left\{r \in R \mid \nu^{\prime}(r)>0\right\} \subset R$. Since $f f_{0} \in M$ and $\nu^{\prime}\left(f f_{0}\right)=0$, it follows that $M / M \cap \mathfrak{p} \neq 0$ is a nonzero finite dimensional $G$-submodule of k $[Y]$, therefore by the Lie-Kolchin theorem there exists a nonzero element $a \in(M / M \cap \mathfrak{p})^{(B)}$. Applying Corollary 2.6 to $X$ and $Y$, there exist $n \in \mathbb{N}$ and $f^{\prime} \in R^{(B)}$ such that $\left.f^{\prime}\right|_{Y}=a^{n}$. Since $\mathfrak{p}$ is homogeneous, we can assume that $f^{\prime} \in\left(M^{n}\right)^{(B)}$. Since $a \neq 0$, we have $f^{\prime} \in R \backslash \mathfrak{p}$, hence $\nu^{\prime}\left(f^{\prime}\right)=0$. Therefore $\bar{\nu}\left(f^{\prime}\right)=n q=n \nu(f)+\bar{\nu}\left(f_{0}^{n}\right)$ and we get $\nu(f)=\frac{1}{n} \nu\left(f^{\prime} f_{0}^{-n}\right)$, which shows the claim.

Corollary 4.8 ([17, Corollary 2.7]). Let $f \in \mathrm{k}[B H / H]$ and let $\nu_{0} \in \mathcal{V}(G / H)$. Then there exist $n \in \mathbb{N}$ and $f_{0} \in \mathrm{k}(G / H)^{(B)}$ such that

i) $\nu_{0}\left(f_{0}\right)=\nu_{0}\left(f^{n}\right)$,

ii) $\nu\left(f_{0}\right) \geqslant \nu\left(f^{n}\right)$ for all $\nu \in \mathcal{V}(G / H)$,

iii) $\nu_{D}\left(f_{0}\right) \geqslant \nu_{D}\left(f^{n}\right)$ for all $D \in \Delta(G / H)$.

Proof Up to replacing $G$ by a finite covering, we may assume that $\operatorname{Pic}(G)=0$ (see $[20$, Proposition 4.6]), which means that $\mathrm{k}[G]$ is a factorial ring (see [12, Proposition B.72]). In particular, every divisor $\delta$ on $G$ is the divisor of a function $h \in \mathrm{k}(G)$, and if moreover $\delta$ is stable under $B \times H$ then $h \in \mathrm{k}(G)^{(B \times H)}$. Let $\pi: G \rightarrow G / H$ be the projection, and denote by $\delta$ the $B$-stable part of $\operatorname{div}\left(f^{-1}\right)$, where $f^{-1}$ is regarded 
as a rational function on $G / H$. Let $h \in \mathrm{k}(G)^{(B \times H)}$ be such that $\pi^{*}(\delta)=\operatorname{div}(h)$ : then $f h \in \mathrm{k}[G]$, hence by Proposition 4.7 there exist $n \in \mathbb{N}$ and $f^{\prime} \in \mathrm{k}[G]^{(B)}$ with $f^{\prime} h^{-n} \in \mathrm{k}(G / H)$ such that $\nu_{0}\left(f^{\prime} h^{-n}\right)=\nu_{0}\left(f^{n}\right)$ and $\nu\left(f^{\prime} h^{-n}\right) \geqslant \nu\left(f^{n}\right)$ for all $\nu \in \mathcal{V}(G / H)$. If moreover $D \in \Delta(G / H)$ and $D^{\prime}$ is an irreducible component of $\pi^{-1}(D)$, then $\nu_{D}\left(f^{n}\right)=\nu_{D^{\prime}}\left(h^{-n}\right) \leqslant \nu_{D^{\prime}}\left(f^{\prime} h^{-n}\right)=\nu_{D}\left(f^{\prime} h^{-n}\right)$.

Thanks to the sequence (2.1), every valuation $\nu$ of $G / H$ induces a homomorphism $\hat{\rho}_{G / H}(\nu): \Lambda(G / H) \longrightarrow \mathbb{Q}$, defined by setting $\left\langle\hat{\rho}_{G / H}(\nu), \lambda\right\rangle=\nu(f)$ where $f \in$ $\mathrm{k}(G / H)^{(B)}$ is any eigenfunction of weight $\lambda$. Therefore we get a map

$$
\hat{\rho}_{G / H}:\{\text { valuations on } G / H\} \longrightarrow \mathcal{Q}(G / H)
$$

Corollary 4.9 ([17, Corollary 2.8], [24, Proposition 7.4]). The restriction of $\hat{\rho}_{G / H}$ to $\mathcal{V}(G / H)$ is injective.

Proof Let $B x_{0} \subset G / H$ be the open $B$-orbit, then $\mathrm{k}(G / H)=\mathrm{k}\left(B x_{0}\right)$, so that every valuation on $G / H$ is uniquely determined by its restriction to $\mathrm{k}\left[B x_{0}\right]$. Let $\nu, \nu^{\prime} \in$ $\mathcal{V}(G / H)$, suppose that $\nu \neq \nu^{\prime}$ and let $f \in \mathrm{k}\left[B x_{0}\right]$ be such that $\nu(f) \neq \nu^{\prime}(f)$. Assume that $\nu(f)<\nu^{\prime}(f)$ : then by Corollary 4.8 there exist $n \in \mathbb{N}$ and $F \in \mathrm{k}(G / H)^{(B)}$ such that $\nu(F)=\nu\left(f^{n}\right)$ and $\nu^{\prime}(F) \geqslant \nu^{\prime}\left(f^{n}\right)$. Therefore $\nu^{\prime}(F)>\nu\left(f^{n}\right)=\nu(F)$ and it follows $\hat{\rho}_{G / H}(\nu) \neq \hat{\rho}_{G / H}\left(\nu^{\prime}\right)$.

From now on, we will regard $\mathcal{V}(G / H)$ as a subset of $\mathcal{Q}(G / H)$ via the map $\hat{\rho}_{G / H}$.

\section{The $B$-Stable AfFine open subset associated to a $G$-Orbit}

Given a spherical variety $X$ and a $G$-orbit $Y \subset X$, in this section we show the existence of a canonical $B$-stable affine open subset of $X$ intersecting $Y$. This will be a main object in the classification of spherical embeddings. Our main tools will be Corollary 2.6 and Corollary 4.8.

Proposition 5.1 ([17, Theorem 2.3]). Let $X$ be a normal $G$-variety $X$ and let $Y \subset$ $X$ be a G-orbit. Then there exists a $B$-stable affine open subset $X_{0} \subset X$ intersecting $Y$ such that, for all $f \in \mathrm{k}\left[X_{0} \cap Y\right]^{(B)}$, there exist $n \in \mathbb{N}$ and $f^{\prime} \in \mathrm{k}\left[X_{0}\right]^{(B)}$ with $f_{\mid X_{0} \cap Y}^{\prime}=f^{n}$.

Proof By a theorem of Sumihiro (see [20, Theorem 1.1]) there exists a finite dimensional $G$-module $V$ and a $G$-stable open subset $U \subset X$ containing $Y$ such that $U$ embeds $G$-equivariantly in $\mathbb{P}(V)$. Therefore, up to replacing $X$ with $U$, we may assume that $X$ is equivariantly embedded in the projective space of a finite dimensional $G$-module $V$.

Let $\bar{X}$ be the closure of $X$ in $\mathbb{P}(V)$ and set $Z=\bar{X} \backslash X$. Let $I(Z)$ and $I(\bar{Y})$ be the homogeneous ideals in $\mathrm{k}[V]$ respectively of $Z$ and of $\bar{Y}$, the closure of $Y$ in $\mathbb{P}(V)$. Then $I(Z)$ and $I(\bar{Y})$ are $G$-stable, and since $Y \not \subset Z$ we have $I(Z) \not \subset I(\bar{Y})$. Let $f \in I(Z) \backslash I(\bar{Y})$ and let $M \subset I(Z)$ be the (finite dimensional) $G$-module generated by $f$ and denote $R=\bigoplus_{n \geqslant 0} M^{n}$. Then by the Lie-Kolchin theorem $M / M \cap I(\bar{Y})$ contains a nonzero $B$-eigenvector $v$. By Corollary 2.6 applied to $\operatorname{Spec}(R)$ and its subvariety defined by $I(\bar{Y})$, there exists $n \in \mathbb{N}$ such that $v^{n} \in R / R \cap I(\bar{Y})$ lifts to an element $f_{0} \in R^{(B)}$. Since the ideal $I(\bar{Y})$ is homogeneous, we can assume that $f_{0}$ is homogeneous, therefore we can define a $B$-stable affine open subset of $X$ by setting $X_{0}=\left\{x \in \bar{X} \mid f_{0}(x) \neq 0\right\}$. Since $v \in M / M \cap I(\bar{Y})$ is nonzero, notice that $f_{0} \in I(Z) \backslash I(\bar{Y})$, hence $X_{0} \cap Y \neq \varnothing$ and $X_{0} \subset X$.

Let now $f \in \mathrm{k}\left[X_{0} \cap Y\right]^{(B)}$. If $\hat{Y} \subset V$ is the affine cone over $Y$, let $d \in \mathbb{N}$ be such that $f_{0}^{d} f \in \mathrm{k}[\hat{Y}]$ is homogeneous. If $\hat{X}$ denotes the affine cone over $X$, then by Corollary 2.6 there exists $n \in \mathbb{N}$ such that $\left(f_{0}^{d} f\right)^{n}$ lifts to an element $f^{\prime \prime} \in \mathrm{k}[\hat{X}]^{(B)}$ which we can choose to be homogeneous, and we conclude by setting $f^{\prime}=f^{\prime \prime} f_{0}^{-d n}$. 
If $Y \subset X$ is a $G$-orbit, define

$$
X_{Y, B}=X \backslash \bigcup_{\mathcal{D}(X) \backslash \mathcal{D}_{Y}(X)} D \quad \text { and } \quad X_{Y, G}=G X_{Y, B}
$$

Theorem 5.2 ([17, Theorem 3.1]). Let $X$ be a spherical $G$-variety and let $Y \subset X$ be a G-orbit.

i) $X_{Y, B}$ is the minimal B-stable affine open subset of $X$ which intersects $Y$;

ii) $Y$ is the unique closed $G$-orbit in $X_{Y, G}$, and

$$
X_{Y, G}=\{x \in X \mid Y \subset \overline{G x}\} ;
$$

iii) $X_{Y, B} \cap Y$ is a single $B$-orbit.

Proof Let $X_{0}$ be a $B$-stable affine open subset intersecting $Y$ as in Proposition 5.1, and let $\nu_{0} \in \mathcal{V}(X)$ be a valuation with center $\bar{Y}$ (which exists by Remark 4.4). Let $f_{0} \in \mathrm{k}\left[X_{0}\right]$ be a function not vanishing on $\bar{Y} \cap X_{0}$ such that $\nu_{D}\left(f_{0}\right)>0$ for all $D \in \mathcal{D}(X) \backslash \mathcal{D}_{Y}(X)$ with $D \cap X_{0} \neq \varnothing$. Then $\nu_{0}\left(f_{0}\right)=0$, and by Corollary 4.8 we can assume that $f_{0} \in \mathrm{k}\left[X_{0}\right]^{(B)}$ : if $f_{0}^{\prime} \in \mathrm{k}(X)^{(B)}$ satisfies $\nu_{D}\left(f_{0}^{\prime}\right) \geqslant 0$ for all $D \in \mathcal{D}(X)$ with $D \cap X_{0} \neq \varnothing$, then $f_{0}^{\prime}$ is defined on all divisors of $X_{0}$, therefore $f_{0}^{\prime} \in \mathrm{k}\left[X_{0}\right]$ because $X$ is normal.

i) Clearly $X_{Y, B}$ is a $B$-stable open subset of $X$, and by [12, Exercise 12.18] it is contained inside every $B$-stable affine open subset which intersects $Y$. In particular, we have $X_{Y, B} \subset X_{0}$, and by the definition of $f_{0}$ we get $X_{Y, B}=\left\{x \in X_{0} \mid f_{0}(x) \neq\right.$ $0\}$.

ii) Let $Z \subset X$ be a $G$-orbit which intersects $X_{0}$ and suppose that $Y \not \subset \bar{Z}$. We may in addition assume that the function $f_{0}$ defining $X_{Y, B}$ vanishes on $Z$. Hence $X_{Y, B} \cap Z=\varnothing$ and $Y$ is the unique closed $G$-orbit in $X_{Y, G}$. Set $X_{Y, G}^{\prime}=\{x \in$ $X \mid Y \subset \overline{G x}\}$, then we have inclusions $X_{Y, B} \subset X_{Y, G} \subset X_{Y, G}^{\prime}$. Let $x \in X_{Y, G}^{\prime}$ and suppose by contradiction that $G x \cap X_{Y, B}=\varnothing$ : then $G x$ is contained in some $D \in \mathcal{D}(X) \backslash \mathcal{D}_{Y}(X)$, hence $Y \not \subset \overline{G x}$, a contradiction.

iii) The claim follows if we prove that every nonzero function $f \in \mathrm{k}\left[X_{Y, B} \cap Y\right]^{(B)}$ is invertible: indeed, if $Z \subsetneq X_{Y, B} \cap Y$ is a closed $B$-orbit and $I(Z) \subset \mathrm{k}\left[X_{Y, B} \cap Y\right]$ is the corresponding ideal, then by the Lie-Kolchin theorem $I(Z)^{(B)} \neq 0$, and any element therein is a non-invertible $B$-eigenfunction. Suppose that $f \in \mathrm{k}\left[X_{Y, B} \cap Y\right]^{(B)}$ is nonzero. For some $d \in \mathbb{N}$ we have $f_{0}^{d} f \in \mathrm{k}\left[X_{0} \cap Y\right]^{(B)}$, hence by the definition of $X_{0}$ there exist $n \in \mathbb{N}$ and $f^{\prime} \in \mathrm{k}\left[X_{0}\right]^{(B)}$ such that $f_{\mid X_{0} \cap Y}^{\prime}=\left(f_{0}^{d} f\right)^{n}$. It follows that $f_{\mid X_{Y, B}}^{\prime}$ is invertible: indeed $\nu_{D}\left(f^{\prime}\right)=0$ for all $D \in \mathcal{D}_{Y}(X)$ because $f_{\mid X_{0} \cap Y}^{\prime} \neq 0$, hence $f^{\prime}$ does not vanish on any divisor of $X_{Y, B}$. Therefore $f_{\mid X_{Y, B} \cap Y}^{\prime}$ is invertible, and $f$ is invertible as well.

Corollary 5.3 ([24, Proposition 7.5], [17, Corollary 3.2]). Let X be a multiplicityfree $G$-variety, then $X$ possesses finitely many $G$-orbits, and all of them are spherical.

Proof Up to replacing $X$ with its normalization, we may assume that $X$ is spherical. By Theorem 5.2 ii), every $G$-orbit $Y \subset X$ is uniquely determined by the corresponding $B$-stable affine open subset $X_{Y, B}$. On the other hand $\mathcal{D}(X)$ is a finite set by Proposition 2.20, hence there are finitely many possibilities for $X_{Y, B}$ and for $Y$ as well. This show the first claim, and the second one follows by Theorem $5.2 \mathrm{iii)}$.

Remark 5.4. Suppose that $\operatorname{char}(k)=0$. Then we have the equality

$$
X_{Y, B}=\{x \in X \mid Y \subset \overline{B x}\},
$$


namely $Y \cap X_{Y, B} \subset X_{Y, B}$ is the unique closed $B$-orbit. Indeed, denote $P=\{g \in$ $\left.G \mid g X_{Y, B}=X_{Y, B}\right\}$. Then $P$ is a parabolic subgroup of $G$, and if $P=L P_{\mathrm{u}}$ is the Levi decomposition of $P$ then by the local structure theorem (see e.g. [26, Theorem $3.2 .2])$ there exists a $L$-stable closed subvariety $S_{Y} \subset X_{Y, B}$ which is $L$-spherical and such that the $P$-action gives rise to a $P$-equivariant isomorphims $X_{Y, B} \simeq P \times{ }^{L} S_{Y}$. Since it is affine and it possesses an open $L$-orbit, we have $\mathrm{k}\left[S_{Y}\right]^{L}=\mathrm{k}$, thus the categorical quotient $S_{Y} / / L$ is a single point and there exists a unique closed $L$-orbit in $S_{Y}$. By Theorem 5.2, this $L$-orbit must be $S_{Y} \cap Y$ : indeed $Y$ is the unique closed $G$-orbit in $X_{Y, G}$, and $X_{Y, B} \cap Y$ is a single $B$-orbit. Let now $Z \subset X_{Y, B}$ be a closed $B$-orbit and let $x \in S_{Y}$ be such that $Z=B x$ : then $Z \cap S_{Y}=(B \cap L) x$ is a closed $(B \cap L)$-orbit in $S_{Y}$. It follows that $L x$ is a closed orbit in $S_{Y}$ : indeed, the $L$-action induces a morphism $L \times{ }^{B \cap L} S_{Y} \rightarrow S_{Y}$ which is proper because $L / B \cap L$ is projective (see e.g. [34, Proposition 2.7]), and $L x$ is the image of the closed subset $L \times{ }^{B \cap L}(B \cap L) x$. It follows that $L x=S_{Y} \cap Y$, and therefore $Z=X_{Y, B} \cap Y$.

Definition 5.5. A spherical $G$-variety $X$ is called simple if it contains a unique closed $G$-orbit. A spherical embedding $G / H \hookrightarrow X$ is called simple if $X$ is simple.

By Theorem 5.2 and Corollary 5.3, every spherical $G$-variety can be covered with finitely many simple spherical $G$-varieties, namely those of the shape $X_{Y, G}$, where $Y$ runs among the $G$-orbits of $X$.

Fix now a spherical $G$-variety $X$, let $B x_{0} \subset X$ be the open $B$-orbit and let $H \subset G$ be the stabilizer of $x_{0}$. Assume moreover that the orbit morphism $G \rightarrow G x_{0}$ is separable (e.g. if $\operatorname{char}(\mathrm{k})=0$ ). Then we can identify $G x_{0} \simeq G / H$, and we may regard $X$ as a spherical embedding of $G / H$. Since $G x_{0} \subset X_{Y, G}$ for all $G$-orbits $Y \subset X$, we can regard all the simple spherical varieties $X_{Y, G}$ as embeddings of the same homogeneous space $G / H$.

Every $G$-stable prime divisor $D \subset X$ is either $B$-stable (in which case $D \in \Delta(X)$ ) or $G$-stable (in which case $D \in \mathcal{D}(X) \backslash \Delta(X)$ ). Notice that we can canonically identify $\Delta(X)$ and $\Delta(G / H)$. On the other hand every $D \in \mathcal{D}(X) \backslash \Delta(X)$ defines a an invariant valuation $\nu_{D} \in \mathcal{V}(X)$, and if $Y \subset X$ is a $G$-orbit we can define a finite set of $G$-invariant valuations by setting

$$
\mathcal{V}_{Y}(X)=\left\{\nu_{D} \mid D \in \mathcal{D}_{Y}(X) \backslash \Delta(X)\right\} \subset \mathcal{V}(X)
$$

Since $\Delta(X)=\Delta(G / H), \Lambda(X)=\Lambda(G / H)$ and $\mathcal{V}(X)=\mathcal{V}(G / H)$ are all birational invariants of $X$, it follows that to every $G$-orbit $Y \subset X$ we may associate the following data on $G / H$ :

i) $\Delta_{Y}(X)$, a subset of the finite set $\Delta(G / H)$;

ii) $\mathcal{V}_{Y}(X)$, a finite subset of $\mathcal{V}(G / H)$.

These will be the main objects that will allow us to classify the spherical embeddings of $G / H$.

\section{Simple spherical EMBEDdings AND COlORED CONES}

Let $G / H$ be a spherical homogeneous space, we keep the notation of previous sections. In particular, we assume that $B H$ is open in $G$.

In this section, we will show that every simple spherical embedding $G / H \hookrightarrow X$ is uniquely determined by a strictly convex cone in $\mathcal{Q}(G / H)$ together with the set of colors $\Delta_{Y}(X) \subset \Delta(G / H)$. Conversely, we will also give a combinatorial characterization of the admissible pairs $(\mathcal{C}, \mathcal{F})$ with $\mathcal{C}$ a strictly convex cone in $\mathcal{Q}(G / H)$ and $\mathcal{F} \subset \Delta(G / H)$ which give rise to a simple spherical embedding of $G / H$, leading to the notion of colored cone. This generalizes the fact that in the theory of toric varieties (where colors do not exist) affine toric varieties with an effective action of a torus $T$ are classified by strictly convex cones in $\mathcal{X}(T)$. 
Let $\mathcal{C}_{Y}(X) \subset \mathcal{Q}(X)$ be the cone generated by $\rho\left(\Delta_{Y}(X)\right)$ together with $\mathcal{V}_{Y}(X)$.

Theorem 6.1 ([24, Proposition 8.3], [17, Theorem 3.3]). Let $G / H \hookrightarrow X$ be a simple spherical embedding with closed orbit $Y \subset X$. As a spherical embedding of $G / H, X$ is uniquely determined up to $G$-isomorphism by the pair $\left(\mathcal{C}_{Y}(X), \Delta_{Y}(X)\right)$.

Proof We first show that, among the spherical embeddings of $G / H, X$ is uniquely determined by the pair $\left(\mathcal{V}_{Y}(X), \Delta_{Y}(X)\right)$. Let

$$
U=G / H \cap X_{Y, B}=G / H \backslash \bigcup_{D \in \Delta(G / H) \backslash \Delta_{Y}(X)} D .
$$

By the algebraic version of Hartogs' theorem (see [12, Theorem 6.45]), regular functions on a normal variety always extend in codimension two. Therefore a function $f \in \mathrm{k}[U]$ extends to $X_{Y, B}$ if and only if $\nu(f) \geqslant 0$ for all $D \in \mathcal{D}_{Y}(X) \backslash \Delta(X)$, namely

$$
\mathrm{k}\left[X_{Y, B}\right]=\left\{f \in \mathrm{k}[U] \mid \nu(f) \geqslant 0 \quad \forall \nu \in \mathcal{V}_{Y}(X)\right\}
$$

Thus $X_{Y, B}$ is uniquely determined by $G / H$ together with $\left(\mathcal{V}_{Y}(X), \Delta_{Y}(X)\right)$.

Notice that, up to $G$-isomorphism, the spherical embedding $G / H \hookrightarrow X$ is uniquely determined by the pair $\left(\mathcal{V}_{Y}(X), \Delta_{Y}(X)\right)$. Indeed, let $G / H \hookrightarrow X^{\prime}$ be another simple spherical embedding with closed orbit $Y^{\prime} \subset X^{\prime}$, and suppose that $\left(\mathcal{V}_{Y^{\prime}}\left(X^{\prime}\right), \Delta_{Y^{\prime}}\left(X^{\prime}\right)\right)=\left(\mathcal{V}_{Y}(X), \Delta_{Y}(X)\right)$. Let $x_{0} \in X$ and $x_{0}^{\prime} \in X^{\prime}$ be the base points fixed by $H$, then by the previous discussion the $G$-equivariant birational morphism $\varphi: X \rightarrow X^{\prime}$ defined by the isomorphism $G x_{0} \rightarrow G x_{0}^{\prime}$ induces an isomorphism $X_{Y, B} \rightarrow X_{Y^{\prime}, B}^{\prime}$. Since $X$ is simple, Theorem 5.2 shows that $X=G X_{Y, B}$. Therefore $\varphi$ is defined everywhere, and since $X^{\prime}=G X_{Y^{\prime}, B}^{\prime}$ it follows that $\varphi$ is surjective. On the other hand $\varphi$ is a local isomorphism, therefore $X$ and $X^{\prime}$ are $G$-equivariantly isomorphic.

We now show that $\mathcal{V}_{Y}(X)$ can be recovered by the pair $\left(\mathcal{C}_{Y}(X), \Delta_{Y}(X)\right)$. Let $\nu_{0} \in \mathcal{V}_{Y}(X)$ and let $D_{0} \in \mathcal{D}_{Y}(X) \backslash \Delta(X)$ be the corresponding $G$-stable divisor. Let $f \in \mathrm{k}\left[X_{Y, B}\right]$ be a function vanishing on all $D \in \mathcal{D}_{Y}(X)$ except $D_{0}$. Then $\nu_{0}(f)=0$ and $\nu(f)>0$ for all $\nu \in \mathcal{V}_{Y}(X)$ different from $\nu_{0}$. By Corollary 4.8 there exists $f^{\prime} \in \mathrm{k}(G / H)^{(B)}$ with the same properties, and such that $\nu_{D}\left(f^{\prime}\right) \geqslant 0$ for all $D \in \Delta_{Y}(X)$. Therefore $\lambda_{f^{\prime}} \in \mathcal{C}_{Y}(X)^{\vee}$, and $\lambda_{f^{\prime}}^{\perp} \cap \mathcal{C}_{Y}(X)=\mathbb{Q}^{+} \nu_{0}$. Hence the half-lines $\mathbb{Q}^{+} \nu$ with $\nu \in \mathcal{V}_{Y}(X)$ are exactly the extremal rays of $\mathcal{C}_{Y}(X)$ which do not contain any element of $\rho\left(\Delta_{Y}(X)\right)$, and they are uniquely determined by the pair $\left(\mathcal{C}_{Y}(X), \Delta_{Y}(X)\right)$. To conclude the proof, it is enough to notice that every $\nu \in \mathcal{V}_{Y}(X)$ is uniquely determined by its ray $\mathbb{Q}^{+} \nu$ : indeed, since $\nu$ is the valuation defined by a divisor, its image is precisely $\mathbb{Z}$.

Proposition 6.2 ([17, Theorem 3.5]). Let $G / H \hookrightarrow X$ be a simple spherical embedding with closed orbit $Y \subset X$ and let $\nu \in \mathcal{V}(G / H)$. Then the following hold:

i) $\mathrm{k}\left[X_{Y, B}\right]^{(B)}=\left\{f \in \mathrm{k}(G / H)^{(B)} \mid \lambda_{f} \in \mathcal{C}_{Y}(X)^{\vee}\right\}$;

ii) $\nu$ has center on $X$ if and only if $\nu \in \mathcal{C}_{Y}(X)$;

iii) the center of $\nu$ is $Y$ if and only if $\nu \in \mathcal{C}_{Y}(X)^{\circ}$.

Proof i) Let $B x_{0} \subset X$ be the open $B$-orbit. By definition the $B$-stable prime divisors of $X_{Y, B}$ correspond to the elements of $\mathcal{D}_{Y}(X)$. Since $X_{Y, B}$ is normal, it follows that a function $f \in \mathrm{k}\left[B x_{0}\right]^{(B)}$ extends to $X_{Y, B}$ if and only if $\nu_{D}(f) \geqslant 0$ for all $D \in \mathcal{D}_{Y}(X)$. On the other hand every function in $\mathrm{k}(G / H)^{(B)}$ is regular when restricted to $B x_{0}$, therefore $f$ extends to $X_{Y, B}$ if and only if $\lambda_{f} \in \mathcal{C}_{Y}(X)^{\vee}$.

ii) Since $\nu$ is $G$-invariant and since $X=G X_{Y, B}$, it follows that $\nu$ has center on $X$ if and only if it has center on $X_{Y, B}$. By Proposition 4.3 the latter holds if and only if $\nu(f) \geqslant 0$ for all $f \in \mathrm{k}\left[X_{Y, B}\right]$. On the other hand by Corollary 4.8 for all 
$f \in \mathrm{k}\left[X_{Y, B}\right]$ there exist $f^{\prime} \in \mathrm{k}\left[X_{Y, B}\right]^{(B)}$ and $n \in \mathbb{N}$ such that $\nu\left(f^{\prime}\right)=\nu\left(f^{n}\right)$. It follows from i) that $\nu$ has center on $X$ if and only if $\nu \in \mathcal{C}_{Y}(X)$.

iii) Given $\nu \in \mathcal{V}(G / H) \cap \mathcal{C}_{Y}(X)$, notice that $\nu \in \mathcal{C}_{Y}(X)^{\circ}$ if and only if $\nu^{\perp} \cap$ $\mathcal{C}_{Y}(X)^{\vee}=\mathcal{C}_{Y}(X)^{\perp}$. Suppose that $Y$ is the center of $\nu$. Since $Y$ is contained in every $B$-stable divisor of $X_{Y, B}$, it follows that every function $f \in \mathrm{k}\left[X_{Y, B}\right]^{(B)}$ with $\nu(f)=0$ is invertible. Therefore $\nu^{\perp} \cap \mathcal{C}_{Y}(X)^{\vee}=\mathcal{C}_{Y}(X)^{\perp}$ by i), hence $\nu \in \mathcal{C}_{Y}(X)^{\circ}$.

Let now $\nu \in \mathcal{V}(G / H) \cap \mathcal{C}_{Y}(X)$ with center $Z$, and assume $Z \neq Y$. Let $f \in \mathrm{k}\left[X_{Y, B}\right]$ be a function vanishing on $Y$ and not on $Z$, by Corollary 4.8 we may assume that $f \in \mathrm{k}\left[X_{Y, B}\right]^{(B)}$. If $\nu_{0} \in \mathcal{V}(G / H)$ is a valuation with center $Y$, it follows that $\left\langle\nu, \lambda_{f}\right\rangle=0$ and $\left\langle\nu_{0}, \lambda_{f}\right\rangle>0$, therefore $\lambda_{f} \in \nu^{\perp} \backslash \mathcal{C}_{Y}(X)^{\perp}$ and $\nu \notin \mathcal{C}_{Y}(X)^{\circ}$.

We come now to the main definition of this section, and to the corresponding classification theorem.

Definition 6.3. A colored cone for $G / H$ is a pair $(\mathcal{C}, \mathcal{F})$ where $\mathcal{C} \subset \mathcal{Q}(G / H)$ and $\mathcal{F} \subset \Delta(G / H)$ satisfy the following conditions:

$(\mathrm{CC} 1) \mathcal{C}$ is a convex cone generated by $\rho(\mathcal{F})$ and by finitely many elements of $\mathcal{V}(G / H)$.

(CC2) The intersection $\mathcal{C}^{\circ} \cap \mathcal{V}(G / H)$ is nonempty.

A colored cone $(\mathcal{C}, \mathcal{F})$ is called strictly convex if the following condition holds:

(SCC) $\mathcal{C}$ is strictly convex, and $0 \notin \rho(\mathcal{F})$.

Proposition 6.4. Let $G / H \hookrightarrow X$ be a simple spherical embedding with closed orbit $Y \subset X$, then $\left(\mathcal{C}_{Y}(X), \Delta_{Y}(X)\right)$ is a strictly convex colored cone, and up to $G$ isomorphism $X$ is uniquely determined by such a pair among the simple spherical embeddings of $G / H$.

Proof To show that $\left(\mathcal{C}_{Y}(X), \Delta_{Y}(X)\right)$ is a colored cone, notice that (CC1) holds by definition, whereas $(\mathrm{CC} 2)$ holds by Remark 4.4 together with Proposition 6.2. To show (SCC), let $f \in \mathrm{k}\left[X_{Y, B}\right]$ be a function vanishing on every $B$-stable prime divisor of $X_{Y, B}$. By Corollary 4.8 we may assume $f \in \mathrm{k}\left[X_{Y, B}\right]^{(B)}$ : therefore $\nu_{D}(f)>0$ for all $D \in \mathcal{D}_{Y}(X)$, and $0 \notin \rho\left(\Delta_{Y}(X)\right)$. Moreover $\lambda_{f} \in \mathcal{C}_{Y}(X)^{\vee}$ and $\lambda_{f}^{\perp} \cap \mathcal{C}_{Y}(X)=0$, hence we get (SCC). The last claim was already proved in Theorem 6.1.

Theorem 6.5 ([24, Theorem 8.10], [17, Theorem 4.1]). Up to G-isomorphism, the map which associates to a simple spherical embedding $G / H \hookrightarrow X$ the corresponding colored cone is a bijection between the $G$-isomorphism classes of simple spherical embeddings of $G / H$ and the strictly convex colored cones for $G / H$.

The rest of the section will be devoted to the proof of previous theorem. We will split the proof in several partial results. For simplicity,we will denote $\Delta(G / H)$, $\Lambda(G / H), \mathcal{V}(G / H), \mathcal{Q}(G / H)$ and $\rho_{G / H}$ simply by $\Delta, \Lambda, \mathcal{V}, \mathcal{Q}$ and $\rho$.

We already showed the uniqueness part of the theorem. So we are left to show that every strictly convex colored cone corresponds to a simple spherical embedding of $G / H$. Let $(\mathcal{C}, \mathcal{F})$ be a strictly convex colored cone. By $(\mathrm{CC} 1)$ the cone $\mathcal{C}$ is finitely generated, therefore $\mathcal{C}^{\vee} \cap \Lambda$ is a finitely generated semigroup by Gordan's Lemma. Let $\lambda_{1}, \ldots, \lambda_{m} \in \mathcal{C}^{\vee} \cap \Lambda$ be a set of generators, and let $F_{1}, \ldots, F_{m} \in \mathrm{k}(G / H)^{(B)}$ with weights $\lambda_{1}, \ldots, \lambda_{m}$. Let $D_{0}$ be the union of the colors $D \in \Delta \backslash \mathcal{F}$, then the poles of $F_{1}, \ldots, F_{m}$ on $G / H$ are contained inside $D_{0}$. Let $\pi: G \rightarrow G / H$ be the projection, and let $f_{0} \in \mathrm{k}[G]^{(B \times H)}$ be a function vanishing precisely on $\pi^{-1}\left(D_{0}\right)$ and such that $f_{i}:=f_{0} F_{i} \in \mathrm{k}[G]$ for all $i \leqslant m$. Let $\chi \in \mathcal{X}(H)$ be the weight of $f_{0}$ with respect to the right action of $H$, and notice that $f_{i} \in \mathrm{k}[G]_{\chi}^{(H)}$ for all $i \leqslant m$.

Let $V \subset \mathrm{k}[G]$ be the $G$-module generated by $f_{0}, \ldots, f_{m}$, then the evaluation of functions $(g, f) \mapsto f\left(g^{-1}\right)$ defines an equivariant morphism $G \rightarrow V^{*}$. Since $V$ is $G$-stable, the image of $G$ does not contain 0: indeed if $g^{\prime} \in G$ is such that $f\left(g^{\prime}\right)=0$ 
for all $f \in V$, then $f(g)=\left(g^{\prime} g^{-1} \cdot f\right)\left(g^{\prime}\right)=0$ for all $g \in G$, hence $f=0$. Since $V \subset \mathrm{k}[G]_{\chi}^{(H)}$, we get then an equivariant morphism $\varphi: G / H \rightarrow \mathbb{P}\left(V^{*}\right)$. Let

$$
X_{0}^{\prime}=\left\{x \in \overline{\varphi(G / H)} \mid f_{0}(x) \neq 0\right\}
$$

and define $X^{\prime}=G X_{0}^{\prime}$. Notice that $X^{\prime}$ is a multiplicity-free $G$-variety (because $G / H$ is so) and that $X_{0}^{\prime}$ is a $B$-stable affine open subset in $X^{\prime}$ containing the open $B$-orbit $B x_{0}^{\prime}$, where $x_{0} \in X^{\prime}$ denotes the image of the base point of $G / H$.

Lemma 6.6. In the above notation, the following equality holds:

$$
\mathrm{k}\left[X_{0}^{\prime}\right]^{(B)}=\left\{f \in \mathrm{k}(G / H)^{(B)} \mid \lambda_{f} \in \mathcal{C}^{\vee}\right\} .
$$

In particular, if $\Upsilon \subset \Lambda$ is the semigroup of the weights of $B$ occurring in $\mathrm{k}\left[X_{0}^{\prime}\right]^{(B)}$, then $\mathcal{C}=\operatorname{cone}(\Upsilon)^{\vee}$.

Proof Observe first of all that $\mathrm{k}\left[X_{0}^{\prime}\right]$ is the subalgebra of $\mathrm{k}(G / H)$ generated by the functions $f^{\prime} / f_{0}$ with $f^{\prime} \in V$. Let $f \in \mathrm{k}(G / H)^{(B)}$ and suppose that $\lambda_{f} \in \mathcal{C}^{\vee}$, then by construction $\lambda_{f}=\sum a_{i} \lambda_{i}$ is a linear combination with integral coefficients of $\lambda_{1}, \ldots, \lambda_{m}$. Therefore the function

$$
\left(f_{1} / f_{0}\right)^{a_{1}} \cdots\left(f_{m} / f_{0}\right)^{a_{m}} \in \mathrm{k}\left[X_{0}^{\prime}\right]^{(B)}
$$

has weight $\lambda_{f}$, hence up to a scalar factor this function is precisely $f$.

Conversely, let $f \in \mathrm{k}\left[X_{0}^{\prime}\right]^{(B)}$ and let $\nu \in \mathcal{C}$ be an element generating an extremal ray. By $(\mathrm{CC} 1)$, up to rescaling we may assume that $\nu \in \rho(\mathcal{F}) \cup \mathcal{V}$. Since the poles of $f$ are all contained in $D_{0}$, it follows that $\nu(f) \geqslant 0$ if $\nu \in \rho(\mathcal{F})$. Suppose that $\nu \in \mathcal{C} \cap \mathcal{V}$ and notice that by construction we have $\nu(f) \geqslant 0$ if $f=\frac{f_{i}}{f_{0}}=F_{i}$ for some $i$ with $1 \leqslant i \leqslant m$. For a general $f$, write $f=f^{\prime} / f_{0}^{n}$ with $f^{\prime} \in \mathrm{S}^{n} V$, and extend $\nu$ to an invariant valuation $\bar{\nu} \in \mathcal{V}(G)$ as in Corollary 4.6. Write $f^{\prime}=\prod_{i=1}^{n} \sum_{j} a_{i j} g_{i j} . f_{k_{i j}}$ with $a_{i j} \in \mathrm{k}$ and $g_{i j} \in G$ for some choice of the indices $0 \leqslant k_{i j} \leqslant m$, then we get

$$
\nu(f) \geqslant \sum_{i=1}^{n} \min _{j}\left\{\bar{\nu}\left(f_{k_{i j}}\right)-\bar{\nu}\left(f_{0}\right)\right\} \geqslant \min \left\{\nu(1), \nu\left(F_{1}\right), \ldots, \nu\left(F_{m}\right)\right\} \geqslant 0 .
$$

Lemma 6.7. The morphism $\varphi: G / H \rightarrow X^{\prime}$ has finite fibers.

Proof We show that the fibers of $\varphi$ are both affine and complete.

To show that the fibers of $\varphi$ are affine, it is enough to show that $\varphi^{-1}\left(B x_{0}^{\prime}\right)=$ $B H / H$ : indeed $B H / H$ is an affine variety by Theorem 2.17 , and we can cover $G / H$ by its translates. If $D \in \Delta \backslash \mathcal{F}$, then by construction $f_{0}$ vanishes on $\pi^{-1}(D)$, hence $\varphi(D) \subset X^{\prime} \backslash X_{0}$, and in particular $\varphi(D) \cap B x_{0}^{\prime}=\varnothing$. If $D \in \mathcal{F}$, then $\rho(D)^{\perp} \cap \mathcal{C}^{\vee}$ is a proper face of $\mathcal{C}^{\vee}$ by (SCC). Hence there exists $\lambda \in \mathcal{C}^{\vee} \cap \Lambda$ such that $\langle\rho(D), \lambda\rangle>0$, and by Lemma 6.6 there exists a nonzero function $h \in \mathrm{k}\left[X_{0}^{\prime}\right]^{(B)}$ vanishing on $D$. Therefore $\varphi(D) \cap B x_{0}^{\prime}=\varnothing$ for all $D \in \Delta$. On the other hand by Proposition $2.20 \Delta$ is the set of irreducible components of $G / H \backslash B H / H$, therefore we get $\varphi^{-1}\left(B x_{0}^{\prime}\right)=B H / H$.

Let now $X^{\prime \prime}$ be any complete embedding of $G / H$ (which exists for instance by Chevalley's theorem [31, Theorem 5.5.3]). Let $X^{\prime \prime \prime}$ be the normalization of the closure of $G / H$ diagonally embedded in $X^{\prime} \times X^{\prime \prime}$. Then the projection $\bar{\varphi}: X^{\prime \prime \prime} \rightarrow X^{\prime}$ is a proper morphism which extends $\varphi$, and to show that the fibers of $\varphi$ are complete it is enough to show that $\bar{\varphi}^{-1}\left(G x_{0}^{\prime}\right)=G / H$. Suppose that $Z \subset X^{\prime \prime \prime} \backslash G / H$ is a $G$-stable subvariety mapping dominantly on $X^{\prime}$, by Remark 4.4 there exists a valuation $\nu \in \mathcal{V}$ having center $Z$ on $X^{\prime \prime \prime}$. Then $\nu$ vanishes identically on $\mathrm{k}\left(X^{\prime}\right)$, hence on its subset $\mathrm{k}\left[X_{0}^{\prime}\right]^{(B)}$. On the other hand $\mathcal{C}$ is strictly convex by (SCC), hence $\mathcal{C}^{\vee}$ has maximal dimension and $\Lambda$ is generated by $\mathcal{C}^{\vee} \cap \Lambda$. It follows that $\mathrm{k}(G / H)^{(B)}$ is generated as a multiplicative group by $\mathrm{k}\left[X_{0}\right]^{(B)}$, therefore $\nu(f)=0$ 
for all $f \in \mathrm{k}(G / H)^{(B)}$. Since $\nu$ is not the trivial valuation, we get a contradiction by Corollary 4.9 .

Since $\varphi$ has finite fibers, it follows by a version of Zariski's main theorem (see [12, Corollary 12.87]) that there exists a variety $X$ such that $\varphi$ factors through an open embedding $\iota: G / H \hookrightarrow X$ and a finite morphism $\psi: X \rightarrow X^{\prime}$. Since $G / H$ is normal, we can take $X$ to be the normalization of $X^{\prime}$ in $\mathrm{k}(G / H)$ (see [12, Section 12.11] for the definition of the latter). In particular, considering the morphism given by the $G$-actions $\alpha: G \times G / H \rightarrow G / H$ and $\beta^{\prime}: G \times X^{\prime} \rightarrow X^{\prime}$, by the universal property of the normalization we get a canonical morphism $\beta: G \times X \rightarrow X$ which makes the following diagram commute:

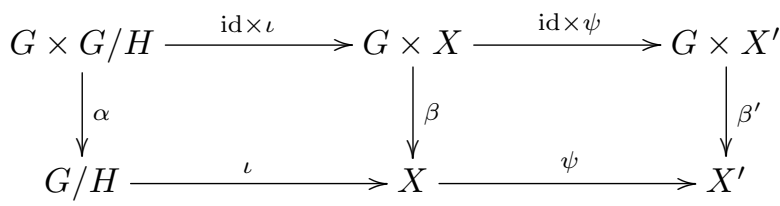

Notice that $\beta$ makes $X$ into a $G$-variety, in such a way that $\iota$ and $\psi$ are $G$-equivariant morphisms. In particular, we get a spherical embedding $G / H \hookrightarrow X$.

Denote $X_{0}=\psi^{-1}\left(X_{0}^{\prime}\right)$, since $\psi$ is a finite morphism $X_{0}$ is a $B$-stable affine open subset of $X$, which contains the open $B$-orbit $B x_{0}$, where $x_{0} \in X$ denotes the image of the base point of $G / H$. Since $\psi$ induces an inclusion $\mathrm{k}\left[X_{0}^{\prime}\right] \subset \mathrm{k}\left[X_{0}\right]$, for all $B$-stable prime divisors $D \subset X_{0}$ and for all $f \in \mathrm{k}\left[X_{0}^{\prime}\right]^{(B)}$ we have $\nu_{D}(f) \geqslant 0$. In particular by Lemma 6.6 it follows that $\rho\left(\nu_{D}\right) \in \operatorname{cone}(\Upsilon)^{\vee}=\mathcal{C}$ for all $D \in \mathcal{D}(X)$ such that $D \cap X_{0} \neq \varnothing$. Since $X_{0}$ is normal and $\mathrm{k}(G / H)^{(B)}=\mathrm{k}\left[B x_{0}\right]^{(B)}$,

$$
\mathrm{k}\left[X_{0}\right]^{(B)}=\left\{f \in \mathrm{k}(G / H)^{(B)} \mid \nu_{D}(f) \geqslant 0 \text { for all } B \text {-stable prime divisor of } X_{0}\right\},
$$

therefore $\mathrm{k}\left[X_{0}\right]^{(B)} \subset\left\{f \in \mathrm{k}(G / H)^{(B)} \mid \lambda_{f} \in \mathcal{C}^{\vee}\right\}$, and again by Lemma 6.6 we get the equality $\mathrm{k}\left[X_{0}\right]^{(B)}=\mathrm{k}\left[X_{0}^{\prime}\right]^{(B)}$.

Proposition 6.8. The morphism $G / H \hookrightarrow X$ is a simple embedding, and if $Y$ is the closed $G$-orbit of $X$ then $X_{Y, B}=X_{0}$ and $\left(\mathcal{C}_{Y}(X), \Delta_{Y}(X)\right)=(\mathcal{C}, \mathcal{F})$.

Proof By (CC2), there exists an element $\nu_{0} \in \mathcal{V} \cap \mathcal{C}^{\circ}$. Thus $\nu_{0}$ is nonnegative on $\mathrm{k}\left[X_{0}\right]^{(B)}$ by the previous discussion, hence it is nonnegative on $\mathrm{k}\left[X_{0}\right]$ by Corollary 4.8. By Proposition 4.3 it follows that $\nu_{0}$ has a center on $X_{0}$, whose closure $Y \subset X$ is $G$-stable since $\nu_{0}$ is $G$-invariant.

Let $Z \subset X$ be a $G$-stable closed subvariety, and let $\nu \in \mathcal{V}$ be a valuation with center $Z$. By the construction of $X^{\prime}$ and $X$, we have $Z \cap X_{0} \neq \varnothing$. Therefore $\nu$ has a center on $X_{0}$ and it follows $\nu_{\mid \mathrm{k}\left[X_{0}\right]} \geqslant 0$, hence $\nu \in \mathcal{C}$. Suppose that $Y \not \subset Z$ and let $f \in \mathrm{k}\left[X_{0}\right]$ be a function which vanishes on $Z$ and not on $Y$, so that $\nu_{0}(f)=0$ and $\nu(f)>0$. On the other hand by Corollary 4.8 we may assume that $f \in \mathrm{k}\left[X_{0}\right]^{(B)}$, therefore $\left\langle\nu_{0}, \lambda_{f}\right\rangle=0$. Since $\nu_{0} \in \mathcal{C}^{\circ}$, it follows that $\lambda_{f}=0$, a contradicition since $\left\langle\nu, \lambda_{f}\right\rangle>0$. Therefore $Y \subset Z$ and $G / H \hookrightarrow X$ is a simple spherical embedding, with closed orbit $Y$.

Since $\rho(\mathcal{F}) \subset \mathcal{C}$, notice that the same argument works if $\nu=\nu_{D}$ for some $D \in \mathcal{F}$ : therefore $\mathcal{F} \subset \Delta_{Y}(X)$. The same argument also shows that $Y$ is contained in all $B$-stable prime divisors of $X_{0}$, which gives us that $X_{0} \subset X_{Y, B}$, thus $X_{0}=X_{Y, B}$ by the minimality of $X_{Y, B}$ in Theorem 5.2. By Proposition 6.2 we get then $\mathcal{C}^{\vee} \cap \Lambda=$ $\mathcal{C}_{Y}(X)^{\vee} \cap \Lambda$, thus $\mathcal{C}=\mathcal{C}_{Y}(X)$. Finally, by construction every $D \in \Delta \backslash \mathcal{F}$ maps on $\psi(G / H) \backslash X_{0}^{\prime}$, hence $\mathcal{F}=\Delta_{Y}(X)$.

Remark 6.9. If $\operatorname{char}(\mathrm{k})=0$, by making use of the local structure theorems (see [26, Proposition 3.1.1 and Theorem 3.2.2]) it is possible to show that, in the previous notation, $X \simeq X^{\prime}$. Indeed if $g \in G$, then $g \cdot X_{0}=X_{0}$ if and only if $g \cdot X_{0}^{\prime}=X_{0}^{\prime}$, 
therefore we can write $X \simeq P \times{ }^{L} S_{Y}$ and $X^{\prime} \simeq P \times{ }^{L} S_{Y^{\prime}}^{\prime}$, where $Y^{\prime}=\psi(Y)$ and $P=\left\{g \in G \mid g \cdot X_{0}=X_{0}\right\}$ and where $S_{Y} \subset X_{0}$ and $S_{Y^{\prime}}^{\prime} \subset X_{0}^{\prime}$ are suitable closed $L$-stable subvarieties, which are multiplicity-free. Moreover, $\psi$ restricts to a $L$-equivariant finite morphism $S_{Y} \rightarrow S_{Y^{\prime}}^{\prime}$, and $\Lambda^{+}\left(S_{Y^{\prime}}^{\prime}\right)=\Lambda \cap \mathcal{C}^{\vee}=\Lambda^{+}\left(S_{Y^{\prime}}^{\prime}\right)$. Therefore, by decomposing the coordinate rings into irreducible $L$-modules, it follows that the inclusion $\mathrm{k}\left[S_{Y^{\prime}}^{\prime}\right] \subset \mathrm{k}\left[S_{Y}\right]$ is actually an equality. Therefore $S_{Y^{\prime}}^{\prime} \simeq S_{Y}$ are $L$-equivariantly isomorphic, hence $X_{0} \simeq X_{0}^{\prime}$ are isomorphic $P$-varieties, and $X \simeq X^{\prime}$ are isomorphic $G$-varieties.

Example 6.10. Regard $\mathbb{A}^{2}$ as a $\mathrm{SL}_{2}$-variety, then $\mathbb{A}^{2} \backslash\{0\}$ is an open orbit, and the stabilizer of $(1,0)$ is the maximal unipotent subgroup $U$ of unipotent upper triangular matrices. Therefore $\mathrm{SL}_{2} / U \hookrightarrow \mathbb{A}^{2}$ is a spherical embedding. Let $B$ the Borel subgroup of upper triangular matrices. Notice that there are three $B$-orbits in $\mathbb{A}^{2}$ : the fixed point 0 , the codimension one $B$-orbit $D=\{(a, 0) \mid a \neq 0\}$ and the open $B$-orbit $\{(a, b) \mid b \neq 0\}$. It follows that $\Delta_{0}\left(\mathbb{A}^{2}\right)=\Delta\left(\mathrm{SL}_{2} / U\right)=\{D\}$ and $\mathbb{A}_{0, B}^{2}=\mathbb{A}^{2}$, therefore by Proposition 6.2 it follows that $\mathcal{C}_{0}\left(\mathbb{A}^{2}\right)=\operatorname{cone}\left(\Lambda^{+}\left(\mathbb{A}^{2}\right)\right)^{\vee}$. On the other hand $\mathrm{k}[x, y]^{(B)}=\left\{y^{n} \mid n \in \mathbb{N}\right\}$, therefore $\Lambda^{+}\left(\mathbb{A}^{2}\right)=\Lambda^{+}$and $\mathcal{C}_{0}\left(\mathbb{A}^{2}\right) \subset$ $\mathcal{Q}=\Lambda_{\mathbb{Q}} \simeq \mathbb{Q}$ is the positive half-line generated by the simple coroot $\alpha^{\vee}$. Notice that $\rho(D)=\alpha^{\vee}$. As for all horospherical varieties, we will see in Corollary 11.7 that in this case $\mathcal{V}=\mathcal{Q}$.

Regard now $\mathbb{A}^{2}$ as a $\mathbb{G}_{m}^{2}$-variety, then there are four orbits: the open orbit $\{(a, b) \mid a \neq 0, b \neq 0\}$, two codimension one orbits $\{(a, 0) \mid a \neq 0\}$ and $\{(0, b) \mid b \neq$ $0\}$, and the fixed point 0 . Again every $\mathbb{G}_{m}^{2}$-stable divisor contains the origin, hence $\mathbb{A}_{0, B}^{2}=\mathbb{A}^{2}$ and $\mathcal{C}_{0}\left(\mathbb{A}^{2}\right)=\operatorname{cone}\left(\Lambda^{+}\left(\mathbb{A}^{2}\right)\right)^{\vee}$. On the other hand $\mathrm{k}[x, y]^{\left(\mathbb{G}_{m}^{2}\right)}=$ $\left\{x^{m} y^{n} \mid m, n \in \mathbb{N}\right\}$, therefore $\mathcal{C}_{0}\left(\mathbb{A}^{2}\right)$ is the positive quadrant in $\mathcal{Q} \simeq \mathbb{Q}^{2}$.

Example 6.11 (Symmetric matrices, part III). Consider the space $X=\mathrm{Sym}_{n}$ of symmetric matrices with the action of $G=\mathrm{GL}_{n}$ as in Example 3.4, and keep the notation therein. Let $Y \subset X$ be the unique closed $G$-orbit (namely the fixed point $\left.x_{0}=0\right)$. Since $x_{0} \in D_{i}$ for all $i \leqslant n$, it follows that $\Delta_{Y}(X)=\Delta(X)$ and $X_{Y, B}=X$. Therefore by Proposition 6.2 we get

$$
\mathcal{C}_{Y}(X)=\operatorname{cone}\left(\frac{1}{2} \alpha_{1}^{\vee}, \ldots, \frac{1}{2} \alpha_{n-1}^{\vee}, \frac{1}{2} \omega_{n}^{*}\right) .
$$

Example 6.12 (The space of quadrics). Keep the notation of Example 3.4 and consider now the space of quadrics $Q_{n}=\mathbb{P}\left(\mathrm{Sym}_{n}\right)$, which is spherical under the action of $\bar{G}=\mathrm{PGL}_{n}$ with open orbit $\mathrm{PGL}_{n} / \mathrm{PSO}_{n}$. Denote by $\bar{B}$ (resp. by $\bar{U}, \bar{T}$ ) the image of $B$ (resp. of $U, T$ ) in $\bar{G}$, and for a subvariety $Z \subset \operatorname{Sym}_{n}$ denote by $\bar{Z}$ its image in $Q_{n}$. Then the $\bar{G}$-orbits in $Q_{n}$ are the images of the nonzero $G$-orbits in $\operatorname{Sym}_{n}$, thus $\bar{X}_{n-1}$ is the unique $\bar{G}$-stable divisor and $Y=\bar{X}_{1}$ is the unique closed $\bar{G}$-orbit. Similarly, $\mathcal{D}\left(Q_{n}\right)=\left\{\bar{D}_{1}, \ldots, \bar{D}_{n}\right\}$ and $\Delta\left(Q_{n}\right)=\left\{\bar{D}_{1}, \ldots \bar{D}_{n-1}\right\}$, hence $\Delta_{Y}\left(Q_{n}\right)=\Delta\left(Q_{n}\right) \backslash\left\{\bar{D}_{1}\right\}$. Therefore the $B$-stable affine open subset of $Q_{n}$ associated to $Y$ is $Q_{n}^{0}=Q_{n} \cap\left\{d_{1} \neq 0\right\}$ and

$$
\mathrm{k}\left[Q_{n}^{0}\right]^{\bar{U}}=\left\{f / d_{1}^{m} \mid f \in \mathrm{k}\left[\operatorname{Sym}_{n}\right]^{U} \text { has degree } m\right\} .
$$

On the other hand $\mathrm{k}\left[\mathrm{Sym}_{n}\right]^{U}=\mathrm{k}\left[d_{1}, \ldots, d_{n}\right]$, and $d_{i}$ is a $T$-eigenfunction of degree $i$ of weight $2 \omega_{i}$. Therefore $\mathrm{k}\left[Q_{n}^{0}\right]^{(\bar{B})}$ is the set of monomials in the polynomial ring $\mathrm{k}\left[\frac{d_{2}}{d_{1}^{2}}, \ldots, \frac{d_{n}}{d_{1}^{n}}\right]$.

Notice that for $1<i \leqslant n$ the $T$-weight of $d_{i} d_{1}^{-i}$ is

$$
\lambda_{d_{i}}-i \lambda_{d_{1}}=2 \omega_{i}-2 i \omega_{1}=-\left((2 i-2) \alpha_{1}+(2 i-4) \alpha_{2}+\ldots+2 \alpha_{i-1}\right) .
$$

In particular, $\Lambda\left(Q_{n}\right)=2 \mathcal{X}(\bar{T})$. Let $\varpi_{1}, \ldots, \varpi_{n-1}$ be the fundamental weights of $\mathrm{PGL}_{n}$ (namely $\varpi_{i}=\omega_{i}-\frac{i}{n} \omega_{n}$, where $\omega_{1}, \ldots, \omega_{n}$ are the fundamental weights of $\left.\mathrm{GL}_{n}\right)$. As in Example 3.4, notice that $\nu_{\bar{D}_{i}}\left(d_{j} d_{1}^{-j}\right)=\delta_{i j}$ for all $i>1$. Therefore 
$\left\{\rho\left(\bar{D}_{2}\right), \ldots, \rho\left(\bar{D}_{n-1}\right), \nu_{\bar{D}_{n}}\right\}$ is the dual basis of $\left\{2 \omega_{i}-2 i \omega_{1} \mid i=2, \ldots, n\right\}$, and it follows $\rho\left(\bar{D}_{i}\right)=\frac{1}{2} \alpha_{i}^{\vee}$ if $2 \leqslant i<n$ and $\nu_{\bar{D}_{n}}=-\frac{1}{2} \varpi_{n-1}^{\vee}\left(\right.$ where $\varpi_{1}^{\vee}, \ldots, \varpi_{n-1}^{\vee}$ are the fundamental coweights). Thus we get

$$
\mathcal{C}_{Y}\left(Q_{n}\right)=\operatorname{cone}\left(\frac{1}{2} \alpha_{2}^{\vee}, \ldots, \frac{1}{2} \alpha_{n-1}^{\vee},-\frac{1}{2} \varpi_{n-1}^{\vee}\right) .
$$

One can see that in this case $\mathcal{V}\left(Q_{n}\right)=\operatorname{cone}\left(-\frac{1}{2} \varpi_{1}^{\vee}, \ldots,-\frac{1}{2} \varpi_{n-1}^{\vee}\right)$ (see e.g. [35, Proposition 2], where the general case of a symmetric variety is considered). Therefore $\mathcal{V}\left(Q_{n}\right) \subset \mathcal{C}_{Y}\left(Q_{n}\right)$, and $\left(\mathcal{C}_{Y}\left(Q_{n}\right), \Delta_{Y}\left(Q_{n}\right)\right)$ is indeed a colored cone.

Remark 6.13. As already mentioned, the theory of spherical embeddings can be adapted (and possibly simplified) in several situations which are interesting in their own right. In particular, for the case of toric varieties we refer the reader to Fulton's book [10], for the case of symmetric spaces to Vust's paper [35], for the case of group embeddings to Timashev's paper [33].

\section{THE CLASSIFICATION OF SPHERICAL EMBEDDINGS}

We keep the notation of the previous sections. In particular, $G / H$ is a spherical homogeneous space, and $B H$ is open in $G$.

Definition 7.1. Let $(\mathcal{C}, \mathcal{F})$ be a colored cone. A face of $(\mathcal{C}, \mathcal{F})$ is a colored cone $\left(\mathcal{C}_{0}, \mathcal{F}_{0}\right)$ such that $\mathcal{C}_{0}$ is a face of $\mathcal{C}$ and $\mathcal{F}_{0}=\mathcal{F} \cap \rho^{-1}\left(\mathcal{C}_{0}\right)$.

Notice that a face of a colored cone $(\mathcal{C}, \mathcal{F})$ is uniquely determined by the corresponding face of $\mathcal{C}$ : the faces of $(\mathcal{C}, \mathcal{F})$ correspond to the faces of $\mathcal{C}$ which intersect $\mathcal{V}(G / H)$ nontrivially in their relative interior. In particular, we can regard every face of $(\mathcal{C}, \mathcal{F})$ as a face of $\mathcal{C}$.

Proposition 7.2 ([17, Lemma 4.2]). Let $G / H \hookrightarrow X$ be a spherical embedding and let $Y \subset X$ be a $G$-orbit. Then the map $Z \mapsto\left(\mathcal{C}_{Z}(X), \Delta_{Z}(X)\right)$ is a bijection between $G$-orbits in $X$ whose closure contain $Y$ and faces of $\left(\mathcal{C}_{Y}(X), \Delta_{Y}(X)\right)$.

Proof We may assume that $X$ is simple with closed orbit $Y$. Let $Z \subset X$ be a $G$-orbit, and let $f \in \mathrm{k}\left[X_{Y, B}\right]$ be a function vanishing on all $D \in \mathcal{D}_{Y}(X) \backslash \mathcal{D}_{Z}(X)$ and not vanishing on $Z$, then $\nu_{D}(f)=0$ for all $D \in \mathcal{D}_{Z}(X)$ and $\nu_{D}(f)>0$ for all $D \in \mathcal{D}_{Y}(X) \backslash \mathcal{D}_{Z}(X)$. Moreover, by Corollary 4.8 we may assume $f \in$ $\mathrm{k}\left[X_{Y, B}\right]^{(B)}$, therefore $\mathcal{C}_{Z}(X)$ is the face of $\mathcal{C}_{Y}(X)$ defined by $\lambda_{f}$. As well, it follows that $\Delta_{Z}(X)=\Delta_{Y}(X) \cap \rho^{-1}\left(\mathcal{C}_{Z}(X)\right)$, therefore $\left(\mathcal{C}_{Z}(X), \Delta_{Z}(X)\right)$ is a face of $\left(\mathcal{C}_{Y}(X), \Delta_{Y}(X)\right)$.

Let now $(\mathcal{C}, \mathcal{F})$ be a face of $\left(\mathcal{C}_{Y}(X), \Delta_{Y}(X)\right)$ and let $\nu \in \mathcal{V}(G / H) \cap \mathcal{C}^{\circ}$. By Proposition 6.2 it follows that $\nu$ has a center on $X$, which must be the closure of a $G$-orbit $Z$ because it is $G$-stable and there are finitely many orbits by Corollary 5.3. We already showed that $\left(\mathcal{C}_{Z}(X), \Delta_{Z}(X)\right)$ is a face of $\left(\mathcal{C}_{Y}(X), \Delta_{Y}(X)\right)$. Since $\nu$ has center $Z$, Proposition 6.2 implies that $\nu \in \mathcal{C}_{Z}(X)^{\circ}$ : therefore $\mathcal{C}=\mathcal{C}_{Z}(X)$ and $\mathcal{F}=\Delta_{Z}(X)$.

In particular, the poset of orbit closures of a simple spherical variety $X$ with closed orbit $Y$ is isomorphic to the poset of faces of $\mathcal{C}_{Y}(X)$ which intersect $\mathcal{V}(G / H)$ nontrivially in their relative interior, with the reverse order.

Definition 7.3. A colored fan is a nonempty set $\mathfrak{F}$ of colored cones with the following properties:

(CF1) Every face of a colored cone in $\mathfrak{F}$ belongs to $\mathfrak{F}$.

(CF2) For all $\nu \in \mathcal{V}(G / H)$ there is at most one colored cone $(\mathcal{C}, \mathcal{F}) \in \mathfrak{F}$ such that $\nu \in \mathcal{C}^{\circ}$.

A colored fan $\mathfrak{F}$ is strictly convex if all its elements are strictly convex, namely if $(0, \varnothing) \in \mathfrak{F}$. 
Given a spherical embedding $G / H \hookrightarrow X$, we denote

$$
\mathfrak{F}(X)=\left\{\left(\mathcal{C}_{Y}(X), \Delta_{Y}(X)\right): Y \subset X \text { is a } G \text {-orbit }\right\} .
$$

Theorem 7.4 ([17, Theorem 4.3]). Up to G-isomorphism, the map which associates $\mathfrak{F}(X)$ to $G / H \hookrightarrow X$ is a bijection between spherical embeddings of $G / H$ and strictly convex colored fans.

Proof Let $G / H \hookrightarrow X$ be a spherical embedding. Then (CF1) holds by Proposition 7.2 , and $\mathfrak{F}(X)$ is strictly convex by Theorem 6.5. Suppose that $Y, Z \subset X$ are both $G$-orbits such that $\nu \in \mathcal{V}(G / H) \cap \mathcal{C}_{Y}(X)^{\circ} \cap \mathcal{C}_{Z}(X)^{\circ}$ : then $\nu$ has both center $\bar{Y}$ and $\bar{Z}$ on $X$ by Proposition 6.2 , therefore $Y=Z$ because the center is unique. Therefore $\mathfrak{F}(X)$ is a colored fan, which uniquely determines $X$ thanks to Theorem 6.5 .

Suppose that $\mathfrak{F}$ is a strictly convex colored fan. For all colored cones $(\mathcal{C}, \mathcal{F}) \in \mathfrak{F}$, let $G / H \hookrightarrow X_{\mathcal{C}, \mathcal{F}}$ be the simple spherical embedding defined by the colored cone is $(\mathcal{C}, \mathcal{F})$. Let $X$ be the scheme obtained by glueing together all such varieties along their maximal isomorphic open subsets corresponding to the intersection of cones. Notice that $X$ is a normal integral scheme of finite type. Since $X$ contains $G / H$ as an open orbit, to conclude the proof we need to show that $X$ is separated, namely that the diagonal embedding $\iota: X \rightarrow X \times X$ is closed. Let $Y \subset \overline{\iota(X)} \backslash \iota(X)$ be a $G$-orbit, then $Y \subset X_{\mathcal{C}_{1}, \mathcal{F}_{1}} \times X_{\mathcal{C}_{2}, \mathcal{F}_{2}}$ for some colored cones in $\mathfrak{F}(X)$ with $\left(\mathcal{C}_{1}, \mathcal{F}_{1}\right) \neq\left(\mathcal{C}_{2}, \mathcal{F}_{2}\right)$. We may assume that $\left(\mathcal{C}_{1}, \mathcal{F}_{1}\right)$ and $\left(\mathcal{C}_{2}, \mathcal{F}_{2}\right)$ are maximal with this property. Let $X^{\prime}$ be the spherical variety obtained as the normalization of the closure of $G / H$ diagonally embedded in $X_{\mathcal{C}_{1}, \mathcal{F}_{1}} \times X_{\mathcal{C}_{2}, \mathcal{F}_{2}}$ and let $Y^{\prime} \subset X^{\prime}$ be an orbit mapping on $Y$. For $i=1,2$, let $\varphi_{i}: X^{\prime} \rightarrow X_{\mathcal{C}_{i}, \mathcal{F}_{i}}$ be the projection, and set $Y_{i}=\varphi_{i}\left(Y^{\prime}\right)$ : then by the maximality of the correspondng colored cones it follows that $Y_{i} \subset X_{\mathcal{C}_{i}, \mathcal{F}_{i}}$ is the unique closed orbit. Let $\nu \in \mathcal{V}(G / H)$ be a valuation with center $\overline{Y^{\prime}}$ on $X^{\prime}$, then $\nu$ has center $Y_{1}$ on $X_{\mathcal{C}_{1}, \mathcal{F}_{1}}$ and $Y_{2}$ on $X_{\mathcal{C}_{2}, \mathcal{F}_{2}}$. Therefore Proposition 6.2 implies $\nu \in \mathcal{C}_{1}^{\circ} \cap \mathcal{C}_{2}^{\circ}$, hence $\left(\mathcal{C}_{1}, \mathcal{F}_{1}\right)=\left(\mathcal{C}_{2}, \mathcal{F}_{2}\right)$ by $(\mathrm{CF} 2)$, a contradiction.

\section{MORPHISMS BETWEEN SPHERICAL EMBEDDINGS}

Let now $G / H$ and $G / H^{\prime}$ be spherical homogeneous spaces, and assume that $H \subset H^{\prime}$. As usual, we assume that $B H$ is open in $G$. Then we have a surjective equivariant morphism $\varphi: G / H \rightarrow G / H^{\prime}$. The inclusion $\varphi^{*}: \mathrm{k}\left(G / H^{\prime}\right) \hookrightarrow \mathrm{k}(G / H)$ induces an injection $\varphi^{*}: \Lambda\left(G / H^{\prime}\right) \hookrightarrow \Lambda(G / H)$, hence a surjection $\varphi_{*}: \mathcal{Q}(G / H) \rightarrow$ $\mathcal{Q}\left(G / H^{\prime}\right)$. Notice that if we restrict $\varphi_{*}$ to the valuation cone, then by Corollary 4.6 we get a surjective map

$$
\varphi_{*}: \mathcal{V}(G / H) \rightarrow \mathcal{V}\left(G / H^{\prime}\right) .
$$

Lastly, if $\Delta_{\varphi} \subset \Delta(G / H)$ is the set of colors which project dominantly onto $G / H^{\prime}$, then we have a map (that we still denote by the same symbol)

$$
\varphi_{*}: \Delta(G / H) \backslash \Delta_{\varphi} \rightarrow \Delta\left(G / H^{\prime}\right)
$$

Proposition 8.1. Suppose that $H \subset H^{\prime} \subset \mathrm{N}_{G}(H)$, then

$$
\Lambda(G / H) / \Lambda\left(G / H^{\prime}\right) \simeq \mathcal{X}\left(H^{\prime} / H\right) .
$$

Proof Notice that $B H=B H^{\prime}$ : indeed for all $g \in H^{\prime}$ both $B H$ and $B H g^{-1}$ are open in $G$ and homogeneous under $B \times H$, hence they coincide. On the other hand as $B$-varieties we have $B H / H \simeq B / B \cap H$ and $B H^{\prime} / H^{\prime} \simeq B / B \cap H^{\prime}$, therefore $H^{\prime} / H \simeq B \cap H^{\prime} / B \cap H$.

Fix maximal diagonalizable subgroups $S \subset B \cap H$ and $S^{\prime} \subset B \cap H^{\prime}$ such that $S \subset S^{\prime}$, and let $T \subset B$ be a maximal torus such that $S \subset S^{\prime} \subset T$. Remark 2.18 
shows that $\Lambda(G / H) \simeq \mathcal{X}(T / S)$ and $\Lambda\left(G / H^{\prime}\right) \simeq \mathcal{X}\left(T / S^{\prime}\right)$. On the other hand, the exact sequence $1 \rightarrow S^{\prime} / S \rightarrow T / S \rightarrow T / S^{\prime} \rightarrow 1$ induces an exact sequence $1 \rightarrow \mathcal{X}\left(T / S^{\prime}\right) \rightarrow \mathcal{X}(T / S) \rightarrow \mathcal{X}\left(S^{\prime} / S\right) \rightarrow 1$ (see [1, Proposition 8.2]), therefore $\Lambda(G / H) / \Lambda\left(G / H^{\prime}\right) \simeq \mathcal{X}\left(S^{\prime} / S\right)$.

Again, by Remark 2.18 it follows that $\mathcal{X}\left(S^{\prime} / S\right)$ is the set of $\left(B \cap H^{\prime}\right)$-weights occurring in $\mathrm{k}\left(B \cap H^{\prime} / B \cap H\right)^{\left(B \cap H^{\prime}\right)}$. Since $B \cap H$ is normal in $B \cap H^{\prime}$, this set is precisely $\mathcal{X}\left(B \cap H^{\prime} / B \cap H\right) \simeq \mathcal{X}\left(H^{\prime} / H\right)$.

Corollary 8.2. Suppose that $\left[H^{\prime}: H\right]<\infty$, then $\Lambda\left(G / H^{\prime}\right) \subset \Lambda(G / H)$ is a sublattice of finite index and $\mathcal{V}(G / H)=\mathcal{V}\left(G / H^{\prime}\right)$ under the natural identification $\mathcal{Q}(G / H)=\mathcal{Q}\left(G / H^{\prime}\right)$.

Proof Notice that $H$ and $H^{\prime}$ have the same identity component $H^{0}$, which is a spherical subgroup of $G$. Therefore we may assume that $H$ is connected. Then $H$ is a normal subgroup of $H^{\prime}$, and by previous proposition we get $\Lambda(G / H) / \Lambda\left(G / H^{\prime}\right) \simeq$ $\mathcal{X}\left(H^{\prime} / H\right)$.

We now define morphisms between colored cones.

Definition 8.3. $\quad$ i) Let $(\mathcal{C}, \mathcal{F})$ and $\left(\mathcal{C}^{\prime}, \mathcal{F}^{\prime}\right)$ be colored cones resp. for $G / H$ and $G / H^{\prime}$, then we say that $\varphi_{*}$ maps $(\mathcal{C}, \mathcal{F})$ to $\left(\mathcal{C}^{\prime}, \mathcal{F}^{\prime}\right)$ if the following inclusions hold:

$(\mathrm{CM} 1) \varphi_{*}(\mathcal{C}) \subset \mathcal{C}^{\prime}$

(CM2) $\varphi_{*}\left(\mathcal{F} \backslash \Delta_{\varphi}\right) \subset \mathcal{F}^{\prime}$.

ii) Let $\mathfrak{F}$ and $\mathfrak{F}^{\prime}$ be colored fans for $G / H$ and $G / H^{\prime}$ respectively, then we say that $\varphi_{*}$ maps $\mathfrak{F}$ to $\mathfrak{F}^{\prime}$ if every element of $\mathfrak{F}$ maps to some element of $\mathfrak{F}^{\prime}$.

Remark 8.4. Notice that, if $\mathfrak{F}$ and $\mathfrak{F}^{\prime}$ are colored fans for $G / H$, then $\mathfrak{F}$ maps to $\mathfrak{F}^{\prime}$ if and only if every maximal element of $\mathfrak{F}$ maps to some element of $\mathfrak{F}^{\prime}$.

Theorem 8.5 ([17, Theorem 5.1]). Let $\varphi: G / H \rightarrow G / H^{\prime}$ be a surjective equivariant morphism, and let $G / H \hookrightarrow X$ and $G / H^{\prime} \hookrightarrow X^{\prime}$ be spherical embeddings. Then $\varphi$ lifts to a morphism $X \rightarrow X^{\prime}$ if and only if $\varphi_{*}$ maps $\mathfrak{F}(X)$ to $\mathfrak{F}\left(X^{\prime}\right)$.

Proof We may assume that both $X$ and $X^{\prime}$ are simple embeddings, with closed orbits resp. $Y$ and $Y^{\prime}$. Suppose that $\varphi$ extends to a morphism $\varphi: X \rightarrow X^{\prime}$. Then $Y^{\prime} \subset \overline{\varphi(Y)}$, therefore $Y^{\prime} \subset \overline{\varphi(D)}$ for all $D \in \Delta_{Y}(X)$, and we get (CM2). On the other hand $Y^{\prime}$ is contained in every $G$-stable divisor of $X^{\prime}$, thus the same argument as above shows that $\varphi^{-1}\left(X^{\prime} \backslash X_{Y^{\prime}, B}^{\prime}\right) \subset X \backslash X_{Y, B}$. Therefore $\varphi$ restricts to a morphism $X_{Y, B} \rightarrow X_{Y^{\prime}, B}^{\prime}$, hence we get an inclusion $\mathrm{k}\left[X_{Y^{\prime}, B}^{\prime}\right]^{(B)} \hookrightarrow \mathrm{k}\left[X_{Y, B}\right]^{(B)}$ and (CM1) follows by Proposition 6.2.

Suppose now that $\left(\mathcal{C}_{Y}(X), \Delta_{Y}(X)\right)$ maps to $\left(\mathcal{C}_{Y^{\prime}}\left(X^{\prime}\right), \Delta_{Y^{\prime}}\left(X^{\prime}\right)\right)$ and define $U_{0}=$ $G / H \cap X_{Y, B}$ and $U_{0}^{\prime}=G / H^{\prime} \cap X_{Y^{\prime}, B}^{\prime}$. Since $\varphi(D)=B H^{\prime} / H^{\prime}$ for all $D \in \Delta_{\varphi}$, by (CM2) the restriction of $\varphi$ gives a dominant morphism $U_{0} \rightarrow U_{0}^{\prime}$, hence an inclusion $\mathrm{k}\left[U_{0}^{\prime}\right] \hookrightarrow \mathrm{k}\left[U_{0}\right]$. On the other hand since $X$ and $X^{\prime}$ are normal, a function $f \in \mathrm{k}\left[U_{0}\right]$ (resp. $f \in \mathrm{k}\left[U_{0}^{\prime}\right]$ ) extends to $\mathrm{k}\left[X_{Y, B}\right]$ (resp. to $\mathrm{k}\left[X_{Y^{\prime}, B}^{\prime}\right]$ ) if and only if $\nu(f) \geqslant 0$ for all $\nu \in \mathcal{V}(G / H) \cap \mathcal{C}_{Y}(X)$ (resp. for all $\nu \in \mathcal{V}\left(G / H^{\prime}\right) \cap \mathcal{C}_{Y^{\prime}}\left(X^{\prime}\right)$ ). Since $\varphi_{*}(\mathcal{V}(G / H))=\mathcal{V}\left(G / H^{\prime}\right)$, it follows by (CM1) that every $f \in \mathrm{k}\left[U_{0}^{\prime}\right]$ which extends to $X_{Y^{\prime}, B}^{\prime}$ extends to $X_{Y, B}$ as well. Therefore $\varphi$ induces an inclusion $\mathrm{k}\left[X_{Y^{\prime}, B}^{\prime}\right] \hookrightarrow$ $\mathrm{k}\left[X_{Y, B}\right]$, hence a morphism $X_{Y, B} \rightarrow X_{Y^{\prime}, B}^{\prime}$. Since $X=G X_{Y, B}$ and $X^{\prime}=G X_{Y^{\prime}, B}^{\prime}$, it follows that $\varphi$ extends to a morphism $X \rightarrow X^{\prime}$.

8.1. Proper morphisms. We now turn to the characterization of proper morphisms of spherical varieties.

Definition 8.6. Let $\mathfrak{F}$ be a colored fan. The support of $\mathfrak{F}$ is

$$
\operatorname{supp} \mathfrak{F}=\bigcup_{(\mathcal{C}, \mathcal{F}) \in \mathfrak{F}} \mathcal{C} \cap \mathcal{V}(G / H)
$$


Notice that, if $X$ is a spherical variety, then by Proposition $6.2 \operatorname{supp} \mathfrak{F}(X)$ is the set of the invariant valuations on $X$ which have a center. Notice also that if $X \rightarrow X^{\prime}$ is an equivariant morphism between two spherical embeddings $G / H \hookrightarrow X$ and $G / H^{\prime} \hookrightarrow X^{\prime}$, then by Theorem 8.5 we have $\varphi_{*}(\operatorname{supp} \mathfrak{F}(X)) \subset \operatorname{supp} \mathfrak{F}\left(X^{\prime}\right)$.

Theorem 8.7 ([17, Theorem 5.2]). Let $G / H \hookrightarrow X$ and $G / H^{\prime} \hookrightarrow X^{\prime}$ be spherical embeddings and suppose that $\varphi: X \rightarrow X^{\prime}$ is an equivariant dominant morphism. Then $\varphi$ is proper if and only if

$$
\operatorname{supp} \mathfrak{F}(X)=\mathcal{V}(G / H) \cap \varphi_{*}^{-1}\left(\operatorname{supp} \mathfrak{F}\left(X^{\prime}\right)\right) .
$$

In particular, $X$ is complete if and only if $\operatorname{supp} \mathfrak{F}(X)=\mathcal{V}(G / H)$.

Proof Suppose that $\varphi$ is proper, then by the valuative criterion of properness for every valuation $\operatorname{ring} R$ with fraction field $K$ and for every commutative diagram

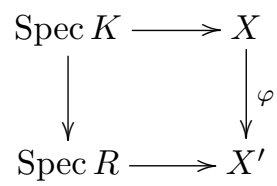

there exists a unique morphism $\operatorname{Spec} R \rightarrow X$ making the diagram commute. Let $\nu^{\prime} \in \operatorname{supp} \mathfrak{F}\left(X^{\prime}\right)$, let $Y^{\prime} \subset X^{\prime}$ be the open $G$-orbit of the center of $\nu^{\prime}$ and let $\nu \in \mathcal{V}(G / H)$ be a valuation extending $\nu^{\prime}$. By Proposition 4.3 we have $\mathrm{k}\left[X_{Y^{\prime}, B}^{\prime}\right] \subset$ $\mathcal{O}_{\nu^{\prime}} \subset \mathcal{O}_{\nu}$, hence we get a morphism $\operatorname{Spec} \mathcal{O}_{\nu} \rightarrow X_{Y^{\prime}, B}$. Since $\varphi$ is proper, we get a morphism $\operatorname{Spec} \mathcal{O}_{\nu} \rightarrow X$. Therefore $\nu$ has a center on $X$ as well, hence $\nu \in \operatorname{supp} \mathfrak{F}(X)$.

Suppose conversely that $\operatorname{supp} \mathfrak{F}(X)=\mathcal{V}(G / H) \cap \varphi_{*}^{-1}\left(\operatorname{supp} \mathfrak{F}\left(X^{\prime}\right)\right)$, by the following lemma there exists an equivariant open embedding $X \subset X_{1}$ together with a proper morphism $\varphi_{1}: X_{1} \rightarrow X^{\prime}$ extending $\varphi$. Because $\varphi_{1}$ is proper we have $\operatorname{supp} \mathfrak{F}\left(X_{1}\right)=\mathcal{V}(G / H) \cap\left(\varphi_{1}\right)_{*}^{-1}\left(\operatorname{supp} \mathfrak{F}\left(X^{\prime}\right)\right)$, therefore by the assumption we get $\operatorname{supp} \mathfrak{F}\left(X_{1}\right)=\operatorname{supp} \mathfrak{F}(X)$. In particular, for every $G$-orbit $Y_{1} \subset X_{1}$ we must have $\mathcal{V}(G / H) \cap \mathcal{C}_{Y_{1}}\left(X_{1}\right)^{\circ} \cap \mathcal{C}_{Y}(X) \neq \varnothing$ for some $G$-orbit $Y \subset X$, hence $\mathcal{C}_{Y_{1}}\left(X_{1}\right)$ is a face of $\left(\mathcal{C}_{Y}\left(X_{1}\right), \Delta_{Y}\left(X_{1}\right)\right)$ and so $Y \subset \overline{Y_{1}}$. Since $X_{1} \backslash X$ is closed, it follows that it must be empty, hence $X=X_{1}$ and $\varphi$ is proper.

Lemma 8.8. Let $\varphi: X \rightarrow X^{\prime}$ be an equivariant morphism of $G$-varieties, then there exists a open embedding $X \hookrightarrow X_{1}$ into a normal $G$-variety $X_{1}$ such that $\varphi$ extends to a proper equivariant morphism $\varphi_{1}: X_{1} \rightarrow X^{\prime}$.

Proof Let $X \hookrightarrow \bar{X}$ and $X^{\prime} \hookrightarrow \overline{X^{\prime}}$ be two equivariant completions (which exist after a theorem of Sumihiro, see [32, Theorem 3]), and let $X_{2}$ be the normalization of the closure of the image of $X$ mapped diagonally in $\bar{X} \times \overline{X^{\prime}}$. Then $X_{2}$ is a complete $G$-variety containing $X$ as an open subset, and the second projection gives a proper morphism $\varphi_{2}: X_{2} \rightarrow \overline{X^{\prime}}$ which extends $\varphi$. Therefore we get the desired embedding of $X$ by setting $X_{1}=\varphi_{2}^{-1}\left(X^{\prime}\right)$ and $\varphi_{1}=\varphi_{2 \mid X_{1}}$.

We now introduce an important class of spherical embeddings. Their name is due to the fact that, if $\operatorname{char}(k)=0$, their local structure reduces to that of a toric variety (see [26, Theorem 3.4.1]) (in which case they are also toroidal in the sense of $[16])$.

Definition 8.9. A spherical embedding $G / H \hookrightarrow X$ is called toroidal if $\Delta_{Y}(X)=\varnothing$ for every $G$-orbit $Y \subset X$.

Suppose that $G / H \hookrightarrow X$ is a toroidal embedding of a spherical homogeneous space $G / H$ and let $Y \subset X$ be a closed orbit, then by definition we have $\mathcal{C}_{Y}(X) \subset$ $\mathcal{V}(G / H)$. If moreover $X$ is complete and toroidal, then by Theorem 8.7 it follows 
that $\mathcal{V}(G / H)=\bigcup_{Y} \mathcal{C}_{Y}(X)$, where $Y$ runs among the closed orbits of $X$. In particular, every complete toroidal embeddings corresponds to a subdivision of $\mathcal{V}(G / H)$ into strictly convex cones, and a simple complete toroidal embedding exists if and only if $\mathcal{V}(G / H)$ is a strictly convex cone. In general such an embedding need not exist, on the other hand complete toroidal embeddings always do exist.

Proposition 8.10 ([7, §3.1], [17, Lemma 6.2]). Let $G / H$ be a spherical homogeneous space, then $G / H$ admits a complete toroidal embedding.

Proof Let $\pi: G \rightarrow G / H$ be the projection. Let $f_{0} \in \mathrm{k}[G]^{(B \times H)}$ be a function vanishing on $\pi^{-1}(D)$ for all $D \in \Delta(G / H)$ and let $V \subset \mathrm{k}[G]$ be the $G$-module generated by $f_{0}$. Since the left action of $G$ commutes with the right action of $H$, the evaluation of functions induces an equivariant morphism $G / H \rightarrow \mathbb{P}\left(V^{*}\right)$. Let $X^{\prime}$ be the closure of the image of $G / H$ and let $\psi: G / H \rightarrow X^{\prime}$ be the corresponding morphism. Let $Z \subset X^{\prime}$ be the zero locus of $f_{0 \mid X^{\prime}}$. Notice that by construction $\psi(D) \subset Z$ for all $D \in \Delta(G / H)$, and that $Z$ contains no $G$-orbit: if indeed $Y \subset Z$ is a $G$-orbit, then $\left(g . f_{0}\right)_{\left.\right|_{Y}}=0$ for all $g \in G$, hence $f_{\left.\right|_{Y}}=0$ for all $f \in V$.

Let now $G / H \hookrightarrow X^{\prime \prime}$ be an arbitrary completion, and consider the diagonal morphism $\psi^{\prime}: G / H \hookrightarrow X^{\prime} \times X^{\prime \prime}$. Then we get a complete and toroidal embedding $G / H \hookrightarrow X$ by taking $X$ the normalization of $\overline{\psi^{\prime}(G / H)}$.

We will study more closely the structure of $\mathcal{V}(G / H)$ in Sections 10 and 11 . There we will se that $\mathcal{V}(G / H)$ is indeed a convex cone (Theorem 10.5), which is strictly convex if and only if $H$ has finite index in its normalizer (Theorem 11.1).

Example 8.11 $\left(\mathrm{SL}_{2} / T\right.$, part II). We continue with Example 3.3. Consider the natural left action of $\mathrm{SL}_{2}$ on $\mathbb{P}^{1}$, and consider the corresponding diagonal action on $\mathbb{P}^{1} \times \mathbb{P}^{1}$. Let $\left(\left[\begin{array}{l}a_{11} \\ a_{21}\end{array}\right],\left[\begin{array}{l}a_{12} \\ a_{22}\end{array}\right]\right)$ be homogeneous coordinates on $\mathbb{P}^{1} \times \mathbb{P}^{1}$ and set $x_{0}=\left(\left[\begin{array}{l}1 \\ 0\end{array}\right],\left[\begin{array}{l}0 \\ 1\end{array}\right]\right)$. Then the isotropy group of $x_{0}$ is $T$, and the $\mathrm{SL}_{2}$-orbit of $x_{0}$ is

$$
\mathcal{O}=\left\{(p, q) \in \mathbb{P}^{1} \times \mathbb{P}^{1} \mid p \neq q\right\} \simeq \mathrm{SL}_{2} / T \text {. }
$$

Notice that $\left(\mathbb{P}^{1} \times \mathbb{P}^{1}\right) \backslash \mathcal{O}=\operatorname{diag}\left(\mathbb{P}^{1}\right)$ is a single $\mathrm{SL}_{2}$-orbit of codimension one. Therefore $\mathbb{P}^{1} \times \mathbb{P}^{1}$ is a spherical embedding of $\mathrm{SL}(2) / T$ which is complete, simple and toroidal. In particular it follows that $\mathcal{V}\left(\mathrm{SL}_{2} / T\right) \subset \mathcal{Q}\left(\mathrm{SL}_{2} / N\right)$ is a strictly convex cone. Consider the rational function $f^{\prime}=\frac{a_{11} a_{22}-a_{12} a_{22}}{a_{21} a_{22}}$ on $\mathbb{P}^{1} \times \mathbb{P}^{1}$ : then $f_{\mid \mathcal{O}}^{\prime}=\frac{1}{f}$, where $f \in \mathrm{k}\left[\mathrm{SL}_{2} / T\right]^{(B)}$ is the function of weight $\alpha$ defined in Example 3.3. If $\nu_{0} \in \mathcal{V}\left(\mathrm{SL}_{2} / T\right)$ is the $\mathrm{SL}_{2}$-invariant valuation associated to $\operatorname{diag}\left(\mathbb{P}^{1}\right)$, it follows then $\left\langle\nu_{0}, \alpha\right\rangle=\nu_{0}(f)=\nu_{0}\left(\frac{1}{f^{\prime}}\right)=-1$. Since it is strictly convex and $\operatorname{dim} \mathcal{Q}\left(\mathrm{SL}_{2} / T\right)=1$, the valuation cone $\mathcal{V}\left(\mathrm{SL}_{2} / T\right)$ coincides with the cone generated by $\nu_{0}$, therefore

$$
\mathcal{V}\left(\mathrm{SL}_{2} / T\right)=\left\{\nu \in \mathcal{Q}\left(\mathrm{SL}_{2} / N\right) \mid\langle\nu, \alpha\rangle \leqslant 0\right\} .
$$

As an immediate application of Theorem 7.4, notice that, up to equivariant isomorphism, $\mathbb{P}^{1} \times \mathbb{P}^{1}$ is the unique nontrivial equivariant embedding of $\mathrm{SL}_{2} / T$. Indeed, since $\rho\left(D^{+}\right)=\rho\left(D^{-}\right)=\frac{1}{2} \alpha^{\vee}$, by the description of $\mathcal{V}\left(\mathrm{SL}_{2} / T\right)$ it follows that $\left(\mathcal{V}\left(\mathrm{SL}_{2} / T\right), \varnothing\right)$ is the unique nontrivial colored cone for $\mathrm{SL}_{2} / T$.

Example 8.12 $\left(\mathrm{SL}_{2} / N(T)\right)$. Keep the notation of the previous example. Let $N=T \cup \dot{s}_{\alpha} T$ be the normalizer of $T \subset \mathrm{SL}_{2}$ and consider the homogeneous space $\mathrm{SL}_{2} / N$ : since $\mathrm{SL}_{2} / T$ is spherical, it follows that $\mathrm{SL}_{2} / N$ is spherical as well. By Proposition 8.1 it follows that $\Lambda\left(\mathrm{SL}_{2} / N\right) \subset \Lambda\left(\mathrm{SL}_{2} / T\right)$ is a sublattice of index two, hence

$$
\Lambda\left(\mathrm{SL}_{2} / N\right)=2 \Lambda\left(\mathrm{SL}_{2} / T\right)=2 \mathbb{Z} \alpha
$$

By the discussion preceeding Proposition 8.1 the subset of invariant valuations $\mathcal{V}\left(\mathrm{SL}_{2} / N\right)$ coincides with that of $\mathrm{SL}_{2} / T$, namely

$$
\mathcal{V}\left(\mathrm{SL}_{2} / N\right)=\left\{\nu \in \mathcal{Q}\left(\mathrm{SL}_{2} / N\right) \mid\langle\nu, \alpha\rangle \leqslant 0\right\} .
$$


Notice that $\mathrm{SL}_{2} / N$ decomposes in two $B$-orbits: indeed the two colors $D^{+}$and $D^{-}$ of $\mathrm{SL}_{2} / T$ have the same image in $\mathrm{SL}_{2} / N$, which therefore possesses a unique color $D=B N / N$. Let $f \in \mathrm{k}\left[\mathrm{SL}_{2} / T\right]^{(B)}$ be the function of weight $\alpha$ defined in Example 3.3. Then $f^{2} \in \mathrm{k}\left[\mathrm{SL}_{2} / N\right]^{(B)}$ and $\langle\rho(D), 2 \alpha\rangle=\nu_{D}\left(f^{2}\right)=2$, namely $\rho(D)=\frac{1}{2} \alpha^{\vee}$. As in the previous example, we see that (up to equivariant isomorphisms) $\mathrm{SL}_{2} / N$ admits a unique nontrivial equivariant embedding, corresponding to the colored cone $\left(\mathcal{V}\left(\mathrm{SL}_{2} / N\right), \varnothing\right)$ : indeed this is the unique nontrivial colored cone for $\mathrm{SL}_{2} / N$. To construct it geometrically, consider the representation of $\mathrm{SL}_{2}$ on the vector space $V=\mathrm{k}[x, y]_{2}$ considered in Example 3.3, consider the induced action on $\mathbb{P}^{2}=\mathbb{P}(V)$ and set $x_{0}=[x y]$. Then the stabilizer of $x_{0}$ coincides with $N$ and the orbit of $x_{0}$ is

$$
\mathcal{O}=\{[f] \in \mathbb{P}(V) \mid \delta(f) \neq 0\}
$$

where $\delta(f)$ denotes the discriminant of $f$. Since $\mathbb{P}^{2} \backslash \mathcal{O} \simeq \mathbb{P}^{1}$, it follows that $\mathbb{P}^{2}$ is a complete, simple, and toroidal embedding of $\mathrm{SL}_{2} / N$.

\section{Co-CONNECTED INCLUSIONS AND COLORED SUBSPACES}

Suppose that $H \subset H^{\prime} \subset G$ are spherical subgroups, we say that $H$ is co-connected in $H^{\prime}$ if $H^{\prime} / H$ is connected. In this section we will classifiy co-connected inclusions by means of colored data. Fix a co-connected inclusion $H \subset H^{\prime}$ and let $\varphi: G / H \rightarrow$ $G / H^{\prime}$ be the projection. Denote the corresponding base points by $x_{0}=H / H$ and $x_{0}^{\prime}=H^{\prime} / H^{\prime}$ (which are by assumption in the respective open $B$-orbits) and define

$$
\mathcal{C}_{\varphi}=\left\{\nu \in \mathcal{Q}(G / H) \mid\langle\nu, \lambda\rangle=0 \text { for all } \lambda \in \Lambda\left(G / H^{\prime}\right)\right\} .
$$

Definition 9.1. A colored subspace for $G / H$ is a colored cone $(\mathcal{C}, \mathcal{F})$ such that $\mathcal{C}$ is a subspace of $\mathcal{Q}(G / H)$.

Notice that if $\mathcal{C} \subset \mathcal{Q}(G / H)$ is a subspace and $\mathcal{F} \subset \Delta(G / H)$, then $(\mathcal{C}, \mathcal{F})$ is a colored subspace if and only if $\mathcal{C}$ is generated as a convex cone by $\rho(\mathcal{F})$ and by finitely many $\nu \in \mathcal{V}(G / H)$.

Proposition 9.2 ([17, Lemma 5.3]). Let $H \subset H^{\prime} \subset G$ be a co-connected inclusion of spherical subgroups and let $\varphi: G / H \rightarrow G / H^{\prime}$ be the projection.

i) Denote $U_{0}=G / H \backslash \bigcup_{D \in \Delta(G / H) \backslash \Delta_{\varphi}} D$, then

$$
\mathrm{k}\left[B x_{0}^{\prime}\right]=\left\{f \in \mathrm{k}\left[U_{0}\right] \mid \nu(f) \geqslant 0 \text { for all } \nu \in \mathcal{V}(G / H) \cap \mathcal{C}_{\varphi}\right\}
$$

ii) The pair $\left(\mathcal{C}_{\varphi}, \Delta_{\varphi}\right)$ is a colored subspace. Moreover, the colored data of $G / H$ is recovered from that of $G / H^{\prime}$ as follows: $\Lambda\left(G / H^{\prime}\right)=\Lambda(G / H) \cap \mathcal{C}_{\varphi}^{\perp}$, $\mathcal{Q}\left(G / H^{\prime}\right)=\mathcal{Q}(G / H) / \mathcal{C}_{\varphi}, \Delta\left(G / H^{\prime}\right)=\Delta(G / H) \backslash \Delta_{\varphi}$.

Proof By Lemma 8.8, there exists a spherical embedding $G / H \hookrightarrow X$ such that $\varphi$ extends to a proper morphism $\bar{\varphi}: X \rightarrow G / H^{\prime}$. Let $X_{0}=\bar{\varphi}^{-1}\left(B x_{0}^{\prime}\right)$. Since $H^{\prime} / H$ is connected, by the Stein factorization (see [12, Theorem 12.69]) it follows that $\bar{\varphi}_{*} \mathcal{O}_{X}=\mathcal{O}_{G / H^{\prime}}$, hence $\mathrm{k}\left[B x_{0}^{\prime}\right]=\mathrm{k}\left[X_{0}\right]$.

Notice that $X_{0}$ intersects every $G$-orbit of $X$, therefore $\overline{X_{0} \backslash G x_{0}}=X \backslash G x_{0}$. Since $X$ is normal, it follows that a function $f \in \mathrm{k}\left[U_{0}\right]$ extends to $X_{0}$ if and only if $\nu(f) \geqslant 0$ for all $\nu \in \mathcal{V}(G / H)$ with center on $X$, namely for all $\nu \in \operatorname{supp} \mathfrak{F}(X)$. Since $\bar{\varphi}$ is proper, by Theorem 8.7 we have

$$
\operatorname{supp} \mathfrak{F}(X)=\mathcal{V}(G / H) \cap \varphi_{*}^{-1}(0)=\mathcal{V}(G / H) \cap \mathcal{C}_{\varphi},
$$

hence $\mathrm{k}\left[X_{0}\right]=\left\{f \in \mathrm{k}\left[U_{0}\right] \mid \nu(f) \geqslant 0\right.$ for all $\left.\nu \in \mathcal{V}(G / H) \cap \mathcal{C}_{\varphi}\right\}$.

Notice that in the previous description we onky need finitely many inequalities namely those coming from a $G$-stable divisor of $X$. On the other hand, since $U_{0}$ is normal we have

$$
\mathrm{k}\left[U_{0}\right]=\left\{f \in \mathrm{k}\left[B x_{0}\right] \mid \nu_{D}(f) \geqslant 0 \text { for all } D \in \Delta_{\varphi}\right\},
$$


and since $\mathrm{k}\left[B x_{0}^{\prime}\right]=\mathrm{k}\left[X_{0}\right]$ we get $\Lambda\left(G / H^{\prime}\right)=\Lambda(G / H) \cap \mathcal{C}^{\vee}$, where $\mathcal{C} \subset \mathcal{Q}(G / H)$ is the cone generated by $\rho\left(\Delta_{\varphi}\right)$ together with the valuations defined by the $G$ stable divisors of $X$. Notice that $\rho\left(\Delta_{\varphi}\right) \subset \mathcal{C}_{\varphi}$, therefore $\mathcal{C} \subset \mathcal{C}_{\varphi}$. On the other hand we have by definition $\Lambda\left(G / H^{\prime}\right) \subset \mathcal{C}_{\varphi}^{\perp}$, therefore $\mathcal{C}^{\vee} \subset \mathcal{C}_{\varphi}^{\vee}$ and it follows $\mathcal{C}_{\varphi}=\mathcal{C}$. Therefore $\left(\mathcal{C}_{\varphi}, \Delta_{\varphi}\right)$ is a colored subspace, and the other claims follow straightforwardly.

Theorem 9.3 ([17, Theorem 5.4]). Let $G / H$ be a spherical homogeneous space. Then the map which associates to $H^{\prime}$ the colored subspace $\left(\mathcal{C}_{\varphi}, \Delta_{\varphi}\right)$ defined by $\varphi$ : $G / H \rightarrow G / H^{\prime}$ is an order preseving bijection

$$
\left\{\text { Co-connected inclusions } H^{\prime} \supset H\right\} \longleftrightarrow\{\text { Colored subspaces for } G / H\} \text {. }
$$

Proof Arguing as in Theorem 6.1, by making use of Proposition 9.2 instead of Proposition 6.2 one shows that, for all co-connected inclusion $H \subset H^{\prime}$, the projection $\varphi: G / H \rightarrow G / H^{\prime}$ is uniquely determined up to equivariant isomorphism by the colored subspace $\left(\mathcal{C}_{\varphi}, \Delta_{\varphi}\right)$. More precisely, if $H \subset H^{\prime \prime}$ is another co-connected inclusion defining the same colored subspace, then one shows that $H^{\prime \prime}=g H^{\prime} g^{-1}$ for some $g \in \mathrm{N}_{G}(H)$ with $g \cdot \Delta_{\varphi}=\Delta_{\varphi}$. On the other hand, if $g \in \mathrm{N}_{G}(H)$ is such an element and $\psi_{g}$ is the corresponding equivariant automorphism of $G / H$, by the first part of Proposition 9.2 we see that $\psi_{g}$ induces an equivariant automorphism $\psi_{g}^{*}: \mathrm{k}\left[B x_{0}^{\prime}\right] \rightarrow \mathrm{k}\left[B x_{0}^{\prime}\right]$, hence an equivariant automorphism of the open $B$-orbit of $G / H^{\prime}$. Since the latter extends to an equivariant automorphism of $G / H^{\prime}$, it follows that $g \in \mathrm{N}_{G}\left(H^{\prime}\right)$, hence $H^{\prime \prime}=H^{\prime}$.

Conversely, arguing as in the proof of Theorem 6.5 one shows that for every colored subspace $(\mathcal{C}, \mathcal{F})$ there exists a co-connected inclusion $H \subset H^{\prime}$ whose colored subspace is precisely $(\mathcal{C}, \mathcal{F})$. Let indeed $D_{0}$ be the union of the colors $D \in \Delta(G / H) \backslash \mathcal{F}$, and let $\pi: G \rightarrow G / H$ be the projection. Since $(\mathcal{C}, \mathcal{F})$ is a colored cone and $\mathcal{C}$ is a vector space, the same construction produces a function $f_{0} \in \mathrm{k}[G]^{(B \times H)}$ vanishing precisely on $\pi^{-1}\left(D_{0}\right)$, and a finite dimensional $G$-module $V \subset \mathrm{k}[G]$ containing $f_{0}$ with an equivariant morphism $\varphi: G / H \rightarrow \mathbb{P}\left(V^{*}\right)$, such that, setting $U_{0}=\left\{x \in \varphi(G / H) \mid f_{0}(x) \neq 0\right\}$, we have

$$
\mathrm{k}\left[U_{0}\right]^{(B)}=\left\{f \in \mathrm{k}(G / H)^{(B)} \mid \lambda_{f} \in \mathcal{C}^{\perp}\right\} .
$$

Let $x_{0} \in G / H$ be the base point corresponding to $H$ and set $x_{0}^{\prime \prime}=\varphi\left(x_{0}\right)$. Then $\mathcal{O}=G x_{0}^{\prime \prime}$ is a homogeneous spherical $G$-variety, and $U_{0}$ is a $B$-stable affine open subset therein. Notice that a color $D \in \Delta(G / H)$ projects dominantly to $\mathcal{O}$ if and only if $D \in \mathcal{F}$ : if indeed $D \in \mathcal{F}$, then $\nu_{D}(f)=0$ for all $f \in \mathrm{k}(G / H)^{(B)}$, therefore $\varphi(D)$ contains the open $B$-orbit of $\mathcal{O}$. On the other hand every color $D \in \Delta(G / H) \backslash \mathcal{F}$ maps in the zero locus of $f_{0}$, therefore $U_{0}=B x_{0}^{\prime \prime}$ is the open $B$-orbit of $\mathcal{O}$.

Let $H^{\prime \prime}$ be the stabilizer of $x_{0}^{\prime \prime}$, and set $H^{\prime}=H\left(H^{\prime \prime}\right)^{0}$. Then $G / H \rightarrow \mathcal{O}$ factors through $G / H^{\prime}$ as follows

$$
G / H \stackrel{\varphi^{\prime}}{\longrightarrow} G / H^{\prime} \stackrel{\psi}{\longrightarrow} \mathcal{O},
$$

where $\varphi^{\prime}$ has connected fibers and $\psi$ has finite fibers. Moreover $\Delta_{\varphi^{\prime}}=\mathcal{F}$, and if $x_{0}^{\prime}=\varphi^{\prime}\left(x_{0}\right)$, then we get $\mathrm{k}\left[B x_{0}^{\prime}\right]^{(B)}=\mathrm{k}\left[B x_{0}^{\prime \prime}\right]^{(B)}$ : indeed we have inclusions $\Lambda(\mathcal{O}) \subset \Lambda\left(G / H^{\prime}\right) \subset \Lambda(G / H)$, and since $\Lambda(\mathcal{O}) \subset \Lambda\left(G / H^{\prime}\right)$ is a sublattice of finite index the equality $\Lambda(\mathcal{O})=\Lambda(G / H) \cap \mathcal{C}^{\perp}$ implies $\Lambda(\mathcal{O})=\Lambda\left(G / H^{\prime}\right)$. Therefore the co-connected inclusion $H \subset H^{\prime}$ defines the prescribed colored subspace.

Combining Theorem 7.4 and Theorem 9.3, we can give a geometrical meaning to all colored fans, even not strictly convex ones. Indeed, let $G / H$ be a spherical homogeneous space and let $\varphi: G / H \rightarrow X$ be a $G$-equivariant dominant morphism to a $G$-variety $X$. Let $x_{0} \in X$ be the corresponding base point and let $H^{\prime} \supset H$ be its stabilizer, so that $\varphi$ factors through $G / H^{\prime}$. Then the morphism $G / H \rightarrow G / H^{\prime}$ 
is surjective and separable, the morphism $G / H^{\prime} \rightarrow X$ is injective and purely inseparable, and the schematic fiber $\varphi^{-1}\left(x_{0}\right)$ is a subscheme of $G / H$ whose underlying reduced subscheme is $H^{\prime} / H$. Therefore the schematic fibers of $\varphi$ are reduced if and only if $\varphi$ is separable, and they are irreducible if and only if $H^{\prime} / H$ is connected. This leads to the following definition.

Definition 9.4. An integral submersion of $G / H$ is a normal variety $X$ with a $G$ equivariant dominant morphism $\varphi: G / H \rightarrow X$ of which all fibers are integral (that is, they are reduced and irreducible).

Suppose that $\varphi: G / H \rightarrow X$ is an integral submersion, if $Y \subset X$ is a $G$-orbit let $\left(\mathcal{C}_{Y}^{\prime}(X), \Delta_{Y}^{\prime}(X)\right)$ be the strictly convex colored cone associated to $Y$ with respect to the spherical embedding $\varphi(G / H) \hookrightarrow X$. Then we can attach to $\varphi$ a colored fan (which is strictly convex if and only if $\varphi$ is birational) by setting

$$
\mathfrak{F}(X)=\left\{\left(\mathcal{C}_{Y}(X), \Delta_{Y}(X)\right)\right\}_{Y \subset X},
$$

where, for every $G$-orbit $Y \subset X$, we denote $\mathcal{C}_{Y}(X)=\varphi_{*}^{-1}\left(\mathcal{C}_{Y}^{\prime}(X)\right)$ and $\Delta_{Y}(X)=$ $\varphi_{*}^{-1}\left(\Delta_{Y}^{\prime}(X)\right)$. Therefore we can summarize Theorem 7.4, Theorem 8.5 and Theorem 9.3 all together in the following theorem.

Theorem 9.5 ([17, Theorem 5.5]). The functor $X \mapsto \mathfrak{F}(X)$ is an equivalence between the category of integral submersions of $G / H$ and the category of colored fans for $G / H$.

\section{The VAluation CONE OF A SPHERICAL HOMOgeneOUS SPACE}

Let $G / H$ be a spherical homogeneous space, we will prove in this section that the set of invariant valuations $\mathcal{V}(G / H) \subset \mathcal{Q}(G / H)$ is a convex cone of maximal dimension. The set of equations defining $\mathcal{V}$ lead us to the definition of the root monoid of $G / H$, which is a submonoid $\Gamma^{+}(G / H) \subset \Lambda(G / H)$ which plays the role of the root monoid of the weight lattice of a root system.

To simplify the notation, we will denote $\Lambda(G / H), \mathcal{V}(G / H), \mathcal{Q}(G / H)$ simply by $\Lambda, \mathcal{V}$ and $\mathcal{Q}$.

Let $f_{1}, \ldots, f_{s} \in \mathrm{k}[G]^{(H)}$, and let $M_{i} \subset \mathrm{k}[G]^{(H)}$ be the $G$-submodule generated by $f_{i}$. We denote by $M_{1} \cdots M_{s} \subset \mathrm{k}[G]$ the $G$-module generated by all possibile products $g_{1} \cdots g_{s}$ with $g_{i} \in M_{i}$ for $i=1, \ldots, s$.

Suppose that $f \in M_{1} \cdots M_{s}$. Then $f \in \mathrm{k}[G]^{(H)}$ has the same $H$-weight of the product $f_{1} \cdots f_{s}$, hence $f_{1} \cdots f_{s} f^{-1} \in \mathrm{k}(G)^{H}=\mathrm{k}(G / H)$. Since $f$ is a product of linear combinations of the shape $\sum_{j} g_{j} f_{i}$ with $g_{j} \in G$, if $\bar{\nu} \in \mathcal{V}(G)$ it follows that $\bar{\nu}(f) \geqslant \bar{\nu}\left(f_{1}\right)+\ldots+\bar{\nu}\left(f_{s}\right)$. Since every $\nu \in \mathcal{V}$ can be extended to $\mathcal{V}(G)$, keeping the previous notation it follows that

$$
\nu\left(f_{1} \cdots f_{s} f^{-1}\right) \leqslant 0 \quad \forall \nu \in \mathcal{V}, \quad \forall f_{1}, \ldots, f_{s} \in \mathrm{k}[G]^{(H)}, \quad \forall f \in M_{1} \cdots M_{s}
$$

If moreover $f_{i} \in \mathrm{k}[G]^{(B \times H)}$ for all $i=1, \ldots, s$ and $f \in\left(M_{1} \cdots M_{s}\right)^{(B)}$, then $f_{1} \cdots f_{s} f^{-1} \in \mathrm{k}(G / H)^{(B)}$. If $\gamma=\gamma\left(f_{1}, \ldots, f_{s}, f\right)$ is the corresponding weight defined by

$$
\gamma=\lambda_{f_{1}}+\ldots+\lambda_{f_{s}}-\lambda_{f}
$$

we get that every $\nu \in \mathcal{V} \subset \mathcal{Q}$ satisfies the inequality $\langle\nu, \gamma\rangle \leqslant 0$.

Definition 10.1. Let $\Gamma_{0}^{+} \subset \Lambda$ be the set of weights $\gamma=\gamma\left(f_{1}, \ldots, f_{s}, f\right)$, where $f_{1}, \ldots, f_{s}, f$ run over all possible choices in $\mathrm{k}[G]^{(B \times H)}$ as above. The root lattice of $G / H$ (denoted by $\Gamma(G / H)$ or simply by $\Gamma$ ), is the sublattice $\Gamma \subset \Lambda$ generated by $\Gamma_{0}^{+}$. The root monoid of $G / H$ (denoted by $\Gamma^{+}(G / H)$ or simply by $\Gamma^{+}$) is the monoid $\Gamma \cap \operatorname{cone}\left(\Gamma_{0}^{+}\right)$. 
Remark 10.2. Regard $\Lambda$ as a sublattice of $\mathcal{X}(T)$. Then $\Gamma^{+}$is contained in the monoid generated by the positive roots: let indeed $\gamma \in \Gamma^{+}$and suppose that $\gamma=$ $\gamma\left(f_{1}, \ldots, f_{s}, f\right)$. Since $f_{1}, \ldots, f_{s} \in \mathrm{k}[G]^{(B)}$, it follows that $f_{i}$ is a highest weight vector in the corresponding $G$-module $M_{i}$ : in particular, if $\lambda_{i}$ is the $B$-weight of $f_{i}$ and if $\mu$ is any $T$-weight in $M_{i}$, the difference $\lambda_{i}-\mu$ is a sum of positive roots. Therefore for every $T$-weight $\mu$ occurring in $M_{1}^{n_{1}} \cdots M_{s}^{n_{s}}$ the difference $\sum n_{i} \lambda_{i}-\mu$ is a sum of positive roots.

Example 10.3. Suppose that $H=B$, then $\Lambda=0$ therefore $\Gamma^{+}=0$ as well.

Example 10.4 (Semisimple groups). We continue with Example 3.6, in particular we will assume that $\operatorname{char}(\mathrm{k})=0$. Suppose that $G$ is a semisimple group, and recall that the weight lattice $\Lambda$ of $(G \times G) / \operatorname{diag} G$ as a $(G \times G)$-variety is identified with $\mathcal{X}(T)$. Similarly, the root monoid $\Gamma \subset \Lambda$ is identified with the root monoid of $\mathcal{X}(T)$, namely with the monoid generated by the positive roots of $G$.

Notice that $\mathrm{k}[G \times G]^{(\operatorname{diag} G)}=\mathrm{k}[G \times G]^{\operatorname{diag} G}=\mathrm{k}[G]$. On the other hand by the Peter-Weyl Theorem we have

$$
\mathrm{k}[G] \simeq \bigoplus_{\lambda \in \mathcal{X}(T)^{+}} \text {End } V(\lambda)
$$

and the multiplication of submodules inside of $\mathrm{k}[G]$ is described in terms of the tensor product as follows:

$$
\text { End } V(\lambda) \text { End } V(\mu)=\bigoplus_{V(\nu) \subset V(\lambda) \otimes V(\nu)} \text { End } V(\nu) \text {. }
$$

Identifying $\Lambda$ and $\mathcal{X}(T)$, it follows that

$$
\Gamma^{+}=\left\{\lambda+\mu-\nu \mid \lambda, \mu, \nu \in \mathcal{X}(T)^{+} \text {and } V(\nu) \subset V(\lambda) \otimes V(\nu)\right\},
$$

namely $\Gamma^{+}$is the monoid generated by the positive roots of $G$. Thanks to the following theorem, this implies that $\mathcal{V}=-\operatorname{cone}\left(\Gamma^{+}\right)^{\vee}$ is the negative Weyl chamber of $\mathcal{X}(T)^{\vee}$.

Theorem 10.5 ([25, Proposition 2.1], [17, Lemma 6.1]). Regarding $\mathcal{V}$ as a subset of $\mathcal{Q}$, it holds

$$
\mathcal{V}=\left\{\nu \in \mathcal{Q} \mid\langle\nu, \gamma\rangle \leqslant 0 \quad \text { for all } \gamma \in \Gamma^{+}\right\}
$$

In particular, $\mathcal{V}$ is a convex cone in $\mathcal{Q}$.

Proof We already noticed that every $\nu \in \mathcal{V}$ satisfies the inequalities $\langle\nu, \gamma\rangle \leqslant 0$, where $\gamma \in \Gamma^{+}$. Suppose conversely that $\nu \in \mathcal{Q}$ satisfies all such inequalities. We proceed as in the proof of Theorem 6.5 , applied to the pair $\left(\mathbb{Q}^{+} \nu, \varnothing\right)$, and show that it is indeed a colored cone.

Let $\lambda_{1}, \ldots, \lambda_{s} \in \Lambda$ be generators of the semigroup $\{\lambda \in \Lambda \mid\langle\nu, \lambda\rangle \geqslant 0\}$ and let $g_{1}, \ldots, g_{s} \in \mathrm{k}(G / H)^{(B)}$ be functions whose weights are respectively $\lambda_{1}, \ldots, \lambda_{s}$. Let $f_{0} \in \mathrm{k}[G]^{(B \times H)}$ be a function vanishing on $\pi^{-1}(D)$ for all $D \in \Delta$ such that $f_{i}:=f_{0} g_{i} \in \mathrm{k}[G]$ for all $i=1, \ldots, s$. Let $V \subset \mathrm{k}[G]^{(H)}$ be the submodule generated by $f_{0}, \ldots, f_{s}$, as in the proof of Theorem 6.5 we have an equivariant morphism $\varphi: G / H \rightarrow \mathbb{P}\left(V^{*}\right)$. Denote $X_{0}^{\prime}=\left\{x \in \overline{\varphi(G / H)} \mid f_{0}(x) \neq 0\right\}$ and $X^{\prime}=G X_{0}^{\prime}$, then by construction we have

$$
\mathrm{k}\left[X_{0}^{\prime}\right]^{(B)} \supset\left\{f^{\prime} \in \mathrm{k}(G / H)^{(B)} \mid \nu\left(f^{\prime}\right) \geqslant 0\right\} .
$$

For $i=0, \ldots, s$, let $M_{i} \subset \mathrm{k}[G]^{(H)}$ be the submodule generated by $f_{i}$. Then every element in $\mathrm{k}\left[X_{0}^{\prime}\right]^{(B)}$ is of the shape $f^{\prime}=f / f_{0}^{N}$, where $N=\sum_{i=0}^{s} n_{i}$ and $f \in\left(M_{0}^{n_{0}} \cdots M_{s}^{n_{s}}\right)^{(B)}$. By the definition of $\Gamma^{+}$, we have

$$
n_{1} \lambda_{1}+\ldots+n_{s} \lambda_{s}-\lambda_{f^{\prime}}=n_{0} \lambda_{f_{0}}+\ldots+n_{s} \lambda_{f_{s}}-\lambda_{f} \in \Gamma^{+}
$$


Therefore $\left\langle\nu, \lambda_{f^{\prime}}\right\rangle \geqslant n_{1}\left\langle\nu, \lambda_{1}\right\rangle+\ldots+n_{s}\left\langle\nu, \lambda_{s}\right\rangle \geqslant 0$, and it follows that

$$
\mathrm{k}\left[X_{0}^{\prime}\right]^{(B)}=\left\{f^{\prime} \in \mathrm{k}(G / H)^{(B)} \mid \nu\left(f^{\prime}\right) \geqslant 0\right\} .
$$

The same argument of Lemma 6.7 implies that $\varphi: G / H \rightarrow X^{\prime}$ has finite fibers. Therefore, reasoning as in the proof of Theorem 6.5 , we get a normal $G$-variety $X$ such that $\varphi$ factors through a spherical embedding $G / H \hookrightarrow X$ and a finite morphism $\psi: X \rightarrow X^{\prime}$, and denoting $X_{0}=\psi^{-1}\left(X_{0}^{\prime}\right)$ we get $\mathrm{k}\left[X_{0}\right]^{(B)}=\mathrm{k}\left[X_{0}^{\prime}\right]^{(B)}$.

Let $x_{0} \in X$ be the base point of the embedding. Since $\varphi(D) \cap X_{0}^{\prime}=\varnothing$ for all $D \in$ $\Delta$, we have $X_{0} \cap G x_{0}=B x_{0}$. On the other hand $\mathrm{k}\left[X_{0}\right]^{(B)} \neq \mathrm{k}(G / H)^{(B)}$, therefore $X \neq G x_{0}$ and there exists a closed $G$-orbit $Y \subset X \backslash G x_{0}$, hence $Y \cap X_{0} \neq \varnothing$. Let $\nu^{\prime} \in \mathcal{V}$ be a valuation with center $Y$, then $\nu^{\prime}$ is nonnegative on $\mathrm{k}\left[X_{0}\right]^{(B)}$, and since it is nonzero it follows that $\nu \in \mathbb{Q}^{+} \nu^{\prime}$.

Consider the antidominant chamber in the vector space generated by the coweights

$$
C=\left\{\nu \in \mathcal{X}(B)_{\mathbb{Q}}^{\vee} \mid\langle\nu, \alpha\rangle \leqslant 0 \text { for all positive root } \alpha\right\} .
$$

The inclusion $\Lambda \subset \mathcal{X}(T)$ induces a surjective map $\mathcal{X}(T)_{\mathbb{Q}}^{\vee} \rightarrow \mathcal{Q}$, and we denote by $C_{G / H}$ the image of $C$ in $\mathcal{Q}$.

We denote by $\operatorname{Lin}(\mathcal{V})$ the linear part of $\mathcal{V}$.

Corollary $10.6([7, \S 3.2],[17$, Corollary 6.3]). $\mathcal{V}$ is a finitely generated convex rational cone in $\mathcal{Q}$ which contains $C_{G / H}$ and whose dual cone is generated by $-\Gamma^{+}$. In particular, $\mathcal{V}$ generates $\mathcal{Q}$ as a vector space, and $\operatorname{Lin}(\mathcal{V})=\Gamma^{\perp}$.

Proof It follows by Theorem 10.5 that $\mathcal{V}$ is a convex rational cone in $\mathcal{Q}$. On the other hand by Proposition 8.10 we can cover $\mathcal{V}$ with finitely many finitely generated convex cones, therefore $\mathcal{V}$ is finitely generated as well. We already noticed that $\Gamma^{+} \subset \mathcal{X}(T)$ is contained in the monoid generated by the positive roots of $G$. It follows that $\langle\nu, \gamma\rangle \leqslant 0$ for all $\nu \in C_{G / H}$ and for all $\gamma \in \Gamma^{+}$, hence $C_{G / H} \subset \mathcal{V}$. Since $C$ generates $\mathcal{X}(B)_{\mathbb{Q}}^{\vee}$ as a vector space, it follows that $\mathcal{V}$ generates $\mathcal{Q}$.

\section{Equivariant Automorphisms of A SPHERICAL HOMOGENEOUS SPACE}

We now turn to the description of the group of the equivariant automorphism $\operatorname{Aut}_{G}(G / H)$ of a spherical homogeneous space $G / H$, and to its connections with the cone of invariant valuations $\mathcal{V}(G / H)$. Notice that $\operatorname{Aut}_{G}(G / H)$ is identified with the quotient $\mathrm{N}_{G}(H) / H$, where $\mathrm{N}_{G}(H)$ acts on $G / H$ by right multiplication: $g . x H=x H g^{-1}=x g^{-1} H$ for $x \in G$ and $g \in \mathrm{N}_{G}(H)$. We will denote $\Lambda(G / H)$, $\mathcal{V}(G / H), \mathcal{Q}(G / H), \Delta(G / H), \rho_{G / H}$ simply by $\Lambda, \mathcal{V}, \mathcal{Q}, \Delta, \rho$.

Theorem 11.1 ([7, §5.2], [17, Theorem 7.1]). The equivariant autmorphism group $\mathrm{N}_{G}(H) / H$ of $G / H$ is an extension of a diagonalizable group by a finite p-group, and $\operatorname{dim} \mathrm{N}_{G}(H) / H=\operatorname{dim} \operatorname{Lin}(\mathcal{V})$. In particular, the connected component of the identity is a central torus in $\mathrm{N}_{G}(H) / H$.

Proof Denote $A=\operatorname{Aut}_{G}(G / H)$ and let $B x_{0} \subset G / H$ be the open $B$-orbit (where $H$ is identified with $x_{0}$ ). Notice that $a \in A$ maps $B x_{0}$ to itself. If $L \subset A^{0}$ is a connected subgroup with preimage $H^{\prime} \subset \mathrm{N}_{G}(H)$, it follows that the colored subspace defined by $H^{\prime}$ has no colors, namely it is of the shape $(\mathcal{C}, \varnothing)$ for some $\mathcal{C} \subset \operatorname{Lin}(\mathcal{V})$.

Notice that a connected linear algebraic group is a torus if and only if all its onedimensional connected subgroups are tori: this is clear if the group is reductive, whereas if it is non-reductive then its unipotent radical always contains a onedimensional subgroup isomorphic to $\mathbb{G}_{a}$ (see [31, Lemma 6.3.4]). Suppose that $L \subset A$ is a connected one-dimensional subgroup with colored subspace $(\mathcal{C}, \varnothing)$. Then $\mathcal{C} \neq 0$, hence there exists $f \in \mathrm{k}(G / H)^{(B)}$ such that $\lambda_{f} \notin \mathcal{C}^{\perp}$. By Proposition 9.2 it follows that $\lambda_{f} \notin \Lambda\left(G / H^{\prime}\right)$ : in particular $f$ is not $H^{\prime}$-invariant, hence it is 
nonconstant on $L=H^{\prime} / H \subset B x_{0}$. It follows that $L$ possesses nontrivial characters, hence it is a torus.

Therefore $A^{0}$ is a torus as well, and $A_{\mathrm{u}}$ is a finite unipotent group. Since $\mathrm{N}_{G}(H) / H \subset \operatorname{Aut}_{B}\left(B x_{0}\right)$, it follows that $\mathrm{N}_{G}(H) / H$ is a subquotient of $B$, hence it is an extension of a diagonalizable group by $A_{\mathrm{u}}$. Since $A^{0}$ is connected and $A_{\mathrm{u}}$ is finite, it follows that $A^{0}$ acts trivially on $A_{\mathrm{u}}$, which is a $p$-group since every unipotent element is a $p$-element. Therefore we are left to show that $\operatorname{dim} \mathrm{N}_{G}(H) / H=$ $\operatorname{dim} \operatorname{Lin}(\mathcal{V})$.

Notice that $\operatorname{dim} \mathrm{N}_{G}(H) / H \leqslant \operatorname{dim} \operatorname{Lin}(\mathcal{V})$. Indeed, if $H^{\prime} / H \subset \mathrm{N}_{G}(H) / H$ is connected of dimension $n$ and if $(\mathcal{C}, \varnothing)$ is the corresponding colored subspace, since $H^{\prime} / H$ is a torus we can find a sequence of subgroups

$$
H=H_{0} \subset H_{1} \subset \ldots \subset H_{n}=H^{\prime}
$$

such that $H_{i} / H_{0}$ is connected of dimension $i$. Correspondingly, we get a strictly increasing sequence of colored subspaces $\left(\mathcal{C}_{i}, \varnothing\right)$, and $\operatorname{since} \mathcal{C} \subset \operatorname{Lin}(\mathcal{V})$ it follows $\operatorname{dim} \mathrm{N}_{G}(H) / H \leqslant \operatorname{dim} \operatorname{Lin}(\mathcal{V})$.

To show the equality $\operatorname{dim} \mathrm{N}_{G}(H) / H=\operatorname{dim} \operatorname{Lin}(\mathcal{V})$ we proceed by induction on the codimension of $H$. Let $\nu \in \operatorname{Lin}(\mathcal{V})$ be a nontrivial element, then by Theorem 9.3 there exists a co-connected inclusion $H \subset H^{\prime}$ with colored subspace is $(\mathbb{Q} \nu, \varnothing)$. Let $f \in \mathrm{k}(G / H)^{(B)}$ be such that $\nu(f) \neq 0$, then $f \notin \mathrm{k}\left(G / H^{\prime}\right)$. Since the corresponding colored subspace has no colors, it follows that $B H^{\prime}=B H$, hence we can define an invertible function on $H^{\prime}$ by composing $f$ with the morphism $H^{\prime} \rightarrow H^{\prime} x_{0} \subset B x_{0}$. This function is a multiple of a character $\chi \in \mathcal{X}\left(H^{\prime}\right)$ (see [21, Proposition 1.2]). Denote $K=\operatorname{ker} \chi$, then $H \subset K$ and $K^{0} \subset H^{\prime}$ is a normal subgroup, hence $H^{\prime \prime}:=$ $H K^{0} \subset H^{\prime}$ is a subgroup containing $H$. On the other hand $H^{\prime \prime} / H \simeq K^{0} / K^{0} \cap H$ is connected, and by Theorem 9.3 it defines a proper colored subspace of $(\mathbb{Q} \nu, \varnothing)$. Hence $H^{\prime \prime}=H$, and it follows $K^{0}=H^{0}$.

Notice that $K$ is a spherical subgroup of $G$, and $\Lambda(G / K) \subset \Lambda$ is a sublattice of finite index by Corollary 8.2. Therefore $\mathcal{Q}=\mathcal{Q}(G / K)$ and $\mathcal{V}=\mathcal{V}(G / K)$. By construction we have $H^{\prime} \subset \mathrm{N}_{G}(K)$. Moreover, since $\mathcal{X}\left(H^{\prime}\right)$ is discrete, the connected group $\mathrm{N}_{G}\left(H^{\prime}\right)^{0}$ acts trivially on $\mathcal{X}\left(H^{\prime}\right)$, hence it fixes $\chi$. Therefore $\mathrm{N}_{G}\left(H^{\prime}\right)^{0} \subset \mathrm{N}_{G}(K)$, and by the first part of the proof together with the induction hypothesis it follows

$$
\begin{aligned}
\operatorname{dim} \operatorname{Lin}(\mathcal{V}) & \geqslant \operatorname{dim} \mathrm{N}_{G}(K) / K \geqslant \operatorname{dim} \mathrm{N}_{G}\left(H^{\prime}\right) / H^{\prime}+1= \\
& =\operatorname{dim} \operatorname{Lin}\left(\mathcal{V}\left(G / H^{\prime}\right)\right)+1=\operatorname{dim} \operatorname{Lin}(\mathcal{V}) .
\end{aligned}
$$

This proves $\operatorname{dim} \mathrm{N}_{G}(K) / K=\operatorname{dim} \operatorname{Lin}(\mathcal{V})$. On the other hand $\mathrm{N}_{G}(K)$ acts on the connected components of $K$, and $H$ is a union of such components. Since it is connected, $\mathrm{N}_{G}(K)^{0}$ acts trivially on the components of $K$, therefore it normalizes $H$. Thus the claim follows thanks to the inequalities

$$
\operatorname{dim} \operatorname{Lin}(\mathcal{V})=\operatorname{dim} \mathrm{N}_{G}(K) / K \leqslant \operatorname{dim} \mathrm{N}_{G}(H) / H \leqslant \operatorname{dim} \operatorname{Lin}(\mathcal{V}) .
$$

The argument in the first paragraph of the proof of Theorem 11.1 shows the following.

Corollary 11.2. Let $G / H$ be a spherical homogeneous space. Let $H \subset H^{\prime}$ be a co-connected inclusion, then $H^{\prime} \subset \mathrm{N}_{G}(H)$ if and only if the corresponding colored subspace has no colors. Moreover, the correspondence of Theorem 9.3 induces a bijection

$$
\left\{H \subset H^{\prime} \subset \mathrm{N}_{G}(H) \mid H^{\prime} / H \text { is connected }\right\} \longleftrightarrow\{\text { subspaces of } \operatorname{Lin}(\mathcal{V})\} .
$$

An important consequence of of Theorem 11.1 concerns the existence of canonical embeddings. 
Corollary 11.3. There exists a simple and complete spherical embedding $\mathrm{G} / \mathrm{H} \hookrightarrow$ $X$ if and only if $\mathrm{N}_{G}(H) / H$ is finite.

Definition 11.4. A homogeneous spherical variety $G / H$ (resp. a spherical subgroup $H \subset G$ ) is called sober if $\mathrm{N}_{G}(H) / H$ is finite. A spherical embedding $G / H \hookrightarrow X$ is called canonical if $X$ is complete, simple and toroidal.

Notice that a canonical embedding for $G / H$ exists if and only if $G / H$ is sober, if and only if $\mathcal{V}$ is a strictly convex cone, in which case its colored cone is $(\mathcal{V}, \varnothing)$. In particular, if it exists the canonical embedding of $G / H$ is uniquely determined up to equivariant automorphisms by Theorem 6.5. By Theorem 8.5, the canonical embedding dominates every other simple and complete embedding of $G / H$, and it is dominated by all toroidal embeddings of $G / H$.

Corollary 11.5. The homogeneous space $G / \mathrm{N}_{G}(H)$ is sober.

Proof Denote $H^{\prime}=\mathrm{N}_{G}(H)^{0} H$. Then $H^{\prime}$ is a spherical subgroup of $G$ containing $H$, and $H^{\prime} / H$ is connected. Moreover $\operatorname{dim} H^{\prime} / H=\operatorname{dim} \mathrm{N}_{G}(H) / H$, therefore $H^{\prime} / H$ is the identity component of $\mathrm{N}_{G}(H) / H$ and it follows as in the proof of Theorem 11.1 that the colored subspace of $H^{\prime}$ is $(\operatorname{Lin}(\mathcal{V}), \varnothing)$. Therefore $\mathcal{V}\left(G / \mathrm{N}_{G}(H)\right)=\mathcal{V} / \operatorname{Lin}(\mathcal{V})$ is strictly convex and $\mathrm{N}_{G}(H)$ is sober.

We now consider the other extremal case, namely when $\mathcal{V}=\mathcal{Q}$.

Definition 11.6. The variety $G / H$ is called horospherical if $H$ contains a maximal unipotent subgroup of $G$.

Corollary 11.7 ([17, Corollary 7.2]). The spherical variety $G / H$ is horospherical if and only if $\mathcal{V}=\mathcal{Q}$.

Proof Consider first the case $H=U$. In this case $\Lambda=\mathcal{X}(T)$, and since $\mathrm{N}_{G}(U)=$ $B$ by Theorem 11.1 it follows $\operatorname{dim} \operatorname{Lin}(\mathcal{V})=\operatorname{rk} G=\operatorname{rk} \Lambda$. Therefore $\mathcal{V}=\mathcal{Q}$. Suppose now that $U \subset H$, then we have a surjective equivariant morphism $G / U \rightarrow G / H$ which induces a surjective map $\mathcal{V}(G / U) \rightarrow \mathcal{V}$. Since $\mathcal{V}(G / U)=\mathcal{Q}(G / U)$, it follows that $\mathcal{V}=\mathcal{Q}$.

Suppose now that $\mathcal{V}=\mathcal{Q}$. Let $P \subset \mathrm{N}_{G}(H)$ be the preimage of the identity component of $\mathrm{N}_{G}(H) / H$. By Corollary 11.2, $P$ corresponds to the colored subspace $(\mathcal{Q}, \varnothing)$. Therefore $\operatorname{rk}(G / P)=0$ and by the completeness criterion it follows that $P$ is a parabolic subgroup of $G$. On the other hand $P / H$ is a torus by Theorem 11.1, therefore $H$ contains a maximal unipotent subgroup of $G$.

11.1. Lifting of equivariant automorphisms. Consider the right action of $\mathrm{N}_{G}(H)$ on $G / H$ defined by $g . x H=x g^{-1} H$, where $x \in G$ and $g \in \mathrm{N}_{G}(H)$. For $g \in \mathrm{N}_{G}(H)$, let $\varphi_{g} \in \operatorname{Aut}_{G}(X)$ be the corresponding automorphism. This induces an isomor-

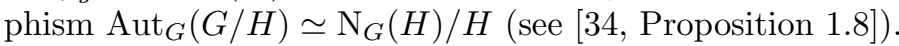

Remark 11.8. Notice that the action of $\mathrm{N}_{G}(H)$ on $G / H$ induces an action of $\mathrm{N}_{G}(H)$ on the set of colors $\Delta(G / H)$. Similarly, we have an induced action of $\mathrm{N}_{G}(H)$ on the weight lattice $\Lambda(G / H)$, which is trivial: if indeed $f \in \mathrm{k}(G / H)^{(B)}$ has weight $\lambda$, then $g . f \in \mathrm{k}(G / H)^{(B)}$ has also weight $\lambda$. It follows that $\mathrm{N}_{G}(H)$ preserves the fibers of the map $\rho: \Delta(G / H) \rightarrow \mathcal{Q}(G / H)$. In particular, if $\rho$ is injective, then $\mathrm{N}_{G}(H)$ acts trivially on $\Delta(G / H)$ as well.

Definition 11.9. Let $X$ be a spherical $G$-variety. A color $D \in \Delta(X)$ is called undetermined if the fiber $\rho_{X}^{-1}\left(\rho_{X}(D)\right)$ has cardinality greater than one.

The basic example of undetermined colors comes from $\mathrm{SL}_{2} / T$ : in that case we have $\Delta=\left\{D^{+}, D^{-}\right\}$, and $\rho\left(D^{+}\right)=\rho\left(D^{-}\right)$(see Example 3.3).

Applying Theorem 8.5 to $\varphi_{g} \in \operatorname{Aut}_{G}(G / H)$ we get the following description. 
Corollary 11.10. Let $G / H \hookrightarrow X$ be a spherical embedding. Then

$\operatorname{Aut}_{G}(X)=\left\{\varphi_{g} \in \operatorname{Aut}_{G}(G / H) \mid g \cdot \Delta_{Y}(X)=\Delta_{Y}(X)\right.$ for every closed $G$-orbit $\left.Y \subset X\right\}$

In particular, if no undetermined color of $X$ contains a $G$-orbit, then $\operatorname{Aut}_{G}(X) \simeq$ $\mathrm{N}_{G}(H) / H$.

Proof Let $g \in \mathrm{N}_{G}(H)$. By Remark 11.8, it follows that $\left(\varphi_{g}\right)_{*}: \Lambda(G / H) \rightarrow$ $\Lambda(G / H)$ is the identity. Therefore, by Theorem $8.5, \varphi_{g}$ extends to an equivariant automorphism of $X$ if and only if $g \cdot \Delta_{Y}(X)=\Delta_{Y}(X)$ for every $G$-orbit $Y \subset X$, and the claim follows by Remark 8.4.

We now introduce a remarkable subgroup of $\mathrm{N}_{G}(H)$ containing $H$.

Definition 11.11. The spherical closure of $H$, denoted by $\bar{H}$, is the kernel of the action of $\mathrm{N}_{G}(H)$ on $\mathcal{X}(H)$. If $H=\bar{H}$, then $H$ is called spherically closed.

Notice that $\bar{H}$ contains the center of $G$, and it has finite index in $\mathrm{N}_{G}(H)$ : indeed $\mathcal{X}(H)$ is discrete, therefore $\mathrm{N}_{G}(H)^{0}$ acts trivially on $\mathcal{X}(H)$.

Proposition 11.12. The spherical closure $\bar{H}$ is the kernel of the action of $\mathrm{N}_{G}(H)$ on $\Delta(G / H)$. In particular, if $\rho: \Delta(G / H) \rightarrow \mathcal{Q}(G / H)$ is injective, then $\bar{H}=$ $\mathrm{N}_{G}(H)$.

Proof Let $p: G_{0} \rightarrow G$ be a finite cover such that $\operatorname{Pic}\left(G_{0}\right)=0$ (see [20, Proposition 4.6]). Let $\Gamma \subset Z\left(G_{0}\right)$ be the kernel of $p$ and set $H_{0}=p^{-1}(H)$. Notice that $\mathrm{N}_{G}(H)=\mathrm{N}_{G_{0}}\left(H_{0}\right) / \Gamma$ and $\bar{H}=\bar{H}_{0} / \Gamma$. Thus we can assume that $\operatorname{Pic}(G)=0$, namely that $\mathrm{k}[G]$ is a factorial ring (see [12, Proposition B.72]).

Let $\pi: G \rightarrow G / H$ be the projection. For $D \in \Delta(G / H)$, let $f_{D} \in \mathrm{k}[G]^{(B \times H)}$ be a defining function for the divisor $\pi^{-1}(D)$. By [21, Proposition 1.2] every function $f \in \mathrm{k}[G]$ which is nowhere vanishing is a multiple of a character of $G$. On the other hand by Proposition 2.20 the irreducible components $G \backslash B H$ are precisely the divisors $\pi^{-1}(D) \subset G$, thus up to a scalar factor every $f \in \mathrm{k}(G)^{(B \times H)}$ is the product of a Laurent monomial in the functions $f_{D}$ and a character of $G$.

Since $B H$ is open in $G$, notice that

$$
\mathrm{k}(G)^{(B \times H)}=\mathrm{k}[B H]^{(B \times H)}=\mathrm{k}[(B \times H) / \operatorname{diag}(B \cap H)]^{(B \times H)} .
$$

Therefore the set of weights occurring in $\mathrm{k}(G)^{(B \times H)}$ is $\mathcal{X}(B) \times \mathcal{X}(B \cap H) \mathcal{X}(H)$. If $\left(\omega_{D}, \chi_{D}\right) \in \mathcal{X}(B) \times \mathcal{X}(H)$ is the weight of $f_{D}$, it follows that $\mathcal{X}(B) \times \mathcal{X}(B \cap H) \mathcal{X}(H)$ is generated by the weights $\left(\omega_{D}, \chi_{D}\right)$ together with the image of the restriction $\mathcal{X}(G) \rightarrow \mathcal{X}(B) \times \mathcal{X}(H)$. On the other hand the restriction $\mathcal{X}(B) \rightarrow \mathcal{X}(B \cap H)$ is surjective (see [1, Proposition 8.2]), therefore the projection

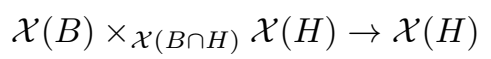

is surjective as well, and $\mathcal{X}(H)$ is generated by the characters $\chi_{D}$ with $D \in \Delta(G / H)$ together with the image of the restriction $\mathcal{X}(G) \rightarrow \mathcal{X}(H)$.

Since $\mathrm{N}_{G}(H)$ acts trivially on the image of $\mathcal{X}(G) \rightarrow \mathcal{X}(H)$, it follows that an element $g \in \mathrm{N}_{G}(H)$ acts trivially on $\mathcal{X}(H)$ if and only if it acts trivially on the characters $\chi_{D}$ with $D \in \Delta(G / H)$. Consider now the action of $\mathrm{N}_{G}(H)$ on $\Delta(G / H)$, and notice that

$$
\left(\omega_{g . D}, \chi_{g . D}\right)=\left(\omega_{D}, g \cdot \chi_{D}\right) .
$$

Thus $g \cdot D=D$ if and only if $g \cdot \chi_{D}=\chi_{D}$, and $g$ acts trivially on $\Delta(G / H)$ if and only if it acts trivially on $\mathcal{X}(H)$.

The last claim follows by Remark 11.8.

Corollary 11.13. Let $G / H \hookrightarrow X$ be a spherical embedding, then $\bar{H} / H \subset \operatorname{Aut}_{G}(X)$. 
Suppose now that $X$ is an affine spherical variety. Since $X$ has an open $G$-orbit, it follows that $\mathrm{k}[X]^{G}=\mathrm{k}$ : therefore $X / / G$ is a single point and $X$ is a simple spherical variety. More precisely, affine spherical varieties can be characterized as follows.

Theorem 11.14 ([17, Theorem 6.7]). Let $X$ be a simple spherical variety, with closed $G$-orbit $Y$. Then $X$ is affine if and only if there exists $\lambda \in \Lambda(X)$ such that:

ii) $\lambda \in \mathcal{C}_{Y}(X)^{\perp}$;

i) $\langle\nu, \lambda\rangle \leqslant 0$ for all $\nu \in \mathcal{V}(X)$;

ii) $\langle\rho(D), \lambda\rangle>0$ for all $D \in \Delta(X) \backslash \Delta_{Y}(X)$.

In particular we get the following corollary, which holds in much greater generality (see [18, Lemma 6.6] and its remark).

Corollary 11.15. Let $G / H \hookrightarrow X$ be a spherical embedding and assume that $X$ is quasi-affine, then $\operatorname{Aut}_{G}(X)=\operatorname{Aut}_{G}(G / H)$.

Proof Suppose that $X$ is affine, with closed $G$-orbit $Y$. By Remark 8.4 together with Corollary 11.10 it is enough to check that $g \cdot \Delta_{Y}(X)=\Delta_{Y}(X)$ for all $g \in$ $\mathrm{N}_{G}(H)$. By the previous theorem we have $\langle\rho(D), \lambda\rangle=0$ for all $D \in \Delta_{Y}(X)$, and $\langle\rho(D), \lambda\rangle>0$ for all $D \in \Delta(X) \backslash \Delta_{Y}(X)$. It follows that $\rho^{-1}(\rho(D)) \subset \Delta_{Y}(X)$ for all $D \in \Delta_{Y}(X)$, therefore $\operatorname{Aut}_{G}(X)=\operatorname{Aut}_{G}(G / H)$ by Remark 11.8.

Suppose now that $X$ is quasi-affine. The claim follows if we show that there exists an equivariant open embedding $X \hookrightarrow Z$ for some normal affine $G$-variety $Z$. Suppose that $\iota^{\prime}: X \hookrightarrow Z^{\prime}$ is an open embedding of $X$ inside an affine variety $Z^{\prime}$, and denote $A^{\prime}=\mathrm{k}\left[Z^{\prime}\right]$. Then $A^{\prime}$ identifies with a finitely generated subalgebra of $\mathrm{k}[X]$, and by Lemma 2.3 there exists a $G$-stable finitely generated subalgebra $A \subset \mathrm{k}[X]$ containing $A^{\prime}$. Since $X$ is normal, we may also assume that $A$ is integrally closed. Therefore $Z=\operatorname{Spec}(A)$ is a normal affine $G$-variety with an equivariant dominant morphism $\iota: X \rightarrow Z$. Since $\iota^{\prime}$ factors through $\iota$, the latter is an open embedding as well.

\section{WONDERFUL EMBEDDINGS AND SPHERICAL ROOTS}

We now report without proof a deep theorem concerning the structure of the valuation cone of a spherical homogeneous space, due to Brion [4] when $\operatorname{char}(\mathrm{k})=0$ and to Knop [19] in arbitrary characteristic. We keep the notation of the previous section: $G / H$ is a spherical homogeneous space, and we denote $\Lambda(G / H), \mathcal{V}(G / H)$, $\mathcal{Q}(G / H)$ simply by $\Lambda, \mathcal{V}, \mathcal{Q}$.

Let $W_{G / H} \subset \mathrm{GL}(\mathcal{Q})$ be the group generated by the reflections about the codimension one faces of $\mathcal{V}$.

Theorem 12.1 ([4, Théorème 3.5], [19, Theorem 4.6]). $W_{G / H}$ is a finite reflection group. If moreover $\operatorname{char}(\mathrm{k}) \neq 2$, then $\mathcal{V}$ is a fundamental domain for its action on $\mathcal{Q}$.

Remark 12.2. The last claim of the previous theorem is false if $\operatorname{char}(\mathrm{k})=2$ : in that case $\mathcal{V}$ is only a union of Weyl chambers for $W_{G / H}$. For example, if $G / H=$ $\mathrm{PGL}_{2} / \mathrm{SO}_{3}$, then $\mathcal{V}$ is the union of two Weyl chambers (see [30] and [19, Remark 4.4]).

Let $\Sigma_{G / H}$ be the set of the primitive elements in $\Lambda$ which generate an extremal ray of $-\mathcal{V}^{\vee}$. In particular, we get the following corollary.

Corollary 12.3. Suppose that $\operatorname{char}(\mathrm{k}) \neq 2$. Then $\mathcal{V}$ is a cosimplicial cone, and $\Sigma_{G / H}$ is the basis of a root system with Weyl group $W_{G / H}$. 
Definition 12.4. The elements of $\Sigma_{G / H}$ are called the spherical roots of $G / H$, and $W_{G / H}$ is called the little Weyl group of $G / H$.

To unburden the notation, from now on we will denote $\Sigma_{G / H}$ simply by $\Sigma$.

Remark 12.5. Let $\Gamma^{+}$be the root monoid of $G / H$ introduced in Definition 10.1. Notice that by definition we have cone $\left(\Gamma^{+}\right)=-\mathcal{V}^{\vee}=\operatorname{cone}(\Sigma)$. If $\operatorname{char}(\mathrm{k})=0$ and $H$ is spherically closed, then $\Gamma^{+}=\mathbb{N} \Sigma$ (see Corollary 12.14 and the discussion preceding it). However, as illustrated by the following examples, in general it might be $\Sigma \not \subset \Gamma^{+}$: for instance, by Remark 10.2, this happens whenever $\Sigma$ is not contained in the sublattice of $\mathcal{X}(T)$ generated by the roots of $G$.

Example 12.6. Assume that $\operatorname{char}(\mathrm{k})=0$. Let $G=\mathrm{SL}_{2} \times \mathrm{SL}_{2}$ and $H=\operatorname{diag}\left(\mathrm{SL}_{2}\right)$ : then by Examples 3.6 and 10.4 we have identifications $\Lambda=\mathbb{Z} \omega$ and $\Gamma^{+}=\mathbb{N} \alpha$ (where $\omega$ is the fundamental weight of $\mathrm{SL}_{2}$ and $\alpha=2 \omega$ is the corresponding simple root), thus $\Sigma=\{\omega\}$ is not contained in $\Gamma^{+}$. Suppose now $G=\mathrm{SO}_{2 n+1}$ and $H=\mathrm{SO}_{2 n}$ with $n \geqslant 2$ : then $G / H$ is spherical, and if $\alpha_{1}, \ldots, \alpha_{n}$ denote the simple roots of $\mathrm{SO}_{2 n+1}$ we have the equalities $\Sigma=\left\{\alpha_{1}+\ldots+\alpha_{n}\right\}$ and $\Gamma^{+}=2 \mathbb{N}\left(\alpha_{1}+\ldots+\alpha_{n}\right) . \quad \triangle$

From now on we will assume that char $(\mathrm{k})=0$.

In this case there is a much more explicit description of the automorphism group $\mathrm{N}_{G}(H) / H$. Denote by $\Lambda_{\mathrm{N}}$ the weight lattice of $G / \mathrm{N}_{G}(H)$. The following is an immediate corollary of Theorem 11.1.

Corollary 12.7. The automorphism group $\mathrm{N}_{G}(H) / H$ is diagonalizable, and

$$
\mathrm{N}_{G}(H) / H \simeq \operatorname{Hom}_{\mathbb{Z}}\left(\Lambda / \Lambda_{\mathrm{N}}, \mathbb{G}_{m}\right) .
$$

Proof The first claim follows by Theorem 11.1, and the second one follows by noticing that $\mathcal{X}\left(\mathrm{N}_{G}(H) / H\right)=\Lambda / \Lambda_{\mathrm{N}}$ thanks to Proposition 8.1.

Definition 12.8. If the canonical embedding $G / H \hookrightarrow X$ exists and is smooth, then it is called a wonderful embedding, and $H$ is called a wonderful subgroup of $G$.

Suppose that $G / H \hookrightarrow X$ is the wonderful embedding of a spherical homogeneous space. Then $X$ has several remarkable properties:

i) $X$ is smooth and projective, with an open orbit $G x_{0}$;

ii) Let $X \backslash G x_{0}=\bigcup_{i=1}^{r} X_{i}$ be the decomposition into irreducible components, then every $X_{i}$ is a $G$-stable smooth prime divisor;

iii) The divisors $X_{1}, \ldots, X_{r}$ intersect transversally, and their common intersection is a single $G$-orbit;

iv) For all $x \in X \backslash G x_{0}$, we have the equality $\overline{G x}=\cap_{x \in X_{i}} X_{i}$.

A $G$-variety satisfying the previous properties is called wonderful. Every wonderful $G$-variety is spherical (see [23]), and a spherical embedding $G / H \hookrightarrow X$ is wonderful if and only if $X$ is a wonderful $G$-variety (see e.g. [27, 3.3.1]). For more on wonderful varieties, we refer to [27] and [28].

Proposition 12.9. The homogeneous space $G / H$ admits a wonderful embedding if and only if $\Sigma$ is a basis of $\Lambda$.

Proof By Corollary 11.3, $G / H$ admits a canonical embedding if and only if $\mathcal{V}$ is a strictly convex cone. On the other hand, by the local structure theorem for toroidal varieties (see [26, Theorem 3.4.1]), the canonical compactification of $G / H$ is smooth if and only if $\Sigma$ is a basis for $\Lambda$.

Theorem 12.10 ([18, Corollaries 6.5 and 7.2$])$. The canonical embedding of $G / \mathrm{N}_{G}(H)$ is smooth. In particular, $G / \mathrm{N}_{G}(H)$ admits a wonderful embedding.

Equivalently, by Proposition 12.9, the previous theorem can be stated as follows: let $\Sigma_{\mathrm{N}} \subset \Lambda_{\mathrm{N}}$ be the set of spherical roots of $G / \mathrm{N}_{G}(H)$, then $\Lambda_{\mathrm{N}}=\mathbb{Z} \Sigma_{\mathrm{N}}$. 
Remark 12.11. The set $\Sigma_{\mathrm{N}}$ was explicity described by Losev [22, Theorem 2]. His description implies that $\Sigma_{\mathrm{N}} \subset \Sigma \cup 2 \Sigma$. In particular, if $H$ is a wonderful subgroup of $G$, by Corollary 12.7 we get that $\mathrm{N}_{G}(H) / H \simeq(\mathbb{Z} / 2 \mathbb{Z})^{m}$ for some $m \leqslant \operatorname{rk}(G / H)$. For example, if $G=\mathrm{SL}_{2}$ and $H=T$, as we have seen in Examples 8.11 and 8.12 we have $\Sigma=\{\alpha\}$ and $\Sigma_{\mathrm{N}}=\{2 \alpha\}$, and $\mathrm{N}_{\mathrm{SL}_{2}}(T) / T \simeq \mathbb{Z} / 2 \mathbb{Z}$.

The set of spherical roots $\Sigma_{\mathrm{N}}$ arises naturally when considering the multiplication of regular functions on a quasi-affine spherical homogeneous space $G / H$ (e.g., when $H$ is reductive), in a very similar manner as we introduced the root monoid $\Gamma^{+}$in Definition 10.1. Indeed, keeping the notation therein, we can consider the multiplication of $B$-eigenfunctions in $\mathrm{k}[G]^{H}=\mathrm{k}[G / H]$ instead of $\mathrm{k}[G]^{(H)}$ and define a sublattice $\Gamma_{\mathrm{N}} \subset \Gamma$ as the lattice generated by the elements $\gamma\left(f_{1}, f_{2}, f\right)$ such that $f_{1}, f_{2}, f \in \mathrm{k}[G / H]^{(B)}$, and a monoid $\Gamma_{\mathrm{N}}^{+} \subset \Gamma^{+}$by setting $\Gamma_{\mathrm{N}}^{+}=\Gamma_{\mathrm{N}} \cap \operatorname{cone}\left(\Gamma^{+}\right)$.

Theorem 12.12 ([18, Theorem 1.3]). We have equalities $\Gamma_{\mathrm{N}}=\mathbb{Z}_{\Sigma_{\mathrm{N}}}$ and $\Gamma_{\mathrm{N}}^{+}=$ $\mathbb{N} \Sigma_{\mathrm{N}}$.

Consider now the spherical closure $\bar{H}$ introduced in Definition 11.11. Notice that $\bar{H}$ is a sober spherical subgroup of $G$ : indeed it has finite index in $\mathrm{N}_{G}(H)$, therefore $\mathcal{V}(G / \bar{H})=\mathcal{V}\left(G / \mathrm{N}_{G}(H)\right)$, hence $\bar{H}$ is sober by Corollary 11.5. For more properties of the spherical closure, see [3, Section 2.4].

Theorem 12.13 ([18, Theorem 7.5 and Corollary 7.6]). The canonical embedding of $G / \bar{H}$ is smooth. In particular, $G / \bar{H}$ admits a wonderful embedding.

Denote by $\Lambda_{\mathrm{sc}} \subset \Lambda$ the weight lattice of $G / \bar{H}$, and by $\Sigma_{\mathrm{sc}} \subset \Lambda_{\mathrm{sc}}$ the set of spherical roots of $G / \bar{H}$. Then by Proposition 12.9 the previous theorem is equivalent to the equality $\Lambda_{\mathrm{sc}}=\mathbb{Z} \Sigma_{\mathrm{sc}}$. Notice that, by the definition of $\bar{H}$, we have

$$
\mathrm{k}[G]^{(B \times H)}=\mathrm{k}[G]^{(B \times \bar{H})} .
$$

Therefore $G / H$ and $G / \bar{H}$ have the same root monoid $\Gamma^{+}$, and by Remark 12.5 we get $\Gamma^{+} \subset \Lambda_{\mathrm{sc}} \cap \operatorname{cone}\left(\mathbb{N} \Sigma_{\mathrm{sc}}\right)=\mathbb{N} \Sigma_{\mathrm{sc}}$. On the other hand it is possible to check that $\Sigma_{\mathrm{sc}} \subset \Gamma^{+}$(see e.g. [2, Proposition 5]), so that $\Gamma^{+}=\mathbb{N} \Sigma_{\mathrm{sc}}$ and $\Gamma=\Lambda_{\mathrm{sc}}$ : that is, the root lattice of $G / H$ is the weight lattice of $G / \bar{H}$. Reasoning as in Corollary 12.7, we get then the following description.

Corollary 12.14. We have an isomorphism $\bar{H} / H \simeq \operatorname{Hom}_{\mathbb{Z}}\left(\Lambda / \Gamma, \mathbb{G}_{m}\right)$.

\section{REFERENCES}

[1] Borel, A.: Linear algebraic groups, 2nd ed., Grad. Texts in Math., 126, Springer-Verlag, New York, 1991

[2] Bravi, P., Gandini, J., Maffei, A.: Projective normality of model varieties and related results, Represent. Theory, 20, 39-93 (2016)

[3] Bravi, P., Luna, D.: An introduction to wonderful varieties with many examples of type $\mathrm{F}_{4}$, J. Algebra, 329, 4-51 (2011)

[4] Brion, M.: Vers une généralization des espaces symétriques, J. Algebra, 134, 115-143 (1990)

[5] Brion, M.: Variétés sphériques, http://www-fourier.ujf-grenoble.fr/ mbrion

[6] Brion, M.: Introduction to actions of algebraic groups, Les cours du C.I.R.M., 1, 1-22, (2010)

[7] Brion, M., Pauer, F.: Valuations des espaces homogènes sphériques, Comment. Math. Helv., 62, 265-285, (1987)

[8] Donkin, S.: The normality of closures of conjugacy classes of matrices, Invent. Math., 101, $717-736,(1990)$

[9] Ferrer-Santos, W., Rittatore, A.: Actions and Invariants of Algebraic Groups, Chapman \& Hall/CRC, Boca Raton, 2005

[10] Fulton, W.: Introduction to toric varieties, Princeton University Press, Princeton, 1993

[11] Goodman, R., Wallach, N.R.: Symmetry, Representations, and Invariants, Grad. Texts in Math., 255, Springer, Dordrecht, 2009

[12] Görz, U., Wedhorn, T.: Algebraic Geometry I, Vieweg+Teubner Verlag, Wiesbaden, 2010 
[13] Grosshans, F.: Algebraic Homogeneous Spaces and Invariant Theory, Lecture Notes in Math., 1673, Springer-Verlag, Berlin, 1997

[14] Hartshorne, R.: Ample subvarieties of algebraic varieties, Lecture Notes in Math., 156, Springer-Verlag, Berlin-Heidelberg-New York, 1970

[15] Jantzen, J.C.: Representations of algebraic groups, 2nd ed., Math. Surveys Monogr., 107, American Mathematical Society, Providence, RI, 2003

[16] Kempf, G., Knudsen, F., Mumford, D., Saint-Donat, B.: Toroidal Embeddings I, Lecture Notes in Math., 339, Springer, Berlin-Heidelberg, 1973

[17] Knop, F.: The Luna-Vust Theory of spherical embeddings, in: Proceedings of the Hyderabad Conference on Algebraic Groups (Hyderabad, 1989), 225-249, Manoj Prakashan, Madras, 1991

[18] Knop, F.: Automorphisms, root systems, and compactifications of homogeneous varieties, $J$. Amer. Math. Soc., 9, 153-174, (1996)

[19] Knop, F.: Spherical roots of spherical varieties, Ann. Inst. Fourier (Grenoble), 64, 25032526, (2014)

[20] Knop, F., Kraft, H., Luna, D., Vust, Th.: Local properties of algebraic group actions, in: Algebraische Transformationsgruppen und Invariantentheorie (H. Kraft, P. Slodowy, T.A. Springer eds.), 63-76, DMV Semin., 13, Birkhäuser, Basel-Boston-Berlin, 1989

[21] Knop, F., Kraft, H., Vust, Th.: The Picard group of a $G$-variety, in: Algebraische Transformationsgruppen und Invariantentheorie (H. Kraft, P. Slodowy, T.A. Springer eds.), 77-88, DMV Semin., 13, Birkhäuser, Basel-Boston-Berlin, 1989

[22] Losev, I.: Uniqueness property for spherical homogeneous spaces, Duke Math. J., 147, 315$343,(2009)$

[23] Luna, D.: Toute variété magnifique est sphérique, Transform. Groups, 1, 249-258, (1996)

[24] Luna, D., Vust, Th.: Plongements d'espaces homogènes, Comment. Math. Helv., 58, 186-245, (1983)

[25] Pauer, F.: Caractérisation valuative d'une classe de sous-groupes dun groupe algébrique, $C$. R. 109 $^{\mathrm{e}}$ Congrès nat. Soc. sav., 3 , 159-166 (1984)

[26] Perrin, N.: Sanya lectures: Geometry of spherical varieties, to appear in Acta Math. Sin. (Engl. Ser.)

[27] Pezzini, G.: Lectures on spherical and wonderful varieties, Les cours du C.I.R.M., 1, 33-53 (2010)

[28] Pezzini, G.: Lectures on wonderful varieties, submitted to Acta Math. Sin. (Engl. Ser.)

[29] Popov, V.L., Vinberg, E.B.: Invariant theory, in: Algebraic Geometry IV, Encyclopaedia Math. Sci., 55, 123-284, Springer, Berlin-Heidelberg-New York, 1994

[30] Schalke, B.: Sphärische Einbettungen in positiver Charakteristik, Diplomarbeit (Universität Erlangen), 2011

[31] Springer, T.A.: Linear algebraic groups, 2nd ed., Progr. Math., 9, Birkhäuser, Boston, MA, 1998

[32] Sumihiro, H.: Equivariant completion, J. Math. Kyoto Univ., 14, 1-28, (1974)

[33] Timashev, D.A.: Equivariant compactifications of reductive groups, Sb. Math., 194, 589-616, (2003)

[34] Timashev, D.A.: Homogeneous spaces and equivariant embeddings, Encyclopaedia Math. Sci., 138, Springer, Berlin-Heidelberg, 2011

[35] Vust, Th.: Plongements d'espaces symétriques algébriques: une classification, Ann. Sc. Norm. Super. Pisa Cl. Sci. (4), 17, 165-195, (1990)

Current address: Scuola Normale Superiore, Piazza dei Cavalieri 7, 56126 Pisa, Italy

E-mail address: jacopo.gandini@sns.it 